\title{
THE LOGARITHMIC SOBOLEV CONSTANT OF KAWASAKI DYNAMICS UNDER A MIXING CONDITION REVISITED
}

\author{
N. CANCRINI ${ }^{\mathrm{a}}$, F. MARTINELLI ${ }^{\mathrm{b}}$, C. ROBERTO $^{\mathrm{c}}$ \\ a Dipartimento di Energetica, Università dell'Aquila, Italy and INFM Unità di Roma "La Sapienza", \\ Roma, Italy \\ ${ }^{\mathrm{b}}$ Dipartimento di Matematica, Università di Roma Tre, Roma, Italy \\ ${ }^{\mathrm{c}}$ Département de Mathématiques, Laboratoire de Statistique et Probabilités, Université Paul-Sabatier, \\ Toulouse, France
}

Received 20 September 2000, revised 2 April 2001

ABSTRACT. - We consider a conservative stochastic spin exchange dynamics reversible with respect to the canonical Gibbs measure of a lattice gas model. We assume that the corresponding grand canonical measure satisfies a suitable strong mixing condition. Following previous work by two of us for the spectral gap, we provide an alternative and quite natural, from the physical point of view, proof of the well known result of Yau stating that the logarithmic Sobolev constant in a box of side $L$ grows like $L^{2}$. ๑ 2002 Éditions scientifiques et médicales Elsevier SAS

AMS classification: 82B44; 82C22; 82C44; 60K35

Keywords: Kawasaki dynamics; Logarithmic Sobolev constant; Equivalence of ensembles; Concentration inequalities

RÉSUMÉ. - Soit une mesure de Gibbs canonique d'un modèle de gaz sur un réseau. Nous lui associons des dynamiques conservatives d'échange de spins pour les quelles elle est reversible. Nous supposons que la mesure de Gibbs grand canonique correspondante vérifie une condition de mélange appropriée. En s'appuyant sur les travaux antérieurs de deux d'entre nous sur le trou spectral nous donnons une preuve alternative et assez naturelle, du point de vue de la physique, du fameux résultat de Yau qui stipule que la constante de Sobolev logarithmique dans une boîte de taille $L$ croît comme $L^{2}$. ๑ 2002 Éditions scientifiques et médicales Elsevier SAS

Mots Clés: Dynamiques de Kawasaki; Constante de Sobolev logarithmique; Equivalence des ensembles; Inégalités de concentration

E-mail addresses: nicoletta.cancrini@ romal.infn.it (N. Cancrini), martin@mat.uniroma3.it (F. Martinelli), roberto@ cict.fr (C. Roberto). 


\section{Introduction}

In this paper we provide a new, independent proof of the famous result of Yau [19] which states that the logarithmic Sobolev constant of a spin exchange dynamics in a box of side $L$ of $\mathbb{Z}^{d}$, reversible w.r.t. the canonical Gibbs measure of a finite range lattice gas, grows like $L^{2}$, provided that the corresponding grand canonical Gibbs measure satisfies a suitable "high temperature" condition. We thus complete the program that was begun in [10] were a similar scaling law was proved for the inverse spectral gap.

The problem of computing the relaxation time of stochastic Monte Carlo algorithms for models of classical spin systems in $\mathbb{Z}^{d}$ has attracted in the last years considerable attention and many new rigorous techniques have been developed giving rise to nice progresses in probability theory and statistical mechanics. If, for simplicity, we confine ourselves to \pm 1 (or $0-1$ in the lattice gas picture) spins, the two most studied random dynamics have been non-conservative Glauber type algorithms, in which a spin at a time flips its value with a rate satisfying the detailed balance condition w.r.t. the grand canonical Gibbs measure, and conservative Kawasaki dynamics in which nearest neighbors spins exchange their values with a rate satisfying the detailed balance condition w.r.t. the canonical Gibbs measure.

For Glauber dynamics the general picture is relatively clear for a wide class of models both in the one phase and in the phase coexistence region with the notable exception of the critical point (see e.g. [16] and references therein).

For Kawasaki dynamics, instead, the presence of a conservation law makes the analysis of the relaxation properties much more difficult than in the non-conservative case, with interesting analogies with the problem of the Goldstone mode in quantum mechanics [1], and many interesting questions are still open both in the one phase region as well as in the presence of phase coexistence (see [7]).

One important class of results for Kawasaki dynamics concerns the relaxation behaviour under a suitable "high temperature condition". Such a condition (see Section 2.4 below for a precise description) requires in particular the exponential decay of the grand canonical covariances uniformly in the chemical potential, i.e. in the particle density, and therefore it cannot be true at low temperature where, in general, phase coexistence takes place for certain values of the density.

Under the validity of such a condition, the most important result is the so called diffusive scaling $L^{2}$ for the relaxation time (in what follows identified with the inverse of the spectral gap of the generator) of the Kawasaki dynamics in a box of side $L$, first proved in [14] and then, much later and by different methods, in [10] (see also [11] for an extension to a spin system with random interactions). Such a result is a key stone in the study of the hydrodynamical limit of the Ising model [18] and it plays an essential role in the proof of the power law $L^{2}$-decay to equilibrium of local observables (see [10]). Quite interesting its proof poses new, non-trivial problems in the theory of canonical Gibbs measures, their large deviations properties and their detailed equivalence to grand canonical ones (see $[5,6,9,4]$ ). Some time after the basic paper [14], the above result was considerably strengthen in [19] in that the same diffusive scaling was proved also for the logarithmic Sobolev constant (see also $[15,20]$ for related work). 
The basic strategy of [19] is the martingale approach developed in [14] together with entropy bounds, large deviations estimate for canonical Gibbs measure and partial averaging on mesoscopic scales. Unfortunately the paper is particularily intricate to read, partly because the problem of equivalence of ensembles is entangled with the analysis of the dynamics, and, as a consequence, the main physical mechanism behind the diffusive scaling does not appear clearly.

With this motivation in mind and with the desire to understand the result by our own methods, we decided to reprove it following the "bisection" technique envisaged in [10].

Our method works as follows. Let $c(L)$ be the largest (over the boundary conditions and number of particles) among the logarithmic Sobolev constants in a cube of side $L$ with given boundary conditions and fixed number of particles. The real hard part is to prove an upper bound for $c(L)$ of the right order; the lower bound is readily obtained by plugging into the logarithmic Sobolev inequality a suitable test function (a slowly varying function of the local density). In order to prove the correct upper bound we look for a recursive inequality of the form

$$
c(2 L) \leqslant \frac{3}{2} c(L)+k L^{2}
$$

which, upon iteration, proves the bound $c(L) \leqslant k^{\prime} L^{2}$.

For this purpose, let $\Lambda$ be the cube of side $2 L$ and let us divide it into two (almost) halves $\Lambda_{1}, \Lambda_{2}$ in such a way that the overlap between $\Lambda_{1}$ and $\Lambda_{2}$ is a thin layer of width $\delta L, \delta \ll 1$. Let us denote by $v$ the canonical Gibbs measure on $\Lambda$ with some given number of particles and let $\operatorname{Ent}_{v}\left(f^{2}\right)=v\left(f^{2} \log \left(f^{2} / v\left(f^{2}\right)\right)\right)$ be the entropy of $f^{2}$ w.r.t. v. If the two $\sigma$-algebras $\mathcal{F}_{1}:=\mathcal{F}_{\Lambda_{1}^{c}}$ and $\mathcal{F}_{2}:=\mathcal{F}_{\Lambda_{2}^{c}}$, namely the $\sigma$-algebras generated by the lattice gas variables outside $\Lambda_{1}$ and $\Lambda_{2}$, respectively, were weakly dependent in the sense that for some $\varepsilon(L) \ll 1$

$$
\left\|v\left(g \mid \mathcal{F}_{2}\right)-v(g)\right\|_{\infty} \leqslant \varepsilon(L) v(g)
$$

for all non-negative functions $g$ measurable w.r.t. $\mathcal{F}_{1}$ (weak dependence on the boundary conditions), then it would follow (see [8] and Section 3.3 below for more details) that (almost factorization of the entropy)

$$
\operatorname{Ent}_{v}\left(f^{2}\right) \leqslant(1+\varepsilon(L)) v\left(\operatorname{Ent}_{v}\left(f^{2} \mid \mathcal{F}_{1}\right)+\operatorname{Ent}_{v}\left(f^{2} \mid \mathcal{F}_{2}\right)\right),
$$

where $\operatorname{Ent}_{v}\left(f^{2} \mid \mathcal{F}_{1}\right)$ is a shorthand notation for the entropy of $f^{2}$ w.r.t. $v\left(\cdot \mid \mathcal{F}_{1}\right)$. Notice that $v\left(\cdot \mid \mathcal{F}_{1}\right)$ is nothing but the canonical measure on the smaller set $\Lambda_{1}$.

If the canonical measure were replaced by the grand canonical one then, under our mixing condition, (1.2) would follow immediately with $\varepsilon(L)=\mathrm{O}\left(\mathrm{e}^{-m \delta L}\right)$ for some positive $m$ and few lines more would suffice to prove uniformly boundedness of the logarithmic Sobolev constant for a "high temperature" Glauber dynamics [8] (see also [16] for a similar discussion for the spectral gap).

For the canonical measure instead, (1.2) cannot hold precisely because of the conservation of the number of particles. Even in the absence of any interaction, the Kawasaki dynamics on two nearby disjoint sets does not factorize into two independent 
dynamics because the particles may migrate from one set to the other one. In particular, the relaxation time in $\Lambda$ (intuitively proportional to $c(L)$ ) is related to the relaxation time of the modified Kawasaki dynamics in which the number of particles in the three sets $\Lambda_{1}, \Lambda_{2}$ and $\Lambda_{1} \cap \Lambda_{2}$ is conserved and to the relaxation time of the process of exchange of particles between $\Lambda_{1}, \Lambda_{2}$ and $\Lambda_{1} \cap \Lambda_{2}$. This suggests to try to separate the two effects which are, a priori, strongly interlaced and to analyze them separately. In some sense this idea is the heart of our approach and technically it can be achieved by elementary conditioning as follows. Let $n_{0}$ and $n_{1}$ be the random variables counting the number of particles in $\Lambda_{1} \cap \Lambda_{2}$ and in $\Lambda \backslash \Lambda_{2}$ respectively and let $\operatorname{Ent}_{v}\left(f^{2} \mid n_{0}, n_{1}\right)$ be the entropy of $f^{2}$ w.r.t. canonical measure $v$ conditioned on $n_{0}, n_{1}$. Then we can write

$$
\operatorname{Ent}_{v}\left(f^{2}\right)=v\left(\operatorname{Ent}_{v}\left(f^{2} \mid n_{0}, n_{1}\right)\right)+\operatorname{Ent}_{v}\left(v\left(f^{2} \mid n_{0}, n_{1}\right)\right) .
$$

The second term in (1.3) can in turn be expanded as

$$
\operatorname{Ent}_{v}\left(v\left(f^{2} \mid n_{0}, n_{1}\right)\right)=v\left(\operatorname{Ent}_{v}\left(v\left(f^{2} \mid n_{0}, n_{1}\right) \mid n_{0}\right)\right)+\operatorname{Ent}_{v}\left(v\left(f^{2} \mid n_{0}\right)\right) .
$$

Notice that in the first term in the r.h.s. of (1.3) we need to bound the entropy with respect a multi canonical measure in which the number of particles in each atom of the partition $\left\{\Lambda \backslash \Lambda_{1}, \Lambda_{1} \cap \Lambda_{2}, \Lambda \backslash \Lambda_{2}\right\}$ is frozen. As shown in [9] (see also Section 3.2 below) such a new measure has better chances to satisfy the "weak dependence" condition (1.2) than the original measure $v$ precisely because of the extra conservation laws. Thus, by the previous reasoning, we may hope to bound the first term in the r.h.s. of (1.3) by the largest among the logarithmic Sobolev constant of each of the three sets times the Dirichlet form of the Kawasaki dynamics. Notice that for each of the three sets the linear dimension in one direction has been (at least) almost halved. Thus the first term in the r.h.s. of (1.3) should be the responsible for the first term in the r.h.s. of (1.1).

Let us now examine the pieces that come from the second term in the r.h.s. of (1.3). As one can observe in (1.4), in each of them one has to bound an entropy with respect to the distribution of a one dimensional discrete random variable e.g. the number of particles $n_{0}$ in the second one. Although such a distribution is difficult to compute exactly, one has a sufficiently good control to be able to establish, via Hardy inequality (see [17] and [2]), a sharp logarithmic Sobolev inequality with respect to the Dirichlet form of a reversible Metropolis birth and death process. Physically such a process corresponds to the creation of an extra particle in e.g. $\Lambda_{1} \cap \Lambda_{2}$ and the contemporary annihilation of a particle in e.g. $\Lambda \backslash \Lambda_{1}$ that is to the exchange of particles among the three sets. Since each particle moves, essentially, by a sort of perturbed random walk, and on average it has to travel a distance $\mathrm{O}(L)$, it is not surprising that the second term in the r.h.s. of (1.3) is the responsible for the $L^{2}$ term in the r.h.s. of (1.2).

Of course and unfortunately, the story is quite involved and we have deliberately hidden here several technical extra conditions that would have obscured the whole discussion without adding any relevant information.

We conclude with some technical comments and a short road map of the paper.

In Sections 5 and 6, that represent the real technical core of the paper, we need to bound from above canonical (and multi canonical) Laplace transform of the form $v_{\Lambda}\left(\exp \left(t \sum_{x \in \Lambda} g_{x}\right)\right)$, where $g_{x}$ is the translated by $x$ of a local function around the origin. 
In principle that could be a difficult task because, due to the conservation law, canonical measures do not have good mixing properties. Instead of entering into the analysis of the large deviations properties of $v_{\Lambda}$, we use the simple, but quite useful bound (see [10]),

$$
v_{\Lambda}\left(\mathrm{e}^{t \sum_{x \in \Lambda} g_{x}}\right) \leqslant A \mu_{\Lambda}\left(\mathrm{e}^{t \sum_{x \in \Lambda} g_{x}}\right),
$$

where $A$ is some universal constant and $\mu_{\Lambda}$ the grand canonical Gibbs measure. Such a bound, valid under our mixing condition, simplifies enormously the problem (compare for instance with [19]) because grand canonical Laplace transforms can be easily bounded using concentration inequalities and logarithmic Sobolev inequalities w.r.t. Glauber dynamics.

Road map. We have decided not to give a completely sequential proof but rather to postpone the proof of some key technical estimates in the second part of the paper. In this way the non-expert reader can read the proof of the main result already in Section 4 provided he accepts some basic bounds that are discussed in Section 3 and proved in Sections 5, 6 and in the appendix. More precisely:

- In Section 2 we define the setting, the mixing condition we need and we state the main result.

- In Section 3 we collect several technical results, part of which are based on the key bounds obtained in Sections 5, 6 and in the appendix, that are necessary to prove the main theorem. A detailed description of these results is given at the beginning of the section.

- In Section 4, by using the results of Section 3, we prove recursively the main theorem on the diffusive scaling of the logarithmic Sobolev constant.

- In Section 5 we study in some detail grand canonical and canonical Laplace transforms via the so called Herbst's approach. This section is the key technical input for the next one.

- In Section 6 we bound the square of covariances of the form $v_{\Lambda}\left(f^{2}, \sum_{x \in \Lambda} g_{x}\right)$, mainly via the entropy inequality and the bounds obtained in Section 5.

- In the appendix we discuss the logarithmic Sobolev inequality for one dimensional random walks via Hardy's inequalities with some application to our problem.

\section{Notation and results}

In this section we first define the setting in which we will work (spin model, Gibbs measure, dynamics), then we define the basic mixing condition on the Gibbs measure and subsequently state the main theorem on this work.

\subsection{The lattice and the configuration space}

The lattice. We consider the $d$ dimensional lattice $\mathbb{Z}^{d}$ with sites $x=\left(x_{1}, \ldots, x_{d}\right)$ and norms

$$
|x|_{p}=\left(\sum_{i=1}^{d}\left|x_{i}\right|^{p}\right)^{1 / p} \quad p \geqslant 1 \text { and }|x|=|x|_{\infty}=\max _{i \in\{1, \ldots, d\}}\left|x_{i}\right| .
$$


The associated distance functions are denoted by $d_{p}(\cdot, \cdot)$ and $d(\cdot, \cdot)$. By $Q_{L}$ we denote the cube of all $x=\left(x_{1}, \ldots, x_{d}\right) \in \mathbb{Z}^{d}$ such that $x_{i} \in\{0, \ldots, L-1\}$. If $x \in \mathbb{Z}^{d}, Q_{L}(x)$ stands for $Q_{L}+x$. We also let $B_{L}$ be the ball (w.r.t. $\left.d(\cdot, \cdot)\right)$ of radius $L$ centered at the origin, i.e. $B_{L}=Q_{2 L+1}((-L, \ldots,-L))$. If $\Lambda$ is a finite subset of $\mathbb{Z}^{d}$ we write $\Lambda \subset \subset \mathbb{Z}^{d}$. The cardinality of $\Lambda$ is denoted by $|\Lambda|$. $\mathbb{F}$ is the set of all nonempty finite subsets of $\mathbb{Z}^{d}$. $[x, y]$ is the closed segment with endpoints $x$ and $y$. The edges of $\mathbb{Z}^{d}$ are those $e=[x, y]$ with $x, y$ nearest neighbors in $\mathbb{Z}^{d}$. We denote by $\mathcal{E}_{\Lambda}$ the set of all edges such that both endpoints are in $\Lambda$.

Given $\Lambda \subset \mathbb{Z}^{d}$ we define its interior and exterior boundaries as respectively, $\partial^{-} \Lambda=$ $\left\{x \in \Lambda: d\left(x, \Lambda^{c}\right) \leqslant 1\right\}$ and $\partial^{+} \Lambda=\left\{x \in \Lambda^{c}: d(x, \Lambda) \leqslant 1\right\}$, and more generally we define the boundaries of width $n$ as $\partial_{n}^{-} \Lambda=\left\{x \in \Lambda: d\left(x, \Lambda^{c}\right) \leqslant n\right\}, \partial_{n}^{+} \Lambda=\left\{x \in \Lambda^{c}: d(x, \Lambda) \leqslant\right.$ $n\}$.

Regular sets. A finite subset $\Lambda$ of $\mathbb{Z}^{d}$ is said to be l-regular, $l \in \mathbb{Z}_{+}$, if $\Lambda$ is the union of a finite number of cubes $Q_{l}\left(x^{i}\right)$ where $x^{i} \in l \mathbb{Z}^{d}$. We denote the class of all such sets by $\mathbb{F}_{l}$. Notice that any set is 1 -regular, i.e. $\mathbb{F}_{l=1}=\mathbb{F}$.

The configuration space. Our configuration space is $\Omega=S^{\mathbb{Z}^{d}}$, where $S=\{0,1\}$, or $\Omega_{V}=S^{V}$ for some $V \subset \mathbb{Z}^{d}$. The single spin space $S$ is endowed with the discrete topology and $\Omega$ with the corresponding product topology. Given $\sigma \in \Omega$ and $\Lambda \subset \mathbb{Z}^{d}$ we denote by $\sigma_{\Lambda}$ the natural projection over $\Omega_{\Lambda}$. If $U, V$ are disjoint, $\sigma_{U} \tau_{V}$ is the configuration on $U \cup V$ which is equal to $\sigma$ on $U$ and $\tau$ on $V$. Given $V \in \mathbb{F}$ we define the number of particles $N_{V}: \Omega \rightarrow \mathbb{N}$ as

$$
N_{V}(\sigma)=\sum_{x \in V} \sigma(x)
$$

while the density is given by $\rho_{V}=N_{V} /|V|$.

If $f$ is a function on $\Omega, \Delta_{f}$ denotes the smallest subset of $\mathbb{Z}^{d}$ such that $f(\sigma)$ depends only on $\sigma_{\Delta_{f}} . f$ is called local if $\Delta_{f}$ is finite. The $l$-support of a function $\Delta_{f}^{(l)}, l \in \mathbb{Z}_{+}$, is the smallest $l$-regular set $V$ such that $\Delta_{f} \subset V . \mathcal{F}_{\Lambda}$ stands for the $\sigma$-algebra generated by the set of projections $\left\{\pi_{x}\right\}, x \in \Lambda$, from $\Omega$ to $\{0,1\}$, where $\pi_{x}: \sigma \mapsto \sigma(x)$. When $\Lambda=\mathbb{Z}^{d}$ we set $\mathcal{F}=\mathcal{F}_{\mathbb{Z}^{d}}$ and $\mathcal{F}$ coincides with the Borel $\sigma$-algebra on $\Omega$ with respect to the topology introduced above. By $\|f\|_{\infty}$ we mean the supremum norm of $f$. The gradient of a function $f$ is defined as

$$
\left(\nabla_{x} f\right)(\sigma)=f\left(\sigma^{x}\right)-f(\sigma),
$$

where $\sigma^{x} \in \Omega$ is the configuration obtained from $\sigma$, by flipping the spin at the site $x$. Finally $\operatorname{Osc}(f)=\sup _{\sigma, \eta}|f(\sigma)-f(\eta)|$.

\subsection{The interaction and the Gibbs measures}

DeFINITION 2.1. - A finite range, translation-invariant potential $\left\{\Phi_{\Lambda}\right\}_{\Lambda \in \mathbb{F}}$ is a collection of real, local functions on $\Omega$ with the following properties

(1) $\Phi_{\Lambda}(\sigma)=\Phi_{\Lambda+x}\left(\theta_{x} \sigma\right)$ for all $\sigma$, all $\Lambda \in \mathbb{F}$ and all $x \in \mathbb{Z}^{d}$, where $\theta_{x}$ denotes the shift operator by $x \in \mathbb{Z}^{d}$. 
(2) For each $\Lambda$ the support of $\Phi_{\Lambda}$ coincides with $\Lambda$.

(3) There exists $r>0$ such that $\Phi_{\Lambda}=0$ if diam $\Lambda>r . r$ is called the range of the interaction.

(4) $\|\Phi\|:=\sum_{\Lambda \ni 0}\left\|\Phi_{\Lambda}\right\|_{\infty}<\infty$.

Given a collection of real numbers $\underline{\lambda}=\left\{\lambda_{x}\right\}_{x \in \mathbb{Z}^{d}}$ and a potential $\Phi$, we define $\Phi \underline{\lambda}$ as

$$
\Phi_{\Lambda}^{\frac{\lambda}{\Lambda}}(\sigma)= \begin{cases}\left(h+\lambda_{x}\right) \sigma(x) & \text { if } \Lambda=\{x\} \\ \Phi_{\Lambda}(\sigma) & \text { otherwise }\end{cases}
$$

where $h$ is the chemical potential (one body part of $\Phi$ ).

Given a potential $\Phi\left(\Phi^{\underline{\lambda}}\right)$ and $V \in \mathbb{F}$, we define the Hamiltonian $H_{V}^{\Phi}: \Omega \mapsto \mathbb{R}$ by

$$
H_{V}^{\Phi}(\sigma)=-\sum_{\Lambda: \Lambda \cap V \neq \emptyset} \Phi_{\Lambda}(\sigma)
$$

For $\sigma, \tau \in \Omega$ we also let $H_{V}^{\Phi, \tau}(\sigma)=H_{V}^{\Phi}\left(\sigma_{V} \tau_{V^{c}}\right)$ and $\tau$ is called the boundary condition. For each $V \in \mathbb{F}, \tau \in \Omega$ the (finite volume) conditional Gibbs measure on $(\Omega, \mathcal{F}$ ), are given by

$$
\mathrm{d} \mu_{V}^{\Phi, \tau}(\sigma)= \begin{cases}\left(Z_{V}^{\Phi, \tau}\right)^{-1} \exp \left[-H_{V}^{\Phi, \tau}(\sigma)\right] & \text { if } \sigma(x)=\tau(x) \text { for all } x \in V^{c} \\ 0 & \text { otherwise }\end{cases}
$$

where $Z_{V}^{\Phi, \tau}$ is the proper normalization factor called partition function. Notice that in (2.2) we have absorbed in the interaction $\Phi$ the usual inverse temperature factor $\beta$ in front of the Hamiltonian. In most notation we will drop the superscript $\Phi$ if that does not generate confusion. Moreover, whenever we consider $\Phi \underline{\lambda}$ instead of $\Phi$, we will write $H_{V}^{\tau, \underline{\lambda}}$ for the finite volume Hamiltonian and $\mu_{V}^{\tau, \underline{\lambda}}$ for the corresponding finite volume Gibbs measure.

Given a measurable bounded function $f$ on $\Omega, \mu_{V}(f)$ denotes the function $\sigma \mapsto$ $\mu_{V}^{\sigma}(f)$ where $\mu_{V}^{\sigma}(f)$ is just the average of $f$ w.r.t. $\mu_{V}^{\sigma}$. Analogously, for any event $X, \mu_{V}^{\tau}(X):=\mu_{V}^{\tau}\left(\mathbb{1}_{X}\right)$, where $\mathbb{1}_{X}$ is the characteristic function of $X . \mu_{V}^{\tau}(f, g)$ stands for the covariance or truncated correlation (with respect to $\mu_{V}^{\tau}$ ) of $f$ and $g$. The set of measures (2.2) satisfies the DLR compatibility conditions

$$
\mu_{\Lambda}^{\tau}\left(\mu_{V}(X)\right)=\mu_{\Lambda}^{\tau}(X) \quad \forall X \in \mathcal{F} \forall V \subset \Lambda \subset \subset \mathbb{Z}^{d}
$$

DEFINITION 2.2. - A probability measure $\mu$ on $(\Omega, \mathcal{F})$ is called a Gibbs measure for $\Phi$ if

$$
\mu\left(\mu_{V}(X)\right)=\mu(X) \quad \forall X \in \mathcal{F} \forall V \in \mathbb{F}
$$

see e.g. [13].

We introduce the canonical Gibbs measures on $(\Omega, \mathcal{F})$ defined as

$$
v_{\Lambda, N}^{\tau}=\mu_{\Lambda}^{\tau}\left(\cdot \mid N_{\Lambda}=N\right) \quad N \in\{0,1, \ldots,|\Lambda|\}
$$




\subsection{The dynamics}

We consider the so-called Kawasaki dynamics in which particles (spins with $\sigma(x)=$ $+1)$ can jump to nearest neighbor empty $(\sigma(x)=0)$ locations, keeping the total number of particles constant. For $\sigma \in \Omega$, let $\sigma^{x y}$ be the configuration obtained from $\sigma$ by exchanging the spins $\sigma(x)$ and $\sigma(y)$. Let $t_{x y} \sigma=\sigma^{x y}$ and define $\left(T_{x y} f\right)(\sigma)=f\left(t_{x y} \sigma\right)$. The stochastic dynamics we want to study is determined by the Markov generators $L_{V}, V \subset \subset \mathbb{Z}^{d}$, defined by

$$
\left(L_{V} f\right)(\sigma)=\sum_{[x, y] \in \mathcal{E}_{V}} c_{x y}(\sigma)\left(\nabla_{x y} f\right)(\sigma) \quad \sigma \in \Omega, f: \Omega \mapsto \mathbb{R},
$$

where $\nabla_{x y}=T_{x y}-\mathbb{1}$. The nonnegative real quantities $c_{x y}(\sigma)$ are the transition rates for the process.

The general assumptions on the transition rates are

(1) Finite range. $c_{x y}(\sigma)$ depends only on the spins $\sigma(z)$ with $d(\{x, y\}, z)<r$.

(2) Detailed balance. For all $\sigma \in \Omega$ and $[x, y] \in \mathcal{E}_{\mathbb{Z}^{d}}$

$$
\exp \left[-H_{\{x, y\}}(\sigma)\right] c_{x y}(\sigma)=\exp \left[-H_{\{x, y\}}\left(\sigma^{x y}\right)\right] c_{x y}\left(\sigma^{x y}\right)
$$

(3) Positivity and boundedness. There exist positive real numbers $c_{m}(\beta)$ and $c_{M}(\beta)$ such that

$$
c_{m} \leqslant c_{x y}(\sigma) \leqslant c_{M} \quad \forall x, y \in \mathbb{Z}^{d}, \sigma \in \Omega .
$$

We denote by $L_{V, N}^{\tau}$ the operator $L_{V}$ acting on $L^{2}\left(\Omega, v_{V, N}^{\tau}\right)$ (this amounts to choosing $\tau$ as the boundary condition and $N$ as the number of particles). Assumptions (1), (2) and (3) guarantee that there exists a unique Markov process whose generator is $L_{V, N}^{\tau}$, and whose semigroup we denote by $\left(T_{t}^{V, N, \tau}\right)_{t \geqslant 0} . L_{V, N}^{\tau}$ is a bounded operator on $L^{2}\left(\Omega, v_{V, N}^{\tau}\right)$ and $v_{V, N}^{\tau}$ is its unique invariant measure. Moreover $v_{V, N}^{\tau}$ is reversible with respect to the process, i.e. $L_{V, N}^{\tau}$ is self-adjoint on $L^{2}\left(\Omega, v_{V, N}^{\tau}\right)$.

A first fundamental quantity associated with the dynamics of a reversible system is the spectral gap of the generator, i.e.

$$
\operatorname{gap}\left(L_{V, N}^{\tau}\right)=\inf \operatorname{spec}\left(-L_{V, N}^{\tau}\left\lceil\mathbb{1}^{\perp}\right)\right.
$$

where $\mathbb{1}^{\perp}$ is the subspace of $L^{2}\left(\Omega, v_{V, N}^{\tau}\right)$ orthogonal to the constant functions. We let $\mathcal{E}$ to be the Dirichlet form associated with the generator $L_{V, N}^{\tau}$,

$$
\mathcal{E}_{V, N}^{\tau}(f, f)=\left\langle f,-L_{V, N}^{\tau} f\right\rangle_{L^{2}\left(\Omega, v_{V, N}^{\tau}\right)}=\frac{1}{2} \sum_{[x, y] \in \mathcal{E}_{V}} v_{V, N}^{\tau}\left[c_{x y}\left(\nabla_{x y} f\right)^{2}\right]
$$

and $\operatorname{Var}_{V, N}^{\tau}$ the variance relative to the probability measure $v_{V, N}^{\tau}$. Then the gap can also be characterized as

$$
\operatorname{gap}\left(L_{V, N}^{\tau}\right)=\inf _{\substack{f \in L^{2}\left(\Omega, v_{V, N}^{\tau}\right) \\ \operatorname{Var}_{V, N}^{\tau}(f) \neq 0}} \frac{\mathcal{E}_{V, N}^{\tau}(f, f)}{\operatorname{Var}_{V, N}^{\tau}(f)}
$$


A second relevant quantity is the logarithmic Sobolev constant $c_{V, N}^{\tau}$ defined as the smallest constant $c$ such that

$$
\operatorname{Ent}_{V, N}^{\tau}\left(f^{2}\right) \leqslant \frac{c}{2} \mathcal{E}_{V, N}^{\tau}(f, f)
$$

for all non-negative functions $f$ with $v_{V, N}^{\tau}\left(f^{2}\right)=1$, where $\operatorname{Ent}_{V, N}^{\tau}\left(f^{2}\right)=v_{V, N}^{\tau}\left(f^{2} \ln f^{2}\right)$. For the connection between spectral gap, logarithmic Sobolev constant and speed of relaxation to equilibrium we refer the reader to [12].

\subsection{Definition of the mixing condition and main results}

In order to formulate our basic mixing condition on the two (or more) body part of the interaction $\Phi$ we fix positive numbers $C, m, l$ with $l \in \mathbf{N}$. We then say that a collection of real numbers $\underline{\lambda}:=\left\{\lambda_{x}\right\}_{x \in \mathbb{Z}^{d}}$ is $l$-regular if, for all $i \in \mathbb{Z}^{d}$, and all $x \in Q_{l}\left(x^{i}\right), x^{i} \in l \mathbb{Z}^{d}$, $\lambda_{x}=\lambda_{x^{i}}$.

DEFINITION OF PROPERTY USMT $(C, m, l)$. - For any l-regular set $\Lambda$, any l-regular $\underline{\lambda}$, any boundary condition $\tau$ and any pair of bounded local functions $f$ and $g$

$$
\left|\mu_{\Lambda}^{\tau, \underline{\lambda}}(f, g)\right| \leqslant C \sup _{\tau} \mu_{\Lambda}^{\tau, \underline{\lambda}}(|f|) \sup _{\tau} \mu_{\Lambda \backslash \Delta_{f}^{(l)}}^{\tau, \underline{\lambda}}(|g|) \sum_{x \in \partial_{r}^{-} \Delta_{f}^{(l)}} \sum_{y \in \partial_{r}^{-} \Delta_{g}^{(l)}} \mathrm{e}^{-m|x-y|}
$$

provided that $d\left(\Delta_{f}^{(l)}, \Delta_{g}^{(l)}\right) \geqslant l$. Here $r$ denotes the range of the interaction.

Remark. - The expert reader may have noticed that our condition is different, and in principle stronger, than the one used in [14] and [19] because we require the exponential decay of covariances uniformly in the chemical potential even when the latter varies over the atoms of a partition of $\Lambda$ while in the above mentioned papers the chemical potential is assumed to be constant over $\Lambda$. In two dimension, one can prove by cluster expansion methods (see [4]), that the two conditions are equivalent. In higher dimension one can construct examples (see Appendix A2 of [4]) in which a kind of phase transition occurs along the interface between two subsets with different chemical potential, even if for all $l$-regular sets $\Lambda$ the covariances decay exponentially fast uniformly w.r.t. to constant chemical potentials.

We are finally in a position to formulate the main result of this paper on the logarithmic Sobolev constant of Kawasaki dynamics in a finite volume.

THEOREM 2.3. - Assume that there exist positive numbers $C, m, l$, with $l \in \mathbf{N}$, such that property $U S M T(C, m, l)$ holds. Then there exist positive constants $c_{1}, c_{2}$ such that

$$
c_{1} L^{2} \leqslant c_{Q_{L}, N}^{\tau} \leqslant c_{2} L^{2}
$$

for all boundary condition $\tau$ and particle number $N$. 


\section{Technical results}

Here we collect several preliminary technical results that will be used in the future. Although the reader may skip this section during a first reading and come back when these results are needed, we still think it is useful to give a short roadmap of the section.

- In Sections 3.1, 3.2 we recall some useful results on the comparison between finite volume multicanonical Gibbs measures, namely grand canonical Gibbs measures conditioned to have a specified number of particles in the atoms of a given partition of a finite set $\Lambda$, and the corresponding unconditioned measures.

- In Section 3.3, we prove a "baby" version of a nice, new inequality for the entropy (see [8]), that, very roughly speaking, allows us to bound the multicanonical entropy in terms of the multicanonical average of the sum of local entropies and of the multicanonical variance. For the expert reader we just mention that the importance of such an inequality resides in the fact that one is spared from the cumbersome computation of quantities like $\left[\nabla_{x y} v_{\Lambda}^{\tau}\left(f^{2}\right)^{1 / 2}\right]^{2}$.

- In Sections 3.4, 3.5, 3.6, we first show how to compute and then how to estimate quantities like $v\left(\left[\frac{\mathrm{d}}{\mathrm{d} n} v\left(f^{2} \mid N_{V}=n\right)^{1 / 2}\right]^{2}\right)$, where $v$ is a multi canonical measure over the atoms $\left\{\Lambda_{i}\right\}_{i=1}^{k}$ of a partition of a finite set $\Lambda, V$ is a subset of a given atom $\Lambda_{j}$ and $\frac{\mathrm{d}}{\mathrm{d} n}$ is the discrete derivative. As it will appear clearly in Section 6 , terms like the one above naturally appear in the recursive bound of the spectral gap and, roughly speaking, they measure the influence on the logarithmic Sobolev constant of the exchange of particles between different atoms of the partition.

- In Section 3.7 we recall some results proved in [10] on the distribution of the number of particles in an atom of a given partition of a finite set $\Lambda$ under a multicanonical measure. These results will then be used to prove sharp bound on the logarithmic Sobolev inequality for such a measure via Hardy inequality (see the appendix).

\section{The general setting}

Throughout all this section our setting and notation will be as follows.

Fix $\delta_{0} \in(0,1)$ and two integers $j_{\max }, l$ such that $\delta_{0} j_{\max }<1$. Let $L_{1}, \ldots, L_{j \max }$ be large multiples of the basic length scale $l$, let $L=\sum_{j} L_{j}$ and assume that $L_{j} \geqslant \delta_{0} L$ for any $j$. We then choose one coordinate direction, e.g. the $d$ direction, and we take $\Lambda=Q_{L}, \Lambda_{1}$ equal to the first slice of $\Lambda$ orthogonal to $d$-direction of width $L_{1}$, i.e. $\Lambda_{1}=\left\{x \in \Lambda: 0 \leqslant x_{d}<L_{1}\right\}, \Lambda_{2}$ equal to the slice of $\Lambda$ on top of $\Lambda_{1}$ of width $L_{2}$ and so on. Let also $\mathbf{N}=\left\{N_{j}\right\}_{j=1}^{j_{\max }}$ be a set of possible values of $\mathbf{N}_{\Lambda}:=\left\{N_{\Lambda_{j}}\right\}_{j=1}^{j_{\max }}, \rho_{j}=\frac{N_{j}}{\left|\Lambda_{j}\right|}$ the corresponding densities and let us assume, for a given boundary condition $\tau$, that $\underline{\lambda}=\underline{\lambda}(\tau, \mathbf{N})$ is constant on each set $\Lambda_{j}$ and such that $\mu_{\Lambda}^{\tau, \underline{\lambda}}\left(N_{\Lambda_{j}}\right)=N_{j}, j=1, \ldots, j_{\max }$ (see the appendix of [9] for a proof of the existence of $\underline{\lambda}$ ). Notice that, as far as the tilting fields $\underline{\lambda}$ is concerned, the variables $N_{j}$ could also be any set of real numbers in the range $\left[0,\left|\Lambda_{j}\right|\right]$. In particular we will be allowed to compute the derivative of $\underline{\lambda}$ w.r.t. any of them. We simply write $\mu$ for $\mu_{\Lambda}^{\tau, \underline{\lambda}}$ and $v$ for the multi canonical Gibbs measure $\mu\left(\cdot \mid N_{\Lambda_{j}}=N_{j}, j=1, \ldots, j_{\max }\right)$. 
Finally, in all this section $k$ will denote a generic constant, depending on the interaction, on the dimensions of the lattice, on $\delta_{0}$ and on $j_{\max }$, whose value may vary from line to line.

\subsection{On the tilting fields}

We begin by recalling the following quite general result on the relationship between particle numbers, boundary condition and the chemical potential (see the appendix in [9]). We assume here $j_{\max }=1$ so that we can set, for notation convenience, $N_{1}=$ $n, \rho=\frac{n}{L^{d}}$. In order to be more clear, in the following lemma we will write explicitly the dependence on the boundary conditions and the chemical potential of the grand canonical Gibbs state.

LEMmA 3.1. - Assume property $\operatorname{USMT}(C, m, l)$ and let $\alpha \in(0,1)$. Then there exists a constant $k$ independent on $L$ such that for any $L$ large enough and any $f$ with $\|f\|_{\infty}=1$.

(1) If $d\left(\Delta_{f}, \Lambda^{c}\right) \geqslant L^{\alpha}$

$$
\begin{aligned}
& \text { (i) } \sup _{y \in \partial_{r}^{+} \Lambda}\left\|\nabla_{y} \mu^{\tau, \lambda}(f)\right\|_{\infty} \leqslant k \rho \frac{\left|\Delta_{f}\right|}{|\Lambda|}, \\
& \text { (ii) } \sup _{y \in \partial_{r}^{+} \Lambda}\left\|\nabla_{y} \frac{\mathrm{d}}{\mathrm{d} n} \mu^{\tau, \lambda}(f)\right\|_{\infty} \leqslant k \frac{\left|\Delta_{f}\right|}{|\Lambda|^{2}} .
\end{aligned}
$$

(2) If $\Delta_{f} \subset \Lambda$

$$
\begin{aligned}
& \text { (i) }\left\|\frac{\mathrm{d}}{\mathrm{d} n} \mu^{\tau, \lambda}(f)\right\|_{\infty} \leqslant k \frac{\left|\Delta_{f}\right|}{|\Lambda|}, \\
& \text { (ii) }\left\|\frac{\mathrm{d}^{2}}{\mathrm{~d} n^{2}} \mu^{\tau, \lambda}(f)\right\|_{\infty} \leqslant k \frac{1}{n} \frac{\left|\Delta_{f}\right|}{|\Lambda|} .
\end{aligned}
$$

Proof. - We omit the proof since is practical the same of an almost identical result (Proposition 3.1) of [10].

\subsection{Equivalence of ensembles}

Here we recall some fine results on the finite volume equivalence of ensembles that will be crucial in most of our future arguments. We refer the reader to Sections 5, 7.2 and 7.3 of [9].

Given $M>0, \varepsilon \in(0,1)$ and $\Delta \subset \Lambda$, we say that $\Delta$ is good if there exists $j \in\left[1, j_{\max }\right]$ such that either

$$
\begin{gathered}
\{x \in \Lambda: d(x, \Delta) \leqslant M \ln L\} \subset \Lambda_{j} \text { and } \rho_{j} \geqslant|\Lambda|^{-\varepsilon} \\
\text { or } \\
\{x \in \Lambda: d(x, \Delta) \leqslant M\} \subset \Lambda_{j} \text { and } \rho_{j} \leqslant|\Lambda|^{-\varepsilon} .
\end{gathered}
$$


A set is bad if it is not good. For good sets $\Delta \subset \Lambda_{j}, j=1, \ldots, j_{\max }$, we define

$$
\bar{\Delta}= \begin{cases}\{x \in \Lambda: d(x, \Delta) \leqslant M \ln L\} & \text { if } \rho_{j} \geqslant|\Lambda|^{-\varepsilon}, \\ \{x \in \Lambda: d(x, \Delta) \leqslant M\} & \text { if } \rho_{j} \leqslant|\Lambda|^{-\varepsilon},\end{cases}
$$

which for bad sets

$$
\bar{\Delta}=\{x \in \Lambda: d(x, \Delta) \leqslant M \ln L\} .
$$

With these notations the results on the finite volume equivalence of ensembles that will be essential for the rest of this paper read as follows.

Proposition 3.2. - Assume condition $\operatorname{USMT}(C, m, l)$. Then, for any $l, M$ large enough and $\varepsilon$ small enough independent of $\left\{\rho_{j}\right\}_{j=1}^{j_{\max }}$, there exists $L_{0}=L_{0}(C, m,\|\Phi\|, l$, $\left.M, \delta_{0}, \varepsilon\right)$ such that, for any $L \geqslant L_{0}$ the following holds. Fix two l-regular sets $\Delta_{1}, \Delta_{2}$ in $\Lambda$ such that $\left|\Delta_{i}\right| \leqslant|\Lambda|^{1-\varepsilon}, i=1,2$, and two functions $f, g$ with $\|f\|_{\infty}=\|g\|_{\infty}=1$, $\Delta_{f}^{(l)}=\Delta_{1}, \Delta_{g}^{(l)}=\Delta_{2}$.

(1) Assume $j_{\max }=1$. Then

$$
|v(f, g)| \leqslant C(f, g) \rho\left[\frac{1}{|\Lambda|}+\mathrm{e}^{-m d\left(\Delta_{1}, \Delta_{2}\right)}\right],
$$

where $C(f, g)=k\left|\Delta_{1}\right|^{2}\left|\Delta_{2}\right|^{2}$. Moreover

$$
|v(f)-\mu(f)| \leqslant k \frac{\left|\Delta_{1}\right|}{|\Lambda|} .
$$

(2) Assume $j_{\max } \geqslant 2$. Then

$$
|v(f, g)| \leqslant C(f, g) A\left(\Delta_{1}, \Delta_{2}\right) \begin{cases}v(|f|) \wedge v(|g|) & \text { if } \Delta_{1} \text { and } \Delta_{2} \text { are both good } \\ & \text { or bad, } \\ v(|f|) & \text { if } \Delta_{1} \text { is bad, } \\ v(|g|) & \text { if } \Delta_{2} \text { is bad, }\end{cases}
$$

where

$$
A\left(\Delta_{1}, \Delta_{2}\right)= \begin{cases}\frac{1}{|\Lambda|}+\mathrm{e}^{-m d\left(\Delta_{1}, \Delta_{2}\right)} & \text { if } \Delta_{1} \text { or } \Delta_{2} \text { is good, } \\ \frac{1}{|\Lambda|}\left(\frac{\left|\bar{\Delta}_{2}\right|}{\left|\Delta_{2}\right|}\right)^{2}\left(\frac{\left|\bar{\Delta}_{1}\right|}{\left|\Delta_{1}\right|}\right)^{2}+\mathrm{e}^{-m d\left(\Delta_{1}, \Delta_{2}\right)} & \text { otherwise. }\end{cases}
$$

(3) Assume $j_{\max } \geqslant 2$ and $\Delta_{1} \subset \Lambda_{j}, j \leqslant j_{\max }$. Then

$$
\sup _{\tau^{\prime} \in \Omega_{\tau}}\left|v^{\tau}(f)-v^{\tau^{\prime}}(f)\right| \leqslant k \begin{cases}\left|\Delta_{1}\right|\left[\frac{1}{|\Lambda|}+\frac{1}{L^{k-n+1}}\right] & \text { if } \Delta_{1} \text { is good, } \\ \frac{\left|\Delta_{1}\right|}{|\Lambda|}\left(\frac{\left|\bar{\Delta}_{1}\right|}{\left|\Delta_{1}\right|}\right)^{2}+\max _{j=n, n \pm 1}\left[\frac{\left|\bar{\Delta}_{1} \cap \Lambda_{j}\right|^{\frac{1}{2}}}{L^{j \max }-j+1}\right] & \text { if } \Delta_{1} \text { is bad, }\end{cases}
$$

where $\Omega_{\tau}$ is the set of configurations $\tau^{\prime}$ that coincide with $\tau$ in the half space $\left\{x \in \mathbb{Z}^{d}: x_{d}<L\right\}$. 
Remark. - Actually the first part of the proposition holds in a much more general geometric context (see Section 7.3 of [9]).

For future purposes the next result is stated in a slightly more general form. The proof is given in [10].

Proposition 3.3. - Assume condition $\operatorname{USMT}(C, m, l)$ and let $f$ be such that $\left|\Lambda_{j} \backslash \Delta_{f}\right| \geqslant \delta_{0}\left|\Lambda_{j}\right|$ for any $j=1, \ldots, j_{\max }$. Then, for any $l$ large enough, there exists $a$ constant $A$ depending only on $C, m,\|\Phi\|, l, j_{\max }, \delta_{0}$ such that

$$
v(|f|) \leqslant A \mu(|f|) .
$$

\section{In particular}

$$
\operatorname{Var}_{v}(f, f) \leqslant A \operatorname{Var}_{\mu}(f, f) .
$$

We conclude this paragraph with a final result that plays a crucial role in our approach (see Section 3.3). For simplicity we consider only the two dimensional case and at the end we explain how to generalize it to higher dimensions.

Assume that the number of layers $j_{\max }$ is greater than 4 , fix $3 \leqslant j_{0} \leqslant j_{\max }$ and let $A=\bigcup_{j=1}^{j_{0}} \Lambda_{j}, B=\bigcup_{j_{0}-1}^{j_{\max }} \Lambda_{j}$ and $S=\Lambda_{j_{0}-1} \cup \Lambda_{j_{0}}$. Let also $v_{X}(\cdot):=v\left(\cdot \mid \mathcal{F}_{X^{c}}\right)$ for $X=A, B, S$. Notice that $\nu$-almost surely $N_{A}, N_{B}$ and $N_{S}$ are constant.

LEMMA 3.4. - Assume condition $\operatorname{USMT}(C, m, l)$ and let $g$ be a positive function measurable w.r.t. $\mathcal{F}_{A^{c}}$. Then for any $\varepsilon>0$ there exists $L_{0}=L_{0}\left(\varepsilon, C, m,\|\Phi\|_{\infty}, l, \delta\right)$ such that, if $L \geqslant L_{0}$,

$$
\left\|v_{B}(g)-v(g)\right\|_{\infty} \leqslant \varepsilon v(g) .
$$

In other words the Radon-Nykodim derivative of the marginal on $\mathcal{F}_{A^{c}}$ of $v_{B}$ w.r.t. the same marginal of $v$ is close to one in the $L^{\infty}$-norm.

Proof. - Fix $\varepsilon \in(0,1)$ and $\eta$ and let $h_{x}(n):=\mathrm{e}^{-\nabla_{x} H(\eta)} / \nu_{B}^{\eta}\left(\mathrm{e}^{-\nabla_{x} H}\right)$. Using the definition of $h_{x}$ and the DLR equations (valid because the numbers of particles in $A, B, S$ are constant) we have

$$
\begin{aligned}
\left|v_{B}^{\eta^{x}}(g)-v_{B}^{\eta}(g)\right| & =\left|v_{B}^{\eta}\left(g, h_{x}\right)\right| \\
& =\left|v_{B}^{\eta}\left(g, v_{S}\left(h_{x}\right)\right)\right| \\
& \leqslant v_{B}^{\eta}(g) \sup _{\tau^{\prime} \in \Omega_{\tau}}\left|v_{S}^{\tau}\left(h_{x}\right)-v_{S}^{\tau^{\prime}}\left(h_{x}\right)\right|,
\end{aligned}
$$

where $\Omega_{\tau}$ is the set of configurations $\tau^{\prime}$ which differs from $\tau$ only on $\partial^{+} S \cap B$. By point (3) of Proposition 3.2 there exists a positive constant $k$ such that

$$
\sup _{\tau^{\prime} \in \Omega_{\tau}}\left|v_{S}^{\tau}\left(h_{x}\right)-v_{S}^{\tau^{\prime}}\left(h_{x}\right)\right| \leqslant k \frac{(\ln L)^{\frac{1}{2}}}{L^{2}} .
$$

Thus

$$
v_{B}^{\eta^{x}}(g) \leqslant\left[1+k \frac{(\ln L)^{\frac{1}{2}}}{L^{2}}\right] v_{B}^{\eta}(g) .
$$


As any two boundary configurations $\eta$ and $\eta^{\prime}$ differ at most in $k L$ sites, by iterating (3.3), we have

$$
v_{B}^{v}(g) \leqslant\left[1+C^{\prime} \frac{(\ln L)^{\frac{1}{2}}}{L^{2}}\right]^{k L} v_{B}^{\eta^{\prime}} g \leqslant(1+\varepsilon) v_{B}^{\eta^{\prime}}(g)
$$

for $L$ large enough. It suffices now to integrate (3.4) w.r.t. $\mathrm{d} v\left(\eta^{\prime}\right)$ and use the arbitrariness of $\eta$.

Remark. - The restriction of $d=2$ comes from point (3) of Proposition 3.2. In fact, in e.g. three dimensions, the bound (3.2) becomes useless. The way out is to have the "safety belt" $S$ divided into more layers (just three in $d=3$ ). It is interesting at this point to observe that a similar problem occurs also in the recursive study of the spectral gap (see [10]). In that case however, the safety belt $S$ in $d=2$ consisted of just one atom and not of two as in our case. The reason is that, in the spectral gap analysis, a weaker form of Lemma 3.4 was necessary in which the r.h.s. of the basic inequality is $\varepsilon\|g\|_{\infty}$ and not $\varepsilon v(g)$.

\subsection{A two-block inequality on the entropy}

Here we give a result that is a key step in our recursive bound of the spectral gap of Kawasaki dynamics. For simplicity we discuss the next estimates in two dimensions and at the end we explain how to generalize it to higher dimensions.

Assume that the number of layers $j_{\max }$ is greater than 4 , fix $3 \leqslant j_{0} \leqslant j_{\max }$ and let $A=\bigcup_{j=1}^{j_{0}} \Lambda_{j}, B=\bigcup_{j_{0}-1}^{j_{\max }} \Lambda_{j}$ and $S=\Lambda_{j_{0}-1} \cup \Lambda_{j_{0}}$. Let also $v_{X}(\cdot):=v\left(\cdot \mid \mathcal{F}_{X^{c}}\right)$ for $X=A, B, S$. Notice that $v$-almost surely $N_{A}, N_{B}$ and $N_{S}$ are constant.

Proposition 3.5. - Assume USMT $(C, m, l)$. Then, for any $\varepsilon>0$ there exists $L_{0}$ depending only on $\varepsilon, C, m,\|\Phi\|_{\infty}, l, \delta$ such that, if $L \geqslant L_{0}$,

$$
\operatorname{Ent}_{v}\left(f^{2}\right) \leqslant v\left(\operatorname{Ent}_{v_{A}}\left(f^{2}\right)+\operatorname{Ent}_{v_{B}}\left(f^{2}\right)\right)+\varepsilon \operatorname{Var}_{v}(f)
$$

for any $f$ with $v(f)=0$.

Remark. - The origin of this inequality is the following simple bound valid for an arbitrary product measure $v=v_{1} \otimes v_{2}$ :

$$
\operatorname{Ent}_{v}\left(f^{2}\right) \leqslant v\left(\operatorname{Ent}_{v_{1}}\left(f^{2}\right)+\operatorname{Ent}_{v_{2}}\left(f^{2}\right)\right) \quad \forall f \geqslant 0
$$

and, in some sense, the term $\varepsilon \operatorname{Var}_{v}(f)$ in (3.5) takes into account the correction due to the presence of the overlapping strip $S$. As we will see the proof of the proposition is rather simple. Quite a non-trivial task is to remove the restriction $v(f)=0$. If one tries to use the Rothaus inequality (see e.g. [2])

$$
\operatorname{Ent}_{v}\left(f^{2}\right) \leqslant 2 \operatorname{Var}_{v}(f)+\operatorname{Ent}_{v}\left([f-v(f)]^{2}\right)
$$

together with (3.5), one gets immediately

$$
\operatorname{Ent}_{v}\left(f^{2}\right) \leqslant v\left(\operatorname{Ent}_{v_{A}}\left(f^{2}\right)+\operatorname{Ent}_{v_{B}}\left(f^{2}\right)\right)+(2+\varepsilon) \operatorname{Var}_{v}(f)
$$


which is much worse than (3.5) because of the factor $2+\varepsilon$. Quite remarkably, in [8] the restriction $v(f)=0$ has been removed in great generality and moreover the error term $\varepsilon \operatorname{Var}_{v}(f)$ has been replaced by $\varepsilon \operatorname{Ent}_{v}\left(f^{2}\right)$. The resulting inequality has been then applied to provide a very simple and natural proof of the logarithmic Sobolev inequality for grand canonical Gibbs measures under a mixing condition. For a "variance" counterpart of (3.5) (that is $\operatorname{Ent}_{v}\left(f^{2}\right) \mapsto \operatorname{Var}_{v}(f)$ ) we refer the reader to Proposition 3.4 in [10] (see also Section 3.7 in [16] and [3] for the Glauber case).

Proof. - The proof is based on Lemma 3.4 above. We write

$$
\begin{aligned}
\operatorname{Ent}_{v}\left(f^{2}\right)= & v\left(f^{2} \log \frac{f^{2}}{v_{A}\left(f^{2}\right)}\right)+v\left(f^{2} \log \frac{v_{A}\left(f^{2}\right)}{v_{B}\left(v_{A}\left(f^{2}\right)\right)}\right) \\
& +v\left(f^{2} \log \frac{v_{B}\left(v_{A}\left(f^{2}\right)\right)}{v\left(f^{2}\right)}\right) .
\end{aligned}
$$

The first term in (3.7) is equal to $v\left(\operatorname{Ent}_{v_{A}}\left(f^{2}\right)\right)$. By using the variational definition of the entropy $\operatorname{Ent}_{v_{B}}\left(f^{2}\right)$

$$
\operatorname{Ent}_{v_{B}}\left(f^{2}\right)=\sup _{g: v_{B}\left(e^{g}\right)=1} v_{B}\left(f^{2} g\right)
$$

together with

$$
v_{B}\left(\frac{v_{A}\left(f^{2}\right)}{v_{B}\left(v_{A}\left(f^{2}\right)\right)}\right)=1
$$

the second term in (3.7) can be bounded from above by $v\left(\operatorname{Ent}_{v_{B}}\left(f^{2}\right)\right)$. Finally, the argument of the logarithm in the third term is smaller than

$$
v_{B}\left(\frac{v_{A}\left(f^{2}\right)}{v\left(f^{2}\right)}\right)=\frac{v_{B}\left(v_{A}\left(f^{2}\right)\right)}{v\left(v_{A}\left(f^{2}\right)\right)} \leqslant 1+\varepsilon
$$

for any $L$ large enough independent of $f$ because of Lemma 3.4. Thus the third term can be bounded from above by $\varepsilon v\left(f^{2}\right)=\varepsilon \operatorname{Var}(f)$.

Remark. - It is clear from the proof that the key input for the result is Lemma 3.4. Therefore one can easily formulate the proposition in dimension greater than two simply by assuming that the set $S$ consists of a sufficiently large number of layers (just three in $d=3$ ) as it was explained already in the remark after the proof of Lemma 3.4.

\subsection{Computing the gradient with respect to the particle number}

Let $V$ and $W$ be such that $V \cap W=\emptyset, \Lambda_{j}=V \cup W$ for some $j \in\left\{1, \ldots, j_{\max }\right\}$ and $0<\delta_{0} \leqslant \frac{|V|}{|W|} \leqslant \delta_{0}^{-1}$. Here $\delta_{0}$ is the same as the one entering in the general multicanonical setting described at the beginning of the section.

Our goal is to compute, for an arbitrary $f$, the gradient w.r.t. to $n$ of $\left(v\left(f^{2} \mid N_{V}=\right.\right.$ $n))^{1 / 2}$. For simplicity we set $v(\cdot \mid n):=v\left(\cdot \mid N_{V}=n\right)$ and $\gamma(n):=v\left(N_{V}=n\right)$.

For $x, z \in \mathbb{Z}^{d}$, we define the events

$$
E_{x z}=\{\sigma \in \Omega: \sigma(x)=1, \sigma(z)=0\} .
$$


In Proposition 3.10 of [10] the following result was proved.

Proposition 3.6. - Let $V$ and $W$ be such that $V \cap W=\emptyset$ and $\Lambda_{j}=V \cup W$ for some $j \in\left\{1, \ldots, j_{\max }\right\}$. Let also $c_{n}=n\left(|W|-N_{j}+n\right)$, that is (number of particles in $V$ ) $\times$ (number of holes in $W$ ), and let $c_{n}^{\prime}=n\left(|V|-N_{j}+n\right)$. Then, for all functions $f$ on $\Omega$ we have

(1)

$$
\begin{aligned}
v\left(f^{2} \mid n\right)-v\left(f^{2} \mid n-1\right)= & \left.\frac{1}{c_{n}} \frac{\gamma(n-1)}{\gamma(n)} \sum_{\substack{x \in V \\
z \in W}} v\left(\left(\nabla_{z x} f^{2}\right)\right) \mathbb{1}_{E_{z x}} \mathrm{e}^{-\nabla_{x z} H_{\Lambda}} \mid n-1\right) \\
& +\frac{1}{c_{n}} \frac{\gamma(n-1)}{\gamma(n)} \sum_{\substack{x \in V \\
z \in W}} v\left(\left(\mathrm{e}^{-\nabla_{x z} H_{\Lambda}}-1\right) \mathbb{1}_{E_{z x}}, f^{2} \mid n-1\right),
\end{aligned}
$$

(2)

$$
\begin{aligned}
v\left(f^{2} \mid n\right)-v\left(f^{2} \mid n-1\right)= & -\frac{1}{c_{N-n+1}^{\prime}} \frac{\gamma(n)}{\gamma(n-1)} \sum_{\substack{x \in V \\
z \in W}} v\left(\left(\nabla_{x z} f^{2}\right) \mathbb{1}_{E_{x z}} \mathrm{e}^{-\nabla_{x z} H_{\Lambda}} \mid n\right) \\
& +\frac{1}{c_{N-n+1}^{\prime}} \frac{\gamma(n)}{\gamma(n-1)} \sum_{\substack{x \in V \\
z \in W}} v\left(\left(\mathrm{e}^{-\nabla_{x z} H_{\Lambda}}-1\right) \mathbb{1}_{E_{x z}}, f^{2} \mid n\right) .
\end{aligned}
$$

Let now $n_{\max }, n_{\min }$ be the maximum and minimum value of the particle number in $V$ under the constraint that $N_{\Lambda_{j}}=N_{j}$. Let $u=\left\lfloor\rho_{j}|V|\right\rfloor$, where $\rho_{j}=\frac{N_{j}}{\left|\Lambda_{j}\right|}$, and let, for $n \in\left(n_{\min }, n_{\max }\right)$,

$$
\begin{gathered}
A(n)= \begin{cases}\frac{1}{c_{n}} \frac{\gamma(n-1)}{\gamma(n)} \sum_{\substack{x \in V \\
z \in W}} v\left(\left(\nabla_{z x} f^{2}\right) \mathbb{1}_{E_{z x}} \mathrm{e}^{-\nabla_{x z} H_{\Lambda}} \mid n-1\right) & \text { if } n \leqslant u, \\
\frac{1}{c_{N-n+1}^{\prime}} \frac{\gamma(n)}{\gamma(n-1)} \sum_{\substack{x \in V \\
z \in W}} v\left(\left(\nabla_{x z} f^{2}\right) \mathbb{1}_{E_{x z}} \mathrm{e}^{-\nabla_{x z} H_{\Lambda}} \mid n\right) & \text { otherwise, }\end{cases} \\
B(n)= \begin{cases}\frac{1}{c_{n}} \frac{\gamma(n-1)}{\gamma(n)} \sum_{\substack{x \in V \\
z \in W}} v\left(\left(\mathrm{e}^{-\nabla_{x z} H_{\Lambda}}-1\right) \mathbb{1}_{E_{z x}}, f^{2} \mid n-1\right) & \text { if } n \leqslant u, \\
\frac{1}{c_{N-n+1}^{\prime}} \frac{\gamma(n)}{\gamma(n-1)} \sum_{\substack{x \in V \\
z \in W}} v\left(\left(\mathrm{e}^{-\nabla_{x z} H_{\Lambda}}-1\right) \mathbb{1}_{E_{x z}}, f^{2} \mid n\right) & \text { otherwise. }\end{cases}
\end{gathered}
$$

With this definition, and using

$$
(\sqrt{x}-\sqrt{y})^{2}=\left(\frac{x-y}{\sqrt{x}+\sqrt{y}}\right)^{2} \leqslant \frac{(x-y)^{2}}{x \vee y},
$$

we have immediately the following corollary

COROLlary 3.7. - In the same setting of Proposition 3.6

$$
\sum_{n} \gamma(n) \wedge \gamma(n-1)\left(\sqrt{v\left(f^{2} \mid n\right)}-\sqrt{v\left(f^{2} \mid n-1\right)}\right)^{2} \leqslant 2 \sum_{n} \gamma_{f}(n)\left(A(n)^{2}+B(n)^{2}\right),
$$

where $\gamma_{f}(n):=\frac{\gamma(n) \wedge \gamma(n-1)}{v\left(f^{2} \mid n\right) \vee v\left(f^{2} \mid n-1\right)}$. 


\subsection{Bound on $\sum_{n} \gamma_{f}(n) A(n)^{2}$}

Here we show how to bound from above the term $\sum_{n} \gamma_{f}(n) A(n)^{2}$ of Corollary 3.7.

PROPOSITION 3.8.-

$$
\sum_{n} \gamma_{f}(n) A(n)^{2} \leqslant k \frac{L^{2}}{\rho_{j}\left|\Lambda_{j}\right|} \mathcal{E}_{v}(f, f) .
$$

Proof. - Let $u=\left\lfloor\rho_{j}|V|\right\rfloor$ and assume, without loss of generality, $\rho_{j} \leqslant \frac{1}{2}$ and $n \leqslant u$. Then we observe that, because of the conservation law,

$$
\frac{1}{c_{n}} \frac{\gamma(n-1)}{\gamma(n)} \sum_{\substack{x \in V \\ z \in W}} v\left(\mathrm{e}^{-\nabla_{x z} H_{\Lambda}} \mathbb{1}_{E_{z x}} \mid n-1\right)=1,
$$

so that

$$
\mathrm{e}^{-2\|\Phi\|} \frac{1}{c_{N_{j}-n+1}^{\prime}} \leqslant \frac{1}{c_{n}} \frac{\gamma(n-1)}{\gamma(n)} \leqslant \mathrm{e}^{2\|\Phi\|} \frac{1}{c_{N_{j-n+1}}^{\prime}} .
$$

Now, using twice the Cauchy-Schwarz inequality together with the identity $a^{2}-b^{2}=$ $(a-b)(a+b)$ on $\nabla_{x z} f^{2}$ gives

$$
\begin{aligned}
A(n)^{2}= & {\left[\frac{1}{c_{n}} \frac{\gamma(n-1)}{\gamma(n)} \sum_{(x, z) \in V \times W} v\left(\mathrm{e}^{-\nabla_{x z} H} \nabla_{x z} f^{2} \mid n-1, E_{z x}\right) v\left(E_{z x} \mid n-1\right)\right]^{2} } \\
\leqslant & k \frac{1}{c_{N_{j}-n+1}^{\prime}} \sum_{(x, z) \in V \times W} v\left(\left(\nabla_{x z} f\right)^{2} \mid n-1, E_{z x}\right) v\left(E_{z x} \mid n-1\right) \\
& \times \frac{1}{c_{n}} \frac{\gamma(n-1)}{\gamma(n)} \sum_{(x, z) \in V \times W} v\left(f^{2}+\left(T_{x z} f\right)^{2} \mid n-1, E_{z x}\right) v\left(E_{z x} \mid n-1\right) .
\end{aligned}
$$

We recall that $v(\cdot \mid n-1)$-almost surely, $\sum_{\substack{x \in W \\ z \in W}} \mathbb{1}_{E_{z x}}=c_{N-n+1}^{\prime}$. So, one can see that

$$
\begin{aligned}
& \frac{1}{c_{n}} \frac{\gamma(n-1)}{\gamma(n)} \sum_{(x, z) \in V \times W} v\left(f^{2} \mid n-1, E_{z x}\right) v\left(E_{z x} \mid n-1\right) \\
& \quad \leqslant k \frac{1}{c_{N_{j}-n+1}^{\prime}} \sum_{(x, z) \in V \times W} v\left(f^{2} \mathbb{1}_{E_{z x}} \mid n-1\right) \\
& \quad=k v\left(f^{2} \mid n-1\right) .
\end{aligned}
$$

On the other hand, using the change of variable $\sigma \mapsto \sigma^{x z}$ and the equality $v(f g)=$ $v(f, g)+v(f) v(g)$, we get

$$
\begin{aligned}
& \frac{1}{c_{n}} \frac{\gamma(n-1)}{\gamma(n)} \sum_{(x, z) \in V \times W} v\left(\left(T_{x z} f\right)^{2} \mid n-1, E_{z x}\right) v\left(E_{z x} \mid n-1\right) \\
& \quad \leqslant \frac{1}{c_{n}} \frac{\gamma(n-1)}{\gamma(n)} \sum_{(x, z) \in V \times W} v\left(\left(T_{x z} f\right)^{2} \mathbb{1}_{E_{z x}} \mid n-1\right)
\end{aligned}
$$




$$
\begin{aligned}
& \leqslant \frac{1}{c_{n}} \sum_{(x, z) \in V \times W} v\left(f^{2} \mathbb{1}_{E_{x z}} \mathrm{e}^{-\nabla_{z x} H} \mid n\right) \\
& \leqslant k v\left(f^{2} \mid n\right),
\end{aligned}
$$

where for the last inequality, we exploited the fact that $v(\cdot \mid n)$-almost surely $\sum_{\substack{x \in V \\ z \in W}} \mathbb{1}_{E_{x z}}=c_{n}$.

Now, since $c_{n}^{\prime}$ is increasing in $n$, we obtain from (3.10), (3.11) and (3.12) that

$$
A(n)^{2} \leqslant k \frac{1}{c_{N_{j}-u+1}^{\prime}}\left\{v\left(f^{2} \mid n\right) \vee v\left(f^{2} \mid n-1\right)\right\} \sum_{(x, z) \in V \times W} v\left(\left(\nabla_{x z} f\right)^{2} \mid n-1\right),
$$

and similarily for $n>u$. Notice that $c_{N_{j}-u+1}^{\prime} \geqslant c_{u}=k \rho_{j}\left|\Lambda_{j}\right|^{2}$. Thus, it follows from (3.13) that

$$
\sum_{n} \gamma_{f}(n) A(n)^{2} \leqslant k \frac{1}{\rho_{j}\left|\Lambda_{j}\right|^{2}} \sum_{(x, z) \in V \times W} v\left(\left(\nabla_{x z} f\right)^{2}\right) \leqslant k \frac{L^{2}}{\rho_{j}\left|\Lambda_{j}\right|} \mathcal{E}_{v}(f, f)
$$

because

$$
\sum_{(x, z) \in V \times W} v\left(\left(\nabla_{x z} f\right)^{2}\right) \leqslant k L^{d+2} \mathcal{E}_{v}(f, f) .
$$

In order to prove (3.15) we need the following definition.

DEFINITION 3.9. - Given a finite connected subset $\Lambda$ of $\mathbb{Z}^{d}$, a path choice in $\Lambda$ is a collection $\lambda=\left\{\lambda_{x z}:(x, z) \in \Lambda \times \Lambda\right\}$ such that $\lambda_{x z}$ is a self-avoiding path from $x$ to $z$ inside $\Lambda$.

Given a path choice $\lambda$ in $\Lambda_{j}$ and $V, W$ as above, we let

$$
\begin{aligned}
& \mathcal{G}_{V}(\lambda)=\max _{e \in \mathcal{E}_{\Lambda}} \#\left\{(x, z) \in V \times W: \lambda_{x z} \sqsupset e\right\}, \\
& \mathcal{D}_{V}(\lambda)=\max _{(x, z) \in V \times W}\left|\lambda_{x z}\right| .
\end{aligned}
$$

Let now $\lambda$ be any path choice. Thanks to Lemma 4.3 in [19] we get that

$$
v\left(\left(\nabla_{x z} f\right)^{2}\right) \leqslant k\left|\lambda_{x z}\right| \sum_{e \sqsubset \lambda_{x z}} v\left(\left(\nabla_{e} f\right)^{2}\right)
$$

which, together with the definition of $\mathcal{G}_{V}(\lambda)$ and $\mathcal{D}_{V}(\lambda)$, implies

$$
\sum_{(x, z) \in V \times W} v\left(\left(\nabla_{x z} f\right)^{2}\right) \leqslant k \mathcal{G}_{V}(\lambda) \mathcal{D}_{V}(\lambda) \mathcal{E}_{v}(f, f) .
$$

A sensible path choice is the following. Given $x \in V$ and $z \in W$, start increasing (or decreasing) the first coordinate of $x$ until it is equal to the first coordinate of $z$. Repeat for the remaining coordinates until you get to $z$. With this particular path choice it is easy to see that $\mathcal{G}_{V}(\lambda) \mathcal{D}_{V}(\lambda) \leqslant k L^{d+2}$ (see Section 5.2 in [10]) and the proof of (3.15) follows at once. 


\subsection{Bound on $\sum_{n} \gamma_{f}(n) B(n)^{2}$}

Here we show how to bound from above the term $\sum_{n} \gamma_{f}(n) B(n)^{2}$ of Corollary 3.7.

PRoposition 3.10. - Assume property $\operatorname{USMT}(C, m, l)$. Then for any $\varepsilon>0$ and $\rho_{0} \leqslant \frac{1}{2}$ there exists $C_{\varepsilon}$ such that, for any $f$ with $v\left(f^{2}\right)=1$,

(i) if $\rho_{j} \leqslant \rho_{0}$

$$
\sum_{n} \gamma_{f}(n) B(n)^{2} \leqslant \frac{1}{\rho_{j}\left|\Lambda_{j}\right|}\left[C_{\varepsilon}+\varepsilon \operatorname{Ent}_{v}\left(f^{2}\right)\right]
$$

(ii) if $\rho_{0} \leqslant \rho_{j} \leqslant \frac{1}{2}$

$$
\sum_{n} \gamma_{f}(n) B(n)^{2} \leqslant \frac{1}{\rho_{j}^{2}\left|\Lambda_{j}\right|}\left[C_{\varepsilon}+C_{\varepsilon} L^{2} \mathcal{E}_{v}(f, f)+\varepsilon \operatorname{Ent}_{v}\left(f^{2}\right)\right] .
$$

Proof. -

(i) Let $u=\left\lfloor\rho_{j}|V|\right\rfloor$ and assume, without loss of generality, $n \leqslant u$. Then, by proceeding as in the proof of Proposition 3.8,

$$
B(n)^{2} \leqslant k v\left(f^{2}, \frac{1}{c_{N_{j}-u+1}^{\prime}} \sum_{\substack{x \in V \\ z \in W}}\left(\mathrm{e}^{-\nabla_{x z} H_{\Lambda}}-1\right) \mathbb{1}_{E_{z x}} \mid n-1\right)^{2},
$$

where $c_{N_{j}-u+1}^{\prime}=\left(N_{j}-u+1\right)(|V|-u+1) \geqslant k \rho_{j}|V||W|$.

Let now $h_{x}(\sigma):=\left(\mathrm{e}^{-\nabla_{x} H_{\Lambda}(\sigma)}-1\right)(1-\sigma(x))$ and $g_{x}(\sigma):=\left(\mathrm{e}^{-\nabla_{x} H_{\Lambda}(\sigma)}-1\right) \sigma(x)$. Then

$$
\begin{aligned}
B(n)^{2} \leqslant & k v\left(f^{2}, \frac{1}{\rho_{j}|V||W|} \sum_{\substack{x \in W \\
z \in W}}\left(\mathrm{e}^{-\nabla_{x z} H_{\Lambda}}-1\right) \mathbb{1}_{E_{z x}} \mid n-1\right)^{2} \\
\leqslant & k v\left(f^{2}, \frac{1}{\rho_{j}|V||W|} \sum_{\substack{x \in V \\
z \in W}}\left[\left(\mathrm{e}^{-\nabla_{x z} H_{\Lambda}}-1\right) \mathbb{1}_{E_{z x}}-h_{x} g_{z}\right] \mid n-1\right)^{2} \\
& +k v\left(f^{2}, \frac{1}{\rho_{j}|V||W|} \sum_{\substack{x \in V \\
z \in W}} h_{x} g_{z} \mid n-1\right)^{2}
\end{aligned}
$$

Notice that

$$
\left(\mathrm{e}^{-\nabla_{x z} H_{\Lambda}}-1\right) \mathbb{1}_{E_{z x}}-g_{z} h_{x}=g_{z}\left(1-\sigma_{x}\right)+h_{x} \sigma_{z} \quad \text { if } d(x, z)>r .
$$

Thus the first term in the r.h.s. of (3.17) can be bounded from above by

$$
\begin{aligned}
& k v\left(f^{2}, \frac{1}{\rho_{j}|V||W|} \sum_{\substack{x, z) \in V \times W \\
d(x, z) \leqslant r}}\left[\left(\mathrm{e}^{-\nabla_{x z} H_{\Lambda}}-1\right) \mathbb{1}_{E_{z x}}-h_{x} g_{z}\right] \mid n-1\right)^{2} \\
& \quad+k v\left(f^{2}, \frac{|V|-n+1}{\rho_{j}|W||V|} \sum_{z \in W} g_{z} \mid n-1\right)^{2}
\end{aligned}
$$




$$
+k v\left(f^{2}, \frac{N_{j}-n+1}{\rho_{j}|W||V|} \sum_{x \in V} h_{x} \mid n-1\right)^{2} .
$$

In order to bound the second and third term in (3.18) we can apply Proposition 6.2 to the functions $\sum_{z \in W} g_{z}$ and $\sum_{x \in V} \rho_{j} h_{x}$. The result is that the sum of second and third term in the r.h.s. of (3.18) is smaller than

$$
k \frac{1}{\rho_{j}\left|\Lambda_{j}\right|} v\left(f^{2} \mid n-1\right)\left[C_{\varepsilon} v\left(f^{2} \mid n-1\right)+\varepsilon \operatorname{Ent}_{v(\cdot \mid n-1)}\left(f^{2}\right)\right] .
$$

Notice that, if we average (3.19) over $\gamma_{f}(n)$ we get, after a Jensen inequality, the sought bound. Finally we consider the first term in the r.h.s. of (3.18). A simple $L^{\infty}$ bound gives that this term is smaller than

$$
k \frac{1}{L^{2}} \frac{1}{\rho_{j}^{2}\left|\Lambda_{j}\right|^{2}} v\left(f^{2} \mid n-1\right)^{2}
$$

and thus, by recalling the definition of $\gamma_{f}(n)$, the result is obtained. To bound the second term in (3.17) one has to use point (i) of Proposition 6.5.

(ii) We can proceed as in the previous case using Proposition 6.3 instead of Proposition 6.2 and point (ii) of Proposition 6.5 instead of point (i).

\subsection{On the distribution of the number of particles inside one block}

The aim of this paragraph is first to recall some general result on the distribution of the number of particles inside one block $\Lambda_{j}$ and secondly to derive an (optimal) upper bound on its logarithmic Sobolev constant (see Proposition 3.17 below).

The setting is that of Section 3.4 above. We assume without loss of generality $\rho_{j} \leqslant \frac{1}{2}$ and we denote by $\bar{n}=\mu\left(N_{V}\right)$.

We also set $n_{\min }=\max \left\{0, N_{j}-|W|\right\}$ and $n_{\max }=\min \left\{|V|, N_{j}\right\}$ to be the smallest and the largest value of $N_{V}(\sigma)$ under the constraint that $N_{\Lambda_{j}}(\sigma)=N_{j}$. It is easy to check that $\frac{1}{k} \bar{n} \leqslant n_{\max }-\bar{n} \leqslant k \bar{n}$ and similarly for $\bar{n}-n_{\min }$.

In what follows we will consider the distribution of the number of particles in $V$ under the measure $v$. More precisely we define $\gamma=\{\gamma(n)\}$ to be the probability measure on $I=\left\{n \in\left[n_{\min }, n_{\max }\right]: n\right.$ is an integer $\}$, given by

$$
\gamma(n):=v\left(N_{V}=n\right) .
$$

In order to obtain sharp bounds on $\gamma(n)$, we modify the chemical potential $\underline{\lambda}$ appearing in $\mu:=\mu_{\Lambda}^{\tau, \underline{\lambda}}$ in an $n$-dependent way in such a way that the value $n$ becomes the new average value of $N_{V}$ under $\mu_{\Lambda}^{\tau, \underline{\lambda}}$. More precisely, given $n \in\left[n_{\min }, n_{\max }\right]$, let $\underline{\lambda}(n)=$ $\left\{\lambda_{1}, \ldots, \lambda_{j-1}, \lambda_{V}, \lambda_{W}, \lambda_{j+1}, \ldots, \lambda_{j_{\max }}\right\}$ be a new chemical potential constant on the atoms of the new partition and such that

$$
\begin{aligned}
& \mu_{\Lambda}^{\tau, \underline{\lambda}(n)}\left(N_{\Lambda_{i}}\right)=N_{i}, \quad i=1, \ldots, j_{\max }, \\
& \mu_{\Lambda}^{\tau, \underline{\lambda}(n)}\left(N_{V}\right)=n, \\
& \mu_{\Lambda}^{\tau, \underline{\lambda}(n)}\left(N_{W}\right)=N_{j}-n .
\end{aligned}
$$


It is then easy to check that $\gamma$ can be written in the Gibbsian form, $\gamma(n)=\mathrm{e}^{-H(n)} \varphi(n)$, where

$$
\begin{aligned}
H(n) & :=\sum_{i \neq j}\left(\lambda_{i}(n)-\lambda_{i}\right) N_{i}+\lambda_{V} n+\lambda_{W}\left(N_{j}-n\right)-\lambda_{j} N_{j}-\log \left(\frac{Z_{\Lambda}^{\tau, \underline{\lambda}(n)}}{Z_{\Lambda}^{\tau, \underline{\lambda}}}\right), \\
\varphi(n) & :=\frac{\mu_{\Lambda}^{\tau, \underline{\lambda}(n)}\left(\mathbf{N}_{\Lambda}=\mathbf{N} ; N_{V}=n\right)}{\mu_{\Lambda}^{\tau, \underline{\lambda}}\left(\mathbf{N}_{\Lambda}=\mathbf{N}\right)} .
\end{aligned}
$$

Finally, given $\varepsilon \in(0,1)$, we consider for technical reasons the " $\varepsilon$-regularization" ( $\varepsilon$ here must be thougth to be close to one) of $\gamma$ defined by

$$
\gamma_{\varepsilon}(n):= \begin{cases}\frac{1}{Z} \mathrm{e}^{-H(n)} & \text { if } n \in I_{\varepsilon}, \\ \gamma(n) & \text { otherwise }\end{cases}
$$

where

$$
\begin{aligned}
Z & :=\frac{\sum_{n \in I_{\varepsilon}} \mathrm{e}^{-H(n)}}{\sum_{n \in I_{\varepsilon}} \gamma(n)}, \\
n_{\max }^{\varepsilon} & =\bar{n}+\varepsilon\left(n_{\max }-\bar{n}\right), \\
n_{\min }^{\varepsilon} & =\bar{n}+\varepsilon\left(n_{\min }-\bar{n}\right), \\
I_{\varepsilon} & :=\left[n_{\min }^{\varepsilon}, n_{\max }^{\varepsilon}\right] \cap I .
\end{aligned}
$$

We now recall, without any proof, some results on $\gamma_{\varepsilon}$ established in Section 3.4 and 4 of [10] under the mixing hypothesis $\operatorname{USMT}(C, m, l)$ that will turn out to be useful. Then, we complete these properties by some others in order to prove that $\gamma_{\varepsilon}$ satisfies a convex condition. Once convexity is established, we can appeal to Proposition A.5 in the appendix to give a sharp bound on its logarithmic Sobolev constant.

The first result concerns the behaviour of the "potential" $H(n)$. As usual $k$ will denote an arbitrary constant depending only on $C, m, l, \delta, \varepsilon,\|\Phi\|_{\infty}$ and whose value varies from time to time.

LEMMA 3.11. - For all $n \in I_{\varepsilon}$,

$$
\begin{array}{ll}
\frac{1}{k} \frac{n-\bar{n}}{\bar{n}} \leqslant H(n+1)-H(n) \leqslant k \frac{n-\bar{n}}{\bar{n}} & \text { for all } n \geqslant \bar{n}, \\
\frac{1}{k} \frac{\bar{n}-n}{\bar{n}} \leqslant H(n-1)-H(n) \leqslant k \frac{\bar{n}-n}{\bar{n}} & \text { for all } n \leqslant \bar{n} .
\end{array}
$$

The next two results show that the relative density between $\gamma$ and its regularization is bounded uniformly in the size of $I$ and that the tails of $\gamma(n)$ are at least exponential. More precisely

LEMMA 3.12. -

$$
\frac{1}{k} \leqslant \inf _{n \in I} \frac{\gamma(n)}{\gamma_{\varepsilon}(n)} \leqslant \sup _{n \in I} \frac{\gamma(n)}{\gamma_{\varepsilon}(n)} \leqslant k .
$$

LEMMA 3.13. - There exists a positive constant $\varepsilon_{0}=\varepsilon\left(\delta,\|\Phi\|_{\infty}\right)$ such that $\forall \varepsilon \in$ $\left(\varepsilon_{0}, 1\right)$ 


$$
\begin{aligned}
& \frac{\gamma(n+1)}{\gamma(n)} \leqslant \frac{1}{2} \quad \text { if } n \in\left[n_{\max }^{\varepsilon}, n_{\max }\right], \\
& \frac{\gamma(n-1)}{\gamma(n)} \leqslant \frac{1}{2} \quad \text { if } n \in\left[n_{\min }, n_{\min }^{\varepsilon}\right] .
\end{aligned}
$$

COROLlary 3.14.-

$$
\frac{\gamma_{\varepsilon}(n+1)}{\gamma_{\varepsilon}(n)} \leqslant k \mathrm{e}^{-k \frac{n-\bar{n}}{\bar{n}}} \quad \forall n \geqslant \bar{n}
$$

and similarity for $\frac{\gamma_{\varepsilon}(n-1)}{\gamma_{\varepsilon}(n)}, n \leqslant \bar{n}$.

The next result shows that the normalization factor $Z$ appearing in the definition of $\gamma_{\varepsilon}$ is close to $\bar{n}^{1 / 2}$.

LEMMA 3.15. -

$$
\frac{1}{k} \sqrt{\bar{n}} \leqslant Z \leqslant k \sqrt{\bar{n}}
$$

Proof. - We must consider two terms, $\sum_{n \in I_{\varepsilon}} \mathrm{e}^{-H(n)}$ and $\sum_{n \in I_{\varepsilon}} \gamma(n)$.

By using Lemma 3.11 one can easily get for all $n \in I_{\varepsilon}$

$$
\frac{1}{k} \mathrm{e}^{-\frac{k}{n}(\bar{n}-n)^{2}} \leqslant \mathrm{e}^{-H(n)} \leqslant k \mathrm{e}^{-\frac{1}{k \bar{n}}(\bar{n}-n)^{2}} .
$$

Thus, using a comparison with integrals and a change of variable, it easily follows that

$$
\frac{1}{k} \sqrt{\bar{n}} \leqslant \sum_{n \in I_{\varepsilon}} \mathrm{e}^{-H(n)} \leqslant k \sqrt{\bar{n}}
$$

On the other hand, it follows from Lemma 3.12 that

$$
\begin{aligned}
\sum_{n=n_{\min }}^{n_{\min }^{\varepsilon}} \gamma(n) & =\gamma\left(n_{\min }^{\varepsilon}\right) \sum_{n=n_{\min }}^{n_{\min }^{\varepsilon}} \frac{\gamma(n)}{\gamma\left(n_{\min }^{\varepsilon}\right)} \\
& =\gamma\left(n_{\min }^{\varepsilon}\right) \sum_{n=n_{\min }}^{n_{\min }} \prod_{j=n+1}^{n_{\min }^{\varepsilon}} \frac{\gamma(j-1)}{\gamma(j)} \\
& \leqslant \gamma\left(n_{\min }^{\varepsilon}\right) \sum_{n=n_{\min }}^{n_{\min }^{\varepsilon}}\left(\frac{1}{2}\right)^{n_{\min }^{\varepsilon}-n} \\
& \leqslant 2 \gamma\left(n_{\min }^{\varepsilon}\right) .
\end{aligned}
$$

A similar statement for $\sum_{n=n_{\max }^{\varepsilon}}^{n_{\max }} \gamma(n)$ yields

$$
\sum_{n \in I_{\varepsilon}} \gamma(n) \geqslant 1-2\left(\gamma\left(n_{\min }^{\varepsilon}\right)+\gamma\left(n_{\max }^{\varepsilon}\right)\right) .
$$


We are left with the problem of bounding from above $\gamma\left(n_{\min }^{\varepsilon}\right)$ and $\gamma\left(n_{\max }^{\varepsilon}\right)$. Because of Lemma 3.13 it's enough to control $\gamma_{\varepsilon}\left(n_{\min }^{\varepsilon}\right)$ and $\gamma_{\varepsilon}\left(n_{\max }^{\varepsilon}\right)$. By using Lemma 3.11 together with Corollary 3.14, we get

$$
\begin{aligned}
\gamma_{\varepsilon}\left(n_{\min }^{\varepsilon}\right) & =\gamma_{\varepsilon}(\bar{n}) \prod_{j=n_{\min }^{\varepsilon}+1}^{\bar{n}} \frac{\gamma_{\varepsilon}(j-1)}{\gamma_{\varepsilon}(j)} \\
& \leqslant k \mathrm{e}^{-\frac{k}{2 n}\left(\bar{n}-n_{\min }^{\varepsilon}\right)^{2}} \\
& \leqslant k \mathrm{e}^{-\frac{k}{n}\left(n_{\min }-\bar{n}\right)^{2}} \\
& \leqslant k \mathrm{e}^{-k\left(\bar{n}-n_{\min }\right)} .
\end{aligned}
$$

In conclusion, we get that $\gamma\left(n_{\min }^{\varepsilon}\right) \leqslant \frac{1}{8}$ provided that $\bar{n}$ is large enough. A similar statement for $\gamma\left(n_{\max }^{\varepsilon}\right)$ gives finally that

$$
1 \geqslant \sum_{n \in I_{\varepsilon}} \gamma(n) \geqslant \frac{1}{2}
$$

This result together with (3.25) completes the proof.

From Lemmas 3.11 and 3.15, one can easily see that $\gamma_{\varepsilon}$ is bell shaped around $\bar{n}$ at least if we restrict it to the interval $I_{\varepsilon}$. Our last result shows that, in some sense, this property holds also outside $I_{\varepsilon}$.

LEMMA 3.16. -

$$
\frac{1}{k \sqrt{\bar{n}}} \mathrm{e}^{-\frac{k}{n}(\bar{n}-n)^{2}} \leqslant \gamma_{\varepsilon}(n) \leqslant \frac{k}{\sqrt{\bar{n}}} \mathrm{e}^{-\frac{1}{k \bar{n}}(\bar{n}-n)^{2}} .
$$

Proof. - Thanks to Lemmas 3.11, 3.15 and the fact that $n_{\max }-\bar{n}$ is proportional to $\bar{n}$ one can see that (3.26) holds for all $n \in I_{\varepsilon}$. Moreover, by using Corollary 3.14 the upper bound of (3.26) holds for all $n \in I$. It is therefore enough to prove the lower bound of (3.26) for $n \in I \backslash I_{\varepsilon}$.

For this purpose, we let $n \in\left[n_{\max }^{\varepsilon}, n_{\max }\right]$ (the case $n \in\left[n_{\min }, n_{\min }^{\varepsilon}\right]$ being similary) and we recall that, thanks to Proposition 3.6 of [10],

$$
k \frac{(|V|-n)\left(N_{j}-n\right)}{(n+1)\left(|W|-N_{j}+n+1\right)} \leqslant \frac{\gamma(n+1)}{\gamma(n)} .
$$

Therefore

$$
\begin{aligned}
\gamma(n) & \geqslant\left(n_{\max }^{\varepsilon}\right) \mathrm{e}^{-k\left(n-n_{\max }^{\varepsilon}\right)} \prod_{n_{\max }^{\varepsilon}}^{n-1} \frac{(|V|-i)\left(N_{j}-i\right)}{(i+1)\left(|W|-N_{j}+i+1\right)} \\
& \geqslant \frac{k}{\sqrt{\bar{n}}} \mathrm{e}^{-\frac{1}{k \bar{n}}(\bar{n}-n)^{2}},
\end{aligned}
$$

where we have used (3.26) to get the exponential bound for $\gamma\left(\eta_{\max }^{\varepsilon}\right)$, Stierling formula and a straightforward calculation to bound from below the product. The proof is complete. 
We are finally in a position to state the main result of this paragraph.

Proposition 3.17. - Assume property $\operatorname{USMT}(C, m, l)$. Then, for all $f: \Omega_{\Lambda} \mapsto \mathbb{R}$ that depend only on $N_{V}(\sigma)$, the following logarithmic Sobolev inequality holds

$$
\operatorname{Ent}_{\gamma}(f) \leqslant k \bar{n} \sum_{n \in I}(\gamma(n) \wedge \gamma(n-1))[f(n)-f(n-1)]^{2} \text {. }
$$

Proof.-Because of Lemma 3.12 it is sufficient to prove the logarithmic Sobolev inequality for the regularized measure $\gamma_{\varepsilon}$.

Pick $\varepsilon$ sufficiently close to one in such a way that Lemma 3.13 holds. Then, thanks to Corollary 3.14 and Lemma 3.16, the regularized measure $\gamma_{\varepsilon}$ satisfies the convex hypothesis $\operatorname{CONV}(c, \bar{n})$ described in the appendix, for some $c$ independent of $\bar{n}$ and $I$. Therefore we can apply Proposition A.5 in the appendix and get the result.

\section{Recursive estimate of the logarithmic Sobolev constant}

In this section we prove the main result of the paper, Theorem 2.3 , via a recursive analysis on the behaviour of the logarithmic Sobolev constant when the linear size of the volume under consideration is doubled. For simplicity we carry out our analysis in two dimensions but the extension to higher dimension is straightforward (see remark at the end of Section 3.3). We confine ourselves with the proof of the upper bound since the lower bound is easily proved by plugging a suitable test function (a slowly varying function of the local density) inside the logarithmic Sobolev inequality (2.11).

DEFINITION. $-\mathcal{R}_{L}$ will denote the class of rectangles in $\mathbb{Z}^{2}$, which, modulo translations and permutations of the coordinates, can be written as $R\left(l_{1}, l_{2}\right)=\left[0, l_{1}-1\right] \times$ $\left[0, l_{2}-1\right]$ with $0.1 l_{2} \leqslant l_{1} \leqslant l_{2} \leqslant L$. We also set

$$
c_{S}(L)=\max _{\mathcal{R} \in \mathcal{R}_{L}} \max _{N, \tau} c_{R, N}^{\tau},
$$

where $c_{R, N}^{\tau}$, the logarithmic Sobolev constant in $R$ with boundary condition $\tau$ and $N$ particles, has been defined in (2.11).

With the above notation we will prove the following recursive bound.

THEOREM 4.1. - Assume $\operatorname{USMT}(C, m, l)$. Then there exists a positive constant $k=k(d, r,\|\Phi\|)$ such that

$$
c_{s}(2 L) \leqslant \frac{3}{2} c_{s}(L)+k L^{2}
$$

for all L large enough. In particular $\sup _{L \geqslant l} L^{-2} c_{s}(L)<+\infty$.

Proof. - Let us consider a rectangle $\Lambda:=R\left(l_{1}, l_{2}\right) \in \mathcal{R}_{2 L} \backslash \mathcal{R}_{L}$ with $l_{1} \leqslant l_{2}$ and let us fix a small number $\delta_{0} \in\left(0,10^{-2}\right)$ and we set $d=\left\lfloor\delta_{0} L\right\rfloor$. Given an integer 
$i \in\left[1,\left\lfloor\frac{1}{10 \delta_{0}}\right\rfloor-1\right]$, we partition $\Lambda$ into four disjoint sub-rectangles $\left\{\Lambda_{j}\right\}_{j=1}^{4}$ as follows

$$
\begin{aligned}
& \Lambda_{1}=\left\{x \in \Lambda ; 0 \leqslant x_{2} \leqslant l_{2} / 2+(i-1) d\right\}, \\
& \Lambda_{2}=\left\{x \in \Lambda ;(i-1) d<x_{2} \leqslant i d\right\}, \\
& \Lambda_{3}=\left\{x \in \Lambda ; i d<x_{2} \leqslant(i+1) d\right\}, \\
& \Lambda_{4}=\left\{x \in \Lambda ;(i+1) d<x_{2}\right\},
\end{aligned}
$$

and we set $A=\Lambda_{1} \cup \Lambda_{2} \cup \Lambda_{3}, B=\Lambda_{2} \cup \Lambda_{3} \cup \Lambda_{4}, S=\Lambda_{2} \cup \Lambda_{3}$.

Fix now a boundary condition $\tau$ outside $\Lambda$, a number of particles $N \in[0, \ldots,|\Lambda|]$ and let $v:=v_{\Lambda, N}^{\tau}$. We will then use three times the formula relating the entropy of $f^{2}$ w.r.t. the measure $v, \operatorname{Ent}_{v}\left(f^{2}\right)$, to the entropy of $f^{2}$ w.r.t. the measure $v\left(\cdot \mid \mathcal{F}_{0}\right)$ conditioned to a sub $\sigma$-algebra $\mathcal{F}_{0}, \operatorname{Ent}_{v}\left(f^{2} \mid \mathcal{F}_{0}\right)$ :

$$
\operatorname{Ent}_{v}\left(f^{2}\right)=v\left(\operatorname{Ent}_{v}\left(f^{2} \mid \mathcal{F}_{0}\right)\right)+\operatorname{Ent}_{v}\left(v\left[f^{2} \mid \mathcal{F}_{0}\right]\right)
$$

to write

$$
\begin{aligned}
\operatorname{Ent}_{v}\left(f^{2}\right)= & v\left(\operatorname{Ent}_{v}\left(f^{2} \mid N_{A}\right)\right)+\operatorname{Ent}_{v}\left(v\left[f^{2} \mid N_{A}\right]\right) \\
= & v\left(\operatorname{Ent}_{v}\left(f^{2} \mid N_{A}, N_{S}\right)\right)+v\left(\operatorname{Ent}_{v}\left(v\left[f^{2} \mid N_{A}, N_{S}\right] \mid N_{A}\right)\right)+\operatorname{Ent}_{v}\left(v\left[f^{2} \mid N_{A}\right]\right) \\
= & v\left(\operatorname{Ent}_{v}\left(f^{2} \mid N_{\Lambda_{1}}, N_{\Lambda_{2}}, N_{\Lambda_{3}}\right)\right)+v\left(\operatorname{Ent}_{v}\left(v\left[f^{2} \mid N_{\Lambda_{1}}, N_{\Lambda_{2}}, N_{\Lambda_{3}}\right] \mid N_{A}, N_{S}\right)\right) \\
& +v\left(\operatorname{Ent}_{v}\left(v\left[f^{2} \mid N_{A}, N_{S}\right] \mid N_{A}\right)\right)+\operatorname{Ent}_{v}\left(v\left[f^{2} \mid N_{A}\right]\right),
\end{aligned}
$$

where, we recall, $N_{V}$ denotes the number of particles in the region $V$. Formula (4.3) will represent our basic starting point. We will now examine separately each term in the r.h.s. of (4.3).

As usual, in what follows, $k$ will denote a generic constant depending on the interaction, on the dimensions of the lattice and on $\delta_{0}$, whose value may vary from line to line.

\subsection{Analysis of the first term in the r.h.s. of (4.3)}

For any small $\varepsilon$ and large enough $L$, we can use Proposition 3.5 to bound from above the first term in the r.h.s. of (4.3) by

$$
\begin{aligned}
v\left(\operatorname{Ent}_{v}\left(f^{2} \mid N_{\Lambda_{1}}, N_{\Lambda_{2}}, N_{\Lambda_{3}}\right)\right) & \leqslant v\left(\operatorname{Ent}_{v_{A}}\left(f^{2}\right)+\operatorname{Ent}_{v_{B}}\left(f^{2}\right)\right)+(2+\varepsilon) \operatorname{Var}_{v}(f) \\
& \leqslant v\left(\operatorname{Ent}_{v_{A}}\left(f^{2}\right)+\operatorname{Ent}_{v_{B}}\left(f^{2}\right)\right)+k L^{2} \mathcal{E}_{v}(f, f),
\end{aligned}
$$

where the average is over the number of particles in $A(B)$ and over the boundary conditions outside $A,(B)$. Above we have used the trivial bound $v\left(\operatorname{Var}_{v}\left(f \mid N_{A}, N_{\Lambda_{2}}, N_{\Lambda_{3}}\right)\right) \leqslant$ $\operatorname{Var}_{v}(f)$ together with (3.6) and the spectral gap estimate (see [14] and [10])

$$
\operatorname{Var}_{v}(f) \leqslant k L^{2} \mathcal{E}_{v}(f, f) .
$$

Let us now examine the geometry of the bottom rectangle $A$, the reasoning being similar for the top one. There are two cases to analyze:

(a) $l_{1} \leqslant \frac{3}{2} L$. In this case one easily verifies that $A \in \mathcal{R}_{\frac{3}{2} L}$. 
(b) $l_{1}>\frac{3}{2} L$. In this case $A \in \mathcal{R}_{2 L}$ but now the longest side is $l_{1}$ and the shortest one is smaller than $l_{2} / 2+(i+1) d$ which in turn is smaller than $1.2 L$ by construction. Therefore $\max _{\tau, N} c_{A, N}^{\tau} \leqslant \max \left\{c_{s}\left(\frac{3}{2} L\right), \hat{c}_{s}(2 L)\right\}$ where

$$
\hat{c}_{S}(2 L)=\max _{\substack{R \in \mathcal{R}_{2 L} L \\ l_{1} \leqslant 1.2 L, N \\ l_{2} \geqslant \frac{3}{2} L}} \max _{\tau, N} c_{R, N}^{\tau}
$$

In other words

$$
v\left(\operatorname{Ent}_{v_{A}}\left(f^{2}\right)\right) \leqslant \max \left\{c_{s}\left(\frac{3}{2} L\right), \hat{c}_{s}(2 L)\right\} \mathcal{E}_{v}(f, f)
$$

and similarily for $B$.

In conclusion, we obtain that the r.h.s. of (4.4) is smaller than

$$
\max \left\{c_{s}\left(\frac{3}{2} L\right), \hat{c}_{s}(2 L)\right\}\left[\mathcal{E}_{v}(f, f)+\frac{1}{2} \sum_{[x, y] \in \mathcal{E}_{S}} v\left[c_{x y}\left(\nabla_{x y} f\right)^{2}\right]\right]+k L^{2} \mathcal{E}_{v}(f, f)
$$

uniformly in $i \in\left[1,\left\lfloor\frac{1}{10 \delta_{0}}-1\right\rfloor\right]$. Notice that the "spurious" term $\frac{1}{2} \sum_{[x, y] \in \mathcal{E}_{S}} v\left[c_{x y}\left(\nabla_{x y} f\right)^{2}\right]$ comes from the fact that $A \cap B=S$.

\subsection{Analysis of the remaining terms in the r.h.s. of (4.3)}

Here we bound from above the other three terms in (4.3). The necessary steps are almost identical for all of them and therefore, for shortness, we treat only the second one (the last one enjoys some minor simplifications not shared by the other two). Later on we will state without further comments the analogous result for the first and third one.

For a given value $N_{A}$ of the number of particles in $A$, let $\rho_{A}:=\frac{N_{A}}{|A|}$ and assume, without loss of generality, that $\rho_{A} \leqslant \frac{1}{2}$. Let $\hat{v}(\cdot):=v\left(\cdot \mid N_{A}\right)$ be the associated multicanonical measure and let $\hat{\mu}$ be the corresponding (multi)-grand canonical measure. Let also $N_{S}^{*}=\hat{\mu}\left(N_{S}\right)$, and let $\gamma(n):=\hat{v}\left(N_{S}=n\right)$. Notice that $N_{S}^{*} \leqslant k \rho_{A} L^{d}$ (see Proposition 3.1 of [9]). Then, using Corollary 3.7, we can write

$$
\begin{aligned}
& \operatorname{Ent}_{v}\left(v\left[f^{2} \mid N_{A}, N_{S}\right] \mid N_{A}\right)=\operatorname{Ent}_{\hat{v}}\left(\hat{v}\left[f^{2} \mid N_{S}\right]\right)=\operatorname{Ent}_{\gamma}\left(\hat{v}\left[f^{2} \mid N_{S}\right]\right) \\
& \quad \leqslant k \rho_{A} L^{d} \sum_{n} \gamma(n) \wedge \gamma(n-1)\left(\sqrt{\hat{v}\left(f^{2} \mid N_{S}=n\right)}-\sqrt{\hat{v}\left(f^{2} \mid N_{S}=n-1\right)}\right)^{2} \\
& \quad \leqslant k \rho_{A} L^{d} \sum_{n} \gamma_{f}(n)\left(A(n)^{2}+B(n)^{2}\right),
\end{aligned}
$$

where $A(n)$ and $B(n)$ have been defined in (3.9).

Thanks to Proposition 3.8 and 3.10 , for any $\varepsilon>0$ there exists a constant $C_{\varepsilon}$ independent of $\rho_{A}$ such that the r.h.s. of (4.6) is smaller than

$$
C_{\varepsilon} \hat{v}\left(f^{2}\right)+C_{\varepsilon} L^{2} \mathcal{E}_{\hat{v}}(f, f)+\varepsilon \operatorname{Ent}_{\hat{v}}\left(f^{2}\right) .
$$

Thus, if we average w.r.t. the canonical measure $v$ the 1.h.s. of (4.6) and use the simple inequality $v\left(\operatorname{Ent}_{v}\left(f^{2} \mid \mathcal{F}_{0}\right)\right) \leqslant \operatorname{Ent}_{v}\left(f^{2}\right)$ for any $\mathcal{F}_{0}$, we get 


$$
\begin{aligned}
v\left(\operatorname{Ent}_{v}\left(v\left[f^{2} \mid N_{A}, N_{S}\right] \mid N_{A}\right)\right) & =v\left(\operatorname{Ent}_{\hat{v}}\left(v\left[f^{2} \mid N_{A}, N_{S}\right]\right)\right) \\
& \leqslant C_{\varepsilon} v\left(f^{2}\right)+C_{\varepsilon} L^{2} \mathcal{E}_{v}(f, f)+\varepsilon \operatorname{Ent}_{v}\left(f^{2}\right) .
\end{aligned}
$$

Similar bounds hold also for the first and third term in the r.h.s. of (4.3).

\subsection{The recursion completed}

We are finally in a position to complete the proof of Theorem 4.1. If we put together (4.8) and (4.5) we get that, for any $\varepsilon$ small enough

$$
\begin{aligned}
\text { r.h.s. of }(4.3) \leqslant & \max \left\{c_{s}\left(\frac{3}{2} L\right), \hat{c}_{s}(2 L)\right\}\left[\mathcal{E}_{v}(f, f)+\frac{1}{2} \sum_{[x, y] \in \mathcal{E}_{s}} v\left[c_{x y}\left(\nabla_{x y} f\right)^{2}\right]\right] \\
& +C_{\varepsilon} v\left(f^{2}\right)+C_{\varepsilon} L^{2} \mathcal{E}_{v}(f, f)+\varepsilon \operatorname{Ent}_{v}\left(f^{2}\right),
\end{aligned}
$$

that is

$$
\begin{aligned}
\operatorname{Ent}_{v}\left(f^{2}\right) \leqslant & \left(\frac{1}{1-\varepsilon}\right) \max \left\{c_{s}\left(\frac{3}{2} L\right)^{-1}, \hat{c}_{s}(2 L)^{-1}\right\} \\
& \times\left[\mathcal{E}_{v}(f, f)+\frac{1}{2} \sum_{[x, y] \in \mathcal{E}_{S}} v\left[c_{x y}\left(\nabla_{x y} f\right)^{2}\right]\right] \\
& +C_{\varepsilon} v\left(f^{2}\right)+C_{\varepsilon} L^{2} \mathcal{E}_{v}(f, f)
\end{aligned}
$$

for a suitable constant $C_{\varepsilon}$.

Finally, following [16], we average the above inequality w.r.t. to the integer $i$ (see (4.1)) and use the observation that, as $i$ varies in $\left[1, \frac{1}{10 \delta_{0}}\right]$, the strips $S \equiv S_{i}$ are disjoint. In particular

$$
\frac{1}{2} \sum_{i \in\left[1, \frac{1}{10 \delta_{0}}\right]} \sum_{x, y] \in \mathcal{E}_{S_{i}}} v\left[c_{x y}\left(\nabla_{x y} f\right)^{2}\right] \leqslant \mathcal{E}_{v}(f, f)
$$

so that

$$
\begin{aligned}
\operatorname{Ent}_{v}\left(f^{2}\right) \leqslant & \left(\frac{1}{1-2 \varepsilon}\right)\left(1+\left\lfloor 10 \delta_{0}\right\rfloor\right) \max \left\{c_{s}\left(\frac{3}{2} L\right), \hat{c}_{s}(2 L)\right\} \mathcal{E}_{v}(f, f) \\
& +C_{\varepsilon} v\left(f^{2}\right)+C_{\varepsilon} L^{2} \mathcal{E}_{v}(f, f) .
\end{aligned}
$$

Notice that if we write $f=[f-v(f)]+v(f)$ and we use the Poincaré bound $\operatorname{Var}_{v}(f) \leqslant k L^{2} \mathcal{E}_{v}(f, f)$ we get

$$
\begin{aligned}
\operatorname{Ent}_{v}\left(f^{2}\right) \leqslant & \operatorname{Ent}_{v}\left([f-v(f)]^{2}\right)+2 \operatorname{Var}_{v}(f) \\
\leqslant & \left(\frac{1}{1-2 \varepsilon}\right)\left(1+\left\lfloor 10 \delta_{0}\right\rfloor\right) \max \left\{c_{s}\left(\frac{3}{2} L\right), \hat{c}_{s}(2 L)\right\} \mathcal{E}_{v}(f, f) \\
& +k C_{\varepsilon} L^{2} \mathcal{E}_{v}(f, f),
\end{aligned}
$$

where in the first line we have used once more the Rothaus inequality (see [2]).

In other words

$$
c_{\Lambda, N}^{\tau} \leqslant\left(\frac{1}{1-2 \varepsilon}\right)\left(1+\left\lfloor 10 \delta_{0}\right\rfloor\right) \max \left\{c_{s}\left(\frac{3}{2} L\right), \hat{c}_{s}(2 L)\right\}+k C_{\varepsilon} L^{2} .
$$


Notice that if the original rectangle $\Lambda$ was such that $l_{1} \leqslant 1.2 L$ while $l_{2} \geqslant \frac{3}{2} L$, i.e. $\Lambda$ was chosen in the sub-class of $\mathcal{R}_{2 L}$ entering in the definition of $\hat{c}_{S}(2 L)$, then we would have obtained the inequality (4.13) with the factor $\max \left\{c_{s}\left(\frac{3}{2} L\right), \hat{c}_{s}(2 L)\right\}$ replaced by $c_{s}\left(\frac{3}{2} L\right)$ simply because case $b$ ) right after (4.4) would have been impossible. Thus

$$
\hat{c}_{s}(2 L) \leqslant\left(\frac{1}{1-2 \varepsilon}\right)\left(1+\left\lfloor 10 \delta_{0}\right\rfloor\right) c_{s}\left(\frac{3}{2} L\right)+k C_{\varepsilon} L^{2} .
$$

If we combine (4.13) with (4.14) we finally get

$$
c_{\Lambda, N}^{\tau} \leqslant\left(\frac{1}{1-2 \varepsilon}\right)^{2}\left(1+\left\lfloor 10 \delta_{0}\right\rfloor\right)^{2} c_{s}\left(\frac{3}{2} L\right)+C_{\varepsilon}^{\prime} L^{2}
$$

for another constant $C_{\varepsilon}^{\prime}$. Thus

$$
c_{s}(2 L) \leqslant\left(\frac{1}{1-2 \varepsilon}\right)^{2}\left(1+\left\lfloor 10 \delta_{0}\right\rfloor\right)^{2} c_{s}\left(\frac{3}{2} L\right)+C_{\varepsilon}^{\prime} L^{2}
$$

and two more iterations prove the recursive inequality of the theorem provided that the two parameters $\varepsilon, \delta_{0}$ were chosen small enough.

Finally the fact that $\max _{L}\left(c_{s}(L) L^{-2}\right)>0$ is a trivial consequence of the recursive bound.

\section{On the grand canonical Laplace transform}

In this section we seek Gaussian bounds on quantities of the form $\mu\left(\mathrm{e}^{t f}\right)$ where $\mu$ is the grand canonical Gibbs measure on some finite set and $f$ is a mean zero function, namely bounds of the type

$$
\mu\left(\mathrm{e}^{t f}\right) \leqslant \mathrm{e}^{t^{2} K_{f}} .
$$

Once bounds like the one above are proved, then we can transfer them to the canonical Laplace transform by means of Proposition 3.3.

We first explain in some general terms our approach based on a combination of the so-called Herbst's approach and the DLR equation and then we consider some concrete cases that will play a key role in the recursive analysis of the logarithmic Sobolev constant for the canonical measure.

Our setting is as follows. Let $\Lambda \in \mathcal{F}_{l}$ be a finite set and, for a given boundary configuration $\tau$ and (possible vector) chemical potential $\underline{\lambda}$, let $\mu:=\mu_{\Lambda}^{\tau, \underline{\lambda}}$. Let $\left\{V_{\alpha}\right\}_{\alpha \in I}$ be a collection of subsets of $\Lambda$ such that $\operatorname{dist}\left(V_{\alpha}, V_{\beta}\right) \geqslant r+1$ for $\alpha \neq \beta, r$ being the range of the interaction, and let $V=\bigcup_{\alpha} V_{\alpha}$. Let also $f: \Omega_{\Lambda} \mapsto \mathbb{R}$ be such that $\mu(f)=0$ and define $\operatorname{Osc}_{\alpha}(f):=\left\|f-\mu_{V_{\alpha}}(f)\right\|_{\infty}$ and $\operatorname{Var}_{\alpha}(f):=\left\|\mu_{V_{\alpha}}^{\tau}(f, f)\right\|_{\infty}$.

PROPOSITION 5.1. - Fix $t_{0}>0$. Then, for all $t \in\left[0, t_{0}\right]$

$$
\mu\left(\mathrm{e}^{t f}\right) \leqslant \mathrm{e}^{t^{2} K_{f}},
$$


where $K_{f}=\mathrm{e}^{2\|\Phi\|} \sum_{x \in \Lambda}\left\|\nabla_{x} f\right\|_{\infty}^{2} c_{\mu}$ and $c_{\mu}$ is the logarithmic Sobolev constant of $\mu$ w.r.t. to the Heat Bath rates.

(ii) Let $f_{V}(\tau):=\mu_{V}^{\tau}(f)$. Then

$$
\mu\left(\mathrm{e}^{t f}\right) \leqslant \mathrm{e}^{t^{2} K_{f}^{\prime}}
$$

where

$$
K_{f}^{\prime}\left(t_{0}\right)=\mathrm{e}^{2\|\Phi\|} \sum_{x \in \Lambda \backslash V}\left\|\nabla_{x} f_{V}\right\|_{\infty}^{2} c_{\mu}+\sum_{\alpha} \operatorname{Var}_{\alpha}(f) \mathrm{e}^{t_{0} \operatorname{Osc}_{\alpha}(f)} .
$$

Remark. - Notice that if we make the trivial choice $V_{\alpha}=\Lambda$, then (ii) becomes $\mu\left(\mathrm{e}^{t f}\right) \leqslant \mathrm{e}^{t^{2} K_{f}^{\prime}}$ with $K_{f}^{\prime}=\mu(f, f) \mathrm{e}^{t_{0} \operatorname{Osc}(f)}$. Such a choice makes sense when $\operatorname{Osc}(f)$ is independent of $|\Lambda|$. However, in most of the subsequent application, $\operatorname{Osc}(f)=\mathrm{O}(|\Lambda|)$ and a more refined choice of the sets $V_{\alpha}$ will be necessary.

Proof. - In order to prove part (i) we appeal to the following lemma known as the Herbst's argument (see for instance Section 6 in [2]).

LEMMA 5.2. - Let $(\Omega, \mathcal{F}, \mu)$ be a finite probability space and $f$ a function on $\Omega$. Assume that there exists $K>0$ such that for all $t \in\left[0, t_{0}\right]$,

$$
\operatorname{Ent}_{\mu}\left(\mathrm{e}^{t f}\right) \leqslant K t^{2} \mu\left(\mathrm{e}^{t f}\right) .
$$

Then, for all $t \in\left[0, t_{0}\right]$,

$$
\mu\left(\mathrm{e}^{t f}\right) \leqslant \mathrm{e}^{t_{\mu}(f)+K t^{2}} .
$$

Proof. - Let $H(t)=\mu\left(\mathrm{e}^{t f}\right)$ be the Laplace transform of $f$. A simple computation gives

$$
t H^{\prime}(t)-H(t) \ln H(t)=\operatorname{Ent}\left(\mathrm{e}^{t f}\right) \leqslant K t^{2} H(t) .
$$

Dividing by $t^{2} H(t)$ and writing $K(t)=\frac{\ln H(t)}{t}$, we get

$$
K^{\prime}(t) \leqslant K(t)
$$

Notice now that $K(t) \rightarrow \mu(f)$ as $t \rightarrow 0$. Consequently, a simple integration achieves the proof.

Back to the proof of the proposition, if we apply the definition of the logarithmic Sobolev constant $c_{\mu}$ we get

$$
\operatorname{Ent}_{\mu}\left(\mathrm{e}^{t f}\right) \leqslant c_{\mu} \sum_{x \in \Lambda} \mu\left(c_{x}\left(\nabla_{x} \mathrm{e}^{\frac{t}{2} f}\right)^{2}\right) \leqslant\left(\mathrm{e}^{2\|\Phi\|} c_{\mu} \sum_{x \in \Lambda}\left\|\nabla_{x} f\right\|_{\infty}^{2}\right) t^{2} \mu\left(\mathrm{e}^{t f}\right),
$$

where $c_{x}(\sigma)=\mu_{\{x\}}^{\sigma}\left(\sigma^{x}\right)$ are the flip rates for the Heat Bath dynamics. Thus, thanks to Lemma 5.2, part (i) follows at once.

In order to prove part (ii) we proceed as follows. Denote by $\mu_{V}^{\tau}$ the Gibbs measure on $V$ with particle configuration $\tau$ in $\Lambda \backslash V$. By construction $\mu_{V}^{\tau}$ is a product measure over 
the sets $\left\{V_{\alpha}\right\}_{\alpha \in I}$ that, for simplicity and omitting the superscript $\tau$, we write as $\prod_{\alpha} \mu_{\alpha}$. Then we have

$$
\mu\left(\mathrm{e}^{t f}\right)=\mu\left(\left(\prod_{\alpha} \mu_{\alpha}\right)\left(\mathrm{e}^{t f}\right)\right) .
$$

Let us examine a generic term $\mu_{\alpha}\left(\mathrm{e}^{t f}\right)$. A simple Taylor expansion up to the second order gives

$$
\mu_{\alpha}\left(\mathrm{e}^{t f}\right) \leqslant \exp \left(t \mu_{\alpha}(f)+t^{2} \operatorname{Var}_{\alpha}(f) \mathrm{e}^{t_{0} \operatorname{Osc}_{\alpha}(f)}\right) .
$$

In order to iterate (over the index $\alpha$ ) the above bound, we simply observe that $\operatorname{Var}_{\beta}\left(\mu_{\alpha}(f)\right) \leqslant \operatorname{Var}_{\beta}(f)$ and $\operatorname{Osc}_{\beta}\left(\mu_{\alpha}(f)\right) \leqslant \operatorname{Osc}_{\beta}(f)$. Thus

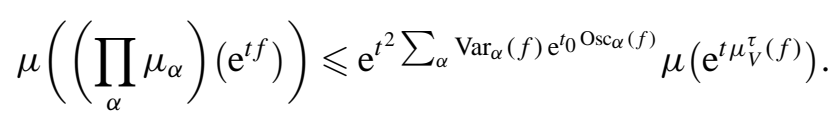

It suffices now to apply part (i) to the new term $\mu\left(\mathrm{e}^{t \mu_{V}^{\tau}(f)}\right)$.

Remark. - It is important to understand the difference between the two results given in Proposition 5.1. Assume that condition $\operatorname{UMST}(C, m, l)$ holds so that the logarithmic Sobolev constant $c_{\mu}$ is bounded from above uniformly in $\Lambda$ (see e.g. [16] and references therein) and let $f=\sum_{x \in \Lambda} f_{x}$, where $f_{x}$ is a mean zero local function with small support around $x$. For small values of the parameter $t$ one expects, on the basis of a second order Taylor expansion, a bound of the form

$$
\mu\left(\mathrm{e}^{t f}\right) \leqslant \mathrm{e}^{t^{2} K_{f}}
$$

with $K_{f} \approx \mu(f, f) \approx \sum_{x} \mu\left(f_{x}, f_{x}\right)$ because of the mixing assumption. Part (i) of the proposition proves the above Gaussian bound for all $t$, but with a new constant $K_{f} \approx c_{\mu} \sum_{x}\left\|\nabla_{x} f_{x}\right\|_{\infty}^{2}$. In "normal" situations that is quite satisfactory because $K_{f}$ becomes of the same order of the heuristic guess. The problem arises when, anomalous, very large values (maybe depending on the size of $\Lambda$ ) of the chemical potential $\underline{\lambda}$ are considered. In that case the Gibbs measure $\mu$ becomes very close to the product of highly unfair Bernoulli measures and its logarithmic Sobolev constant $c_{\mu}$ gets very large (see e.g. [12]) so that the Herbst's bound deteriorates. Part (ii) of the proposition partially takes care of these extreme situation, at least for not too large values of $t$, as we now explain (see also Proposition 5.3 below for more technical details). Assume that each set $V_{\alpha}$ is a cube of side $l_{0}$ multiple of the basic length scale $l$ and suppose that $f=\sum_{\alpha} f_{\alpha}$ where

$$
f_{\alpha}=\sum_{\substack{x \in V_{\alpha} \\ d\left(x, V_{\alpha}^{c}\right) \geqslant \frac{1}{2} l_{0}}} f_{x} .
$$

Then the constant $K_{f}^{\prime}$ appearing in part (ii) of the proposition becomes

$$
K_{f}^{\prime}=\mathrm{e}^{2\|\Phi\|} c_{\mu} \sum_{\alpha} \sum_{x \notin V_{\alpha}}\left\|\nabla_{x} \mu_{V_{\alpha}}\left(f_{\alpha}\right)\right\|_{\infty}^{2}+\sum_{\alpha} \operatorname{Var}_{\alpha}\left(f_{\alpha}\right) \mathrm{e}^{t_{0} \operatorname{Osc}_{\alpha}(f)}
$$


The second term in the above expression is, apart from the constant $\mathrm{e}^{t_{0} \mathrm{Osc}_{\alpha}(f)}$, exactly the contribution given by a naive Taylor expansion. The first term is instead similar to the constant $K_{f}$ discussed above but with the following important difference. The logarithmic Sobolev constant $c_{\mu}$ is now multiplied a term, $\sum_{x}\left\|\nabla_{x} \mu_{V_{\alpha}}\left(f_{\alpha}\right)\right\|_{\infty}^{2}$, which, apart from trivial constants, is different from zero only if $x \notin V_{\alpha}$ and in that case is proportional to $|V| \mathrm{e}^{-m l_{0}}$ because of the mixing assumptions. Moreover, for extreme values of the chemical potential, the "mass" $m$ can be taken proportional to the chemical potential itself so that the "effective" logarithmic Sobolev constant $\mathrm{e}^{-m l_{0}} c_{\mu}$ becomes very small and the Gaussian bound becomes more precise.

\subsection{An application at low density}

Here we discuss an application of our bound to a concrete case that will be important in the next section.

The setting is the standard multicanonical setting described at the beginning of Section 3.

Given now a local function $g$ with support $\Lambda_{g}$ containing the origin and of diameter smaller than $2 r, r$ being the range of interaction, and an integer $j \leqslant j_{\max }$, we define $G=\sum_{x \in \Lambda_{j}}\left[g_{x}-\mu\left(g_{x}\right)\right]$, where $g_{x}$ is the translate of $g$ by $x$. Let also $\rho_{j}:=\frac{N_{j}}{\left|\Lambda_{j}\right|}$ and assume without loss of generality that $\rho_{j} \leqslant 1 / 2$.

Proposition 5.3. - Assume $\operatorname{USMT}(C, m, l)$ and that there exists a constant $k=$ $k\left(\|\Phi\|_{\infty}, C, m, l\right)>0$ such that

$$
\begin{array}{ll}
\mu\left(\left|g_{x}\right|\right) \leqslant k \rho_{j}^{2}\|g\|_{\infty} & \forall x \in \Lambda_{j} \text { such that } \operatorname{dist}\left(x, \Lambda_{j}^{c}\right) \geqslant 2 r, \\
\mu\left(\left|g_{x}\right|\right) \leqslant k \rho_{j}\|g\|_{\infty} & \forall x \in \Lambda_{j} \text { such that } \operatorname{dist}\left(x, \Lambda_{j}^{c}\right) \leqslant 2 r .
\end{array}
$$

Then, for any $t_{0}>0$ there exist positive constants $A=A\left(\|\Phi\|_{\infty}, C, m, l, t_{0}, r,\|g\|_{\infty}, \delta_{0}\right)$ and $B=B\left(\|\Phi\|_{\infty}, C, m, l, r, \delta_{0}\right)$ such that

$$
\begin{aligned}
& \mu\left(\mathrm{e}^{t G}\right) \leqslant \mathrm{e}^{t^{2} A L^{d} \rho_{j}\left[\rho_{j}+\frac{\ln L}{L}\right]} \quad \forall t \in\left[0, t_{0}\right], \\
& v\left(\mathrm{e}^{t G}\right) \leqslant B \mathrm{e}^{t^{2} A L^{d} \rho_{j}\left[\rho_{j}+\frac{\ln L}{L}\right]} \quad \forall t \in\left[0, t_{0}\right] .
\end{aligned}
$$

Remark. - Although the proposition is stated for any value of the density $\rho_{j} \leqslant \frac{1}{2}$, the most interesting application is when $\rho_{j}$ is very small (possibly depending on $L$ ).

Proof. - In what follows $k$ will always denote a generic numerical constant depending only on $\|\Phi\|, C, m, l, t_{0}, r, d, \delta_{0},\|g\|_{\infty}$ and whose value may vary in different estimates.

Let $\left\{C_{\alpha}\right\}_{\alpha \in I}$ be a chessboard-like partition of $\Lambda_{j}$ into cubes of side $l_{0}=6 l, l$ being the basic length scale. A moment of thought suffices to convince oneself that we can partition the index set $I$ into $2 d$ subsets $\left\{I_{i}\right\}_{i=1}^{2 d}$ in such a way that $\min _{\left\{\alpha \neq \alpha^{\prime} \in I_{i}\right\}} \operatorname{dist}\left(C_{\alpha}, C_{\alpha^{\prime}}\right) \geqslant l_{0}$ and $\min _{\alpha \in I_{i}} \operatorname{dist}\left(C_{\alpha}, \Lambda_{j}^{c}\right) \geqslant l_{0}$. Accordingly we write $G=\sum_{i=1}^{2 d} G^{(i)}+G^{\text {ext }}$, where

$$
G^{\mathrm{ext}}=\sum_{\substack{x \in \Lambda_{j} \\ \operatorname{dist}\left(x, \Lambda_{j}^{c}\right) \leqslant l_{0}}}\left[g_{x}-\mu\left(g_{x}\right)\right] \quad \text { and } \quad G^{(i)}=\sum_{\alpha \in I_{i}} g_{\alpha} \quad \text { with } g_{\alpha}=\sum_{x \in C_{\alpha}}\left[g_{x}-\mu\left(g_{x}\right)\right] \text {. }
$$


Thus, using Hölder inequality, we arrive at

$$
\mu\left(\mathrm{e}^{t G}\right) \leqslant \mu\left(\mathrm{e}^{(2 d+1) t G^{\mathrm{ext}}}\right)^{\frac{1}{2 d+1}} \prod_{i=1}^{2 d} \mu\left(\mathrm{e}^{(2 d+1) t G^{(i)}}\right)^{\frac{1}{2 d+1}} .
$$

It is thus enough to bound from above a generic term $\mu\left(\mathrm{e}^{(2 d+1) t G^{(i)}}\right)$ and the boundary term $\mu\left(\mathrm{e}^{t G^{\text {ext }}}\right)$. Let us thus set $f:=G^{(i)}$ and, for any $\alpha \in I_{i}$, let $V_{\alpha}$ be a cube of side $2 l_{0}$ and having the same center of $C_{\alpha}$. Thanks to our assumptions on the function $g$ and the strong mixing, the constants appearing in the second part of Proposition 5.1 satisfy the bounds

$$
\begin{array}{r}
\operatorname{Var}_{\alpha}(f) \leqslant k l_{0}^{d} \rho_{j}^{2}, \\
\operatorname{Osc}_{\alpha}(f) \leqslant k l_{0}^{d}, \\
\sup _{x \notin V_{\alpha}}\left\|\nabla_{x} \mu_{\alpha}(f)\right\|_{\infty}^{2} \leqslant k \rho_{j}^{3 l},
\end{array}
$$

where the latter inequality follows from any standard low activity expansion if $\rho_{j}$ is small and from the mixing assumption if $\rho_{j}$ is "moderate".

Let now $\mu_{j}:=\mu_{\Lambda_{j}}^{\tau}$ and write $\mu\left(\mathrm{e}^{t f}\right)=\mu\left(\mu_{j}\left(\mathrm{e}^{t f}\right)\right)$. It is not difficult to check that the logarithmic Sobolev constant of the measure $\mu_{j}, c_{\mu_{j}}$, is not larger than $k \ln \left(\frac{1}{\rho_{j}}\right)$ uniformly in the boundary condition $\tau$, because of the mixing assumption and the fact that $-\ln (p)$ is the order of magnitude of the logarithmic Sobolev constant of a Bernoulli measure of parameter $p$ as $p \rightarrow 0$ (see e.g. [12]). If we now apply the bound (ii) of Proposition 5.1 to $\mu_{j}\left(\mathrm{e}^{t f}\right)$ we get

$$
\mu\left(\mu_{j}\left(\mathrm{e}^{t f}\right)\right) \leqslant \mu\left(\mathrm{e}^{t \mu_{j}(f)}\right) \mathrm{e}^{t^{2} k \rho_{j}^{2}\left|\Lambda_{j}\right|} \quad \forall t \in\left[0, t_{0}\right] .
$$

It remains to bound the correction term $\mu\left(\mathrm{e}^{t \mu_{j}(f)}\right)$. It suffices to apply part (i) of Proposition 5.1 together with the observation that $c_{\mu}<k \ln L$ because of the previous remarks and that

$$
\sum_{x \in \Lambda_{j}^{c}}\left\|\nabla_{x} \mu_{j}(f)\right\|_{\infty}^{2} \leqslant k L^{d-1} \rho_{j}^{6 l}
$$

because of the mixing assumption and the hypothesis $\min _{\alpha \in I_{i}} \operatorname{dist}\left(C_{\alpha}, \Lambda_{j}^{c}\right) \geqslant l_{0}$ (see also (5.4)). In conclusion

$$
\mu\left(\mathrm{e}^{t \mu_{j}(f)}\right) \leqslant \mathrm{e}^{t^{2} k \rho_{j}^{6 l} L^{d-1} \ln L} \leqslant \mathrm{e}^{t^{2} k \rho_{j}^{2}\left|\Lambda_{j}\right|},
$$

so that

$$
\mu\left(\mathrm{e}^{(2 d+1) t G^{(i)}}\right) \leqslant \mathrm{e}^{t^{2} k \rho_{j}^{2}\left|\Lambda_{j}\right|} .
$$

Let us finally analyze the boundary term $\mu\left(\mathrm{e}^{t G^{\text {ext }}}\right)$. We could repeat word by word the argument that was used to bound the term $\mu\left(\mathrm{e}^{t \mu_{j}(f)}\right)$ to get

$$
\mu\left(\mathrm{e}^{t G^{\mathrm{ext}}}\right) \leqslant \mathrm{e}^{t^{2} k L^{d-1} \ln L}
$$


which is certainly smaller than the r.h.s. of (5.5) for $\rho_{j}^{2} \geqslant \frac{\ln (L)}{L}$. If instead $\rho_{j}^{2} \leqslant \frac{\ln (L)}{L}$ then we proceed as follows. We decompose $G^{\text {ext }}$ into the sum of a finite (only depending on $r$ ) number of terms $G^{\text {ext, } i}$, each of the form $G^{\text {ext, } i}=\sum_{x \in E_{i}}\left[g_{x}-\mu\left(g_{x}\right)\right]$ where $E_{i}$ is a subset of the "layer" $\left\{x \in \Lambda_{j}\right.$ such that $\left.\operatorname{dist}\left(x, \Lambda_{j}^{c}\right) \leqslant l_{0}\right\}$ and it has the property that $\min _{x, y \in E_{i}} \operatorname{dist}(x, y) \geqslant 2 r$. The choice of the sets $E_{i}$ is somewhat arbitrary but it causes no problem (just write $\mathbb{Z}^{d}=\bigcup_{x \in 2 r \mathbb{Z}^{d}} Q_{2 r}(x)$ ).

Given $x \in E_{i}$, we let $V_{x}=\Lambda_{g}+x$ and we bound from above the quantity $\mu\left(\mathrm{e}^{t G^{\mathrm{ext}, i}}\right)$ using part (ii) of Proposition 5.1 with the sets $\left\{V_{x}\right\}_{x \in E_{i}}$ playing the role of the sets $\left\{V_{\alpha}\right\}_{\alpha \in I}$. With the obvious notation and thanks to our hypotheses on the function $g$ we get

$$
\begin{aligned}
\operatorname{Var}_{x}\left(g_{x}\right) & \leqslant k\|g\|_{\infty}^{2} \rho_{j}, \\
\operatorname{Osc}_{x}\left(g_{x}\right) & \leqslant 2\|g\|_{\infty}, \\
\sup _{y \notin V_{x}}\left\|\nabla_{y} \mu_{V_{x}}\left(g_{x}\right)\right\|_{\infty}^{2} & \leqslant k\|g\|_{\infty}^{2} \rho_{j} .
\end{aligned}
$$

Thus

$$
\mu\left(\mathrm{e}^{t G^{\mathrm{ext}, i}}\right) \leqslant \mathrm{e}^{t^{2} c_{\mu} \rho_{j} L^{d-1}} \leqslant \mathrm{e}^{t^{2} k \rho_{j} L^{d-1} \ln L} \quad \forall t \in\left[0, t_{0}\right] .
$$

In the last inequality we have used the bound $c_{\mu} \leqslant k \ln L$. In conclusion, by putting together (5.5) and (5.6) we get

$$
\mu\left(\mathrm{e}^{t G}\right) \leqslant \mathrm{e}^{t^{2} A L^{d} \rho_{j}\left[\rho_{j}+\frac{\ln L}{L}\right]} \quad \forall t \in\left[0, t_{0}\right]
$$

for a suitable positive constant $A=A\left(\|\Phi\|_{\infty}, C, m, l, t_{0}, r, d,\|g\|_{\infty}, \delta_{0}\right)$.

In order to prove the analogous bound for the multicanonical measure $v$, we observe that the function $G$ can be written as $G=G_{1}+G_{2}$, where $G_{1}$ and $G_{2}$ have the same expression of $G$ but with the sum over $x$ restricted to two halves of the set $\Lambda_{j}$. Then

$$
v\left(\mathrm{e}^{t G}\right) \leqslant v\left(\mathrm{e}^{2 t G_{1}}\right)^{\frac{1}{2}} v\left(\mathrm{e}^{2 t G_{2}}\right)^{\frac{1}{2}}
$$

and we can apply to each of the factors the bound (see Proposition 3.3)

$$
v\left(\mathrm{e}^{2 t G_{i}}\right) \leqslant B \mu\left(\mathrm{e}^{2 t G_{i}}\right), \quad i=1,2 .
$$

The final result follows at once from the bound on the grand canonical expectation.

\subsection{An application to "normal" densities}

Here we discuss another situation, similar to the one just analyzed, that will play an important role in the forthcoming sections. Contrary to Proposition 5.3, the interest of the results stated below is when the density $\rho_{j}$ is not too small. That should explains why, in most of the estimates given below, we allow certain numerical constant to depend in some unspecified way on $\rho_{j}$, without worrying about the case when $\rho_{j} \rightarrow 0$ as $L \rightarrow \infty$.

The setting is that already described in the previous paragraph but the function $G$ is different. In order to define it, let $\left\{C_{\alpha}\right\}_{\alpha \in I}$ be a collection of cubes in $\Lambda_{j}$ of sides 
$l_{0} \gg r$ multiple of $l$, such that for any $\alpha \neq \beta, d\left(C_{\alpha}, C_{\beta}\right) \geqslant l_{0}^{1 / 2}$ and $d\left(C_{\alpha}, \partial \Lambda\right) \geqslant l_{0}^{1 / 2}$. Let $C_{\alpha}^{\text {int }}:=\left\{x \in C_{\alpha}: d\left(x, C_{\alpha}^{c}\right) \geqslant l_{0}^{1 / 4}\right\}$, let $C^{\text {int }}=\bigcup_{\alpha} C_{\alpha}^{\text {int }}$ and denote by $n_{c}=|I|$ the number of such cubes.

Given $\eta \in \Omega_{\Lambda}$ and $s \in\left[0, l_{0}^{d}\right]$, write $\mu_{\alpha}^{\eta, s}(\cdot)=\mu_{C_{\alpha}}^{\eta, \lambda(\eta, s)}(\cdot)$ to denote the grand canonical Gibbs measure on $C_{\alpha}$ with boundary condition $\eta$ and constant chemical potential $\lambda(\eta, s)$ such that $\mu_{C_{\alpha}}^{\eta, \lambda(\eta, s)}\left(N_{C_{\alpha}}\right)=s$. Whenever $s$ is also an integer, say $s=n \in\left[0,1, \ldots,\left\lfloor l_{0}^{d}\right\rfloor\right]$, we will use the standard notation $v_{C_{\alpha}, n}^{\eta}$ for the corresponding canonical Gibbs measure.

Consider now a local function $g$ with support $\Lambda_{g}$ containing the origin and of diameter smaller than $2 r$ such that $g(\underline{0})=0$ where $\underline{0}$ is the configuration identically equal to zero. Then we define $g^{\delta}(\sigma)=g_{x}(\sigma)-\delta \sigma(x)$ and

$$
\begin{array}{ll}
\xi_{\alpha}^{\delta}(\eta, s)=\sum_{x \in C_{\alpha}^{\mathrm{int}}} \mu_{\alpha}^{\eta, s}\left(g_{x}^{\delta}\right), \quad \xi_{\alpha}^{\delta}(\eta):=\xi_{\alpha}^{\delta}\left(\eta, N_{C_{\alpha}}(\eta)\right), \quad \xi_{\alpha}^{\delta, \underline{0}}(\eta):=\xi_{\alpha}^{\delta}\left(\underline{0}, N_{C_{\alpha}}(\eta)\right) \\
g_{\alpha}^{\delta}(\eta, n)=\sum_{x \in C_{\alpha}^{\mathrm{int}}} v_{C_{\alpha}, n}^{\eta}\left(g_{x}^{\delta}\right) ; \quad g_{\alpha}^{\delta}(\eta):=g_{\alpha}^{\delta}\left(\eta, N_{C_{\alpha}}(\eta)\right)
\end{array}
$$

where $\delta$ is such that for one (and therefore all) cube $C_{\alpha}:\left.\frac{\mathrm{d}}{\mathrm{d} s} \xi_{\alpha}^{\delta, \underline{0}}(s)\right|_{s=\bar{n}}=0, \bar{n}=\rho_{j} l_{0}^{d}$. By definition

$$
\delta=\frac{\mu_{C_{\alpha}}^{\underline{0}, \lambda(\underline{0}, \bar{n})}\left(\sum_{x \in C_{\alpha}^{\text {int }}} g_{x}, N_{C_{\alpha}}\right)}{\mu_{C_{\alpha}}^{\underline{0} \lambda(\underline{0}, \bar{n})}\left(N_{C_{\alpha}^{\text {int }}}, N_{C_{\alpha}}\right)}
$$

so that, if condition $\operatorname{USMT}(C, m, l)$ holds, $\delta \leqslant \delta\left(C, m, l,\|g\|_{\infty}\right)$ uniformly in $L$.

Finally we define

$$
\begin{aligned}
G_{v}(\eta) & :=\sum_{\alpha}\left[g_{\alpha}^{\delta}(\eta)-\mu\left(g_{\alpha}^{\delta}\right)\right], \\
G_{\mu}(\eta) & :=\sum_{\alpha}^{\alpha}\left[\xi_{\alpha}^{\delta}(\eta)-\mu\left(\xi_{\alpha}^{\delta}\right)\right], \\
G_{\mu}^{0}(\eta) & :=\sum_{\alpha}\left[\xi_{\alpha}^{\delta, 0}(\eta)-\mu\left(\xi_{\alpha}^{\delta, \underline{0}}\right)\right], \\
G^{\operatorname{ext}}(\eta) & :=\sum_{x \in \Lambda_{j} \backslash C^{\text {int }}}\left[g_{x}^{\delta}-\mu\left(g_{x}^{\delta}\right)\right] .
\end{aligned}
$$

Proposition 5.4. - Assume USMT $(C, m, l)$ and fix $t_{0}>0$. Then there exists $a$ constant $A=A\left(C, m, l, t_{0},\|g\|_{\infty}, \rho_{j}\right)$ such that

$$
\begin{aligned}
& \mu\left(\mathrm{e}^{t G^{\mathrm{ext}}}\right) \leqslant \mathrm{e}^{t^{2} L^{d} A l_{0}^{-1 / 2}} \quad \forall t, \\
& \mu\left(\mathrm{e}^{t\left(G_{\nu}-G_{\mu}\right)}\right) \leqslant \mathrm{e}^{t^{2} L^{d} A l_{0}^{-d}} \quad \forall t \in\left[0, t_{0}\right], \\
& \mu\left(\mathrm{e}^{t\left(G_{\mu}^{\bar{\mu}}-G_{\mu}\right)}\right) \leqslant \mathrm{e}^{t^{2} L^{d} A l_{0}^{-1}} \quad \forall t, \\
& \mu\left(\mathrm{e}^{t G^{\frac{0}{\mu}}}\right) \leqslant \mathrm{e}^{t^{2} L^{d} A l_{0}^{-d}} \quad \forall t \in\left[0, t_{0} l_{0}^{-d}\right] .
\end{aligned}
$$

The same bounds hold, but with an extra factor $B=B\left(\|\Phi\|_{\infty}, C, m, l, r, \delta\right)$ in front of the exponential, if $\mu$ is replaced by the multicanonical measure $\nu$. 
Proof. - In what follows $k$ will always denote a generic numerical constant depending only on $\|\Phi\|, C, m, l, t_{0}, r, d,\|g\|_{\infty}$ and whose value may vary in different estimates.

(i). Let $\mu_{j}:=\mu_{\Lambda_{j}}^{\tau}$ and write $\mu\left(\mathrm{e}^{t G^{\mathrm{ext}}}\right)=\mu\left(\mu_{j}\left(\mathrm{e}^{t G^{\mathrm{ext}}}\right)\right)$. We simply apply part (i) of Proposition 5.1 to the function $\mu_{j}\left(\mathrm{e}^{t G^{\text {ext }}}\right)$ together with the observation that, thanks to the mixing condition, $\sup _{\tau} c_{\mu_{j}^{\tau}} \leqslant k\left(C, m, l, \rho_{j}, \delta\right)$. The reason why we refrain to apply directly such an argument to $\mu\left(\mathrm{e}^{t G^{\text {ext }}}\right)$ is that it is possible that the global logarithmic Sobolev constant $c_{\mu}$ is very large, up to order $k \ln L$, because in some atom $\Lambda_{i}, i \neq j$, the density is either very small or very close to one. As we will see, conditioning on the exterior of $\Lambda_{j}$ is a simple way to take care of such a nasty possibility. Since

$$
\sum_{x \in \Lambda_{j}}\left\|\nabla_{x} G^{\mathrm{ext}}\right\|_{\infty}^{2} \leqslant k L^{d} l_{0}^{-\frac{1}{2}}
$$

we get

$$
\mu\left(\mu_{j}\left(\mathrm{e}^{t G^{\mathrm{ext}}}\right)\right) \leqslant \mu\left(\mathrm{e}^{t \mu_{j}\left(G^{\mathrm{ext}}\right)}\right) \mathrm{e}^{t^{2} k L^{d} l_{0}^{-\frac{1}{2}}} .
$$

The term $\mu\left(\mathrm{e}^{t \mu_{j}\left(G^{\mathrm{ext}}\right)}\right)$ can also be estimated by the Herbst argument if we recall the observation that, under the mixing assumption, $c_{\mu} \leqslant k \ln L$ (see the proof of Proposition 5.3). We get

$$
c_{\mu} \sum_{x \in \Lambda}\left\|\nabla_{x} \mu_{j}^{\tau}\left(G^{\mathrm{ext}}\right)\right\|_{\infty}^{2} \leqslant k L^{d-1} \ln L
$$

so that

$$
\mu\left(\mathrm{e}^{t \mu_{j}\left(G^{\mathrm{ext}}\right)}\right) \leqslant \mathrm{e}^{t^{2} k L^{d-1} \ln L} .
$$

Clearly (5.9) and (5.10) complete the proof.

(ii). First we observe that, because of the equivalence of ensembles (see Proposition 3.2 point (1)), $\sup _{\alpha}\left\|\left(g_{\alpha}^{\delta}-\xi_{\alpha}^{\delta}\right)\right\|_{\infty} \leqslant k$. Next, given $\alpha$, let $V_{\alpha}$ be an $\left(\frac{1}{2} l_{0}^{1 / 2}-2 r\right)$ neighborhood of the cube $C_{\alpha}$. For $l_{0}$ large enough the sets $\left\{V_{\alpha}\right\}$ satisfy the condition of Proposition 5.1. Moreover

$$
\left\|\nabla_{x} \mu_{V_{\alpha}}\left(g_{\alpha}^{\delta}-\xi_{\alpha}^{\delta}\right)\right\|_{\infty} \leqslant \begin{cases}k \mathrm{e}^{-\frac{1}{2} m l_{0}^{\frac{1}{2}}} & \text { if } x \in \partial_{r}^{+} V_{\alpha}, \\ 0 & \text { otherwise }\end{cases}
$$

for $l_{0}$ large enough.

Thus, part (ii) of Proposition 5.1 together the usual conditioning $\mu(\cdot)=\mu\left(\mu_{j}(\cdot)\right)$ give (see also the argument leading to formula (5.10))

$$
\begin{aligned}
\mu\left(\mathrm{e}^{t\left(G_{\nu}-G_{\mu}\right)}\right) & \leqslant \mathrm{e}^{t^{2} L^{d} k l_{0}^{-d}} \mu\left(\mathrm{e}^{t \mu_{j}\left(G_{\nu}-G_{\mu}\right)}\right), \\
& \leqslant \mathrm{e}^{t^{2} L^{d} k\left(l_{0}^{-d}+L^{-1} \ln L\right)} \\
& \leqslant \mathrm{e}^{t^{2} L^{d} A l_{0}^{-d}} \quad \forall t \in\left[0, t_{0}\right]
\end{aligned}
$$

for $L$ large enough. 
(iii). Notice that

$$
\left\|\nabla_{x}\left(\xi_{\alpha}^{\delta}(\eta)-\xi_{\alpha}^{\delta, \underline{0}}(\eta)\right)\right\|_{\infty} \leqslant \begin{cases}k & \text { if } x \in \partial_{r}^{+} C_{\alpha} \\ k l_{0}^{-1} & \text { if } x \in C_{\alpha}\end{cases}
$$

because of Lemma 3.1 point (i) of (1) and (2). Therefore we can apply the same reasoning leading to $(5.11)$ to get

$$
\mu\left(\mathrm{e}^{t\left(G \frac{0}{\mu}-G_{\mu}\right)}\right) \leqslant \mathrm{e}^{t^{2} L^{d} A\left(l_{0}^{-1}+L^{-1} \ln L\right)} \quad \forall t
$$

and the sought bound follows for $L$ large enough.

(iv). We want to use part (ii) of Proposition 5.1.

As in the proof of point (ii), let $V_{\alpha}$ be an $\left(\frac{1}{2} l_{0}^{\frac{1}{2}}-2 r\right)$-neighborhood of the cube $C_{\alpha}$. For $l_{0}$ large enough the sets $\left\{V_{\alpha}\right\}$ satisfy the condition of Proposition 5.1 and because of the mixing assumption

$$
\left\|\nabla_{x} \mu_{V_{\alpha}}\left(\xi_{\alpha}^{\delta, \underline{0}}\right)\right\|_{\infty} \leqslant \begin{cases}k \mathrm{e}^{-\frac{1}{2} m l_{0}^{\frac{1}{2}}} & \text { if } x \in \partial_{r}^{+} V_{\alpha} \\ 0 & \text { otherwise }\end{cases}
$$

for $l_{0}$ large enough.

Let us now compute $\operatorname{Var}_{\alpha}\left(\xi_{\alpha}^{\delta,} \underline{0}\right)$ and $\operatorname{Osc}_{\alpha}\left(\xi_{\alpha}^{\delta,} \underline{0}\right)$. For this purpose we first observe, that, by the very definition of $\delta$ and the constance of the chemical potential inside $\Lambda_{j}$, we have (see point (ii) of part (2) of Lemma 3.1)

$$
\xi_{\alpha}^{\delta}(\underline{0}, n)-\xi_{\alpha}^{\delta}(\underline{0}, \bar{n})=\int_{\bar{n}}^{n} \mathrm{~d} s \int_{\bar{n}}^{s} \mathrm{~d} t \frac{\mathrm{d}^{2}}{\mathrm{~d} t^{2}} \xi_{\alpha}^{\delta}(\underline{0}, t) \leqslant k \frac{(n-\bar{n})^{2}}{\bar{n}} .
$$

Therefore $\operatorname{Osc}_{\alpha}\left(\xi_{\alpha}^{\delta,} \underline{0}\right) \leqslant k l_{0}^{d}$ and $\left(n_{\alpha}:=N_{C_{\alpha}}(\eta)\right)$

$$
\begin{aligned}
\operatorname{Var}_{\alpha}\left(\xi_{\alpha}^{\delta, \underline{0}}\right) & \leqslant \frac{k}{l_{0}^{2 d}}\left\|\mu_{V_{\alpha}}\left(\left(n_{\alpha}-\bar{n}\right)^{4}\right)\right\|_{\infty} \\
& \leqslant \frac{k}{l_{0}^{2 d}}\left\|\mu_{V_{\alpha}}\left(\left(n_{\alpha}-\mu_{V_{\alpha}}\left(n_{\alpha}\right)\right)^{4}\right)\right\|_{\infty}+\frac{k}{l_{0}^{2 d}}\left\|\mu_{V_{\alpha}}\left(n_{\alpha}\right)-\bar{n}\right\|_{\infty}^{4} \\
& \leqslant k
\end{aligned}
$$

again because of the mixing condition. The statement now follows at once from point (ii) of Proposition 5.1 applied to $f=G^{\frac{0}{\mu}}$.

Finally the statements for the multicanonical measure follow exactly as in the proof of Proposition 5.3.

\section{On the covariance of $\boldsymbol{f}^{2}$ with sums of local functions}

In this section we discuss some important bounds on covariances of the form $v\left(f^{2}, G\right)$, where $v$ is a multicanonical measure on $\Lambda=\bigcup_{j=1}^{j_{\max }} \Lambda_{j}$ as in the standard 
multicanonical setting of Section $3, f$ an arbitrary function with $v\left(f^{2}\right)=1$ and $G=$ $\sum_{x \in \Lambda_{j}} g_{x}$ for some $1 \leqslant j \leqslant j_{\max }$, where $g_{x}$ is the translate by $x$ of a local function $g$ with small support around the origin.

Roughly speaking our main goal is to bound $v\left(f^{2}, G\right)^{2}$ in terms of the only quantities that enter in the logarithmic Sobolev inequality, namely the entropy $\operatorname{Ent}_{v}\left(f^{2}\right)$ and the Dirichelt form $\mathcal{E}_{v}(f, f)$, but we want to keep track of the right dependence on the volume or, more precisely, on the number of particles $N_{j}$ in $\Lambda_{j}$. Moreover, for reasons that will become more clear later in the paper, we have another constraint in that $\operatorname{Ent}_{v}\left(f^{2}\right)$ must appear multiplied by a very small constant times the volume.

In order to appreciate the difficulty of the problem, we notice that, since $f$ enters as $f^{2}$, one of the natural tool to bound covariances, namely Schwarz inequality, becomes useless since no $L^{p}$-norm of $f, p>2$, enters into the logarithmic Sobolev inequality. This is precisely one of the main technical difference and new challenge between the Poincaré inequality (where $f$ appears linearly) and the logarithmic Sobolev inequality for conservative stochastic dynamics.

A natural counterpart to Schwarz inequality in this context is the so called entropy inequality that can be stated as follows.

LEMMA 6.1. - Let $(\Omega, \mathcal{F}, \mu)$ be a finite probability space. Then, for any $t>0$ and any real valued functions $f, G$ on $\Omega$ with $\mu\left(f^{2}\right)=1$,

$$
\mu\left(f^{2} G\right) \leqslant \frac{1}{t} \ln \left(\mu\left(\mathrm{e}^{t G}\right)\right)+\frac{1}{t} \operatorname{Ent}_{\mu}\left(f^{2}\right) .
$$

Proof. - It is an immediate consequence of the following Young's inequality, valid for all $u>0$ and all $v: u v \leqslant u \ln u+\mathrm{e}^{v}-u$.

In our case we can assume $G$ of zero mean w.r.t. $\mu$ (because we are taking the covariance of $G$ with $f^{2}$ ) so that it is natural to expect (see Section 5) a Gaussian bound of the form $v\left(\mathrm{e}^{t G}\right) \leqslant \mathrm{e}^{t^{2} K\left|\Lambda_{j}\right|}$ for all $t>0$ and some constant $K$ that may depend on the particle density. If that is the case, Lemma 6.1 gives

$$
v\left(f^{2} G\right) \leqslant t K\left|\Lambda_{j}\right|+\frac{1}{t} \operatorname{Ent}_{v}\left(f^{2}\right) \quad \forall t>0 .
$$

If we finally optimize over the free parameter $t$, namely we take $t^{2}=\frac{1}{K\left|\Lambda_{j}\right|} \operatorname{Ent}_{v}\left(f^{2}\right)$, and we assume, without loss of generality, that $v\left(f^{2} G\right) \geqslant 0$, we get

$$
v\left(f^{2} G\right)^{2} \leqslant 4 K\left|\Lambda_{j}\right| \operatorname{Ent}_{v}\left(f^{2}\right) .
$$

Let us pause for a moment to clarify (for the alert reader only) more explicitely how we plan to use the results of Section 5. In that section in fact, Gaussian bounds on the canonical Laplace transform of $G$ were established under the hypothesis of vanishing grand canonical (not of the canonical) average of $G$. Moreover the resulting estimates are distorted Gaussian bounds because of the presence of an extra constant $B$ in front of 
the exponential. Thus, in reality, (6.2) is slightly more complicated and looks like

$$
v\left(f^{2}, G\right) \leqslant \mu(G)+\frac{1}{t} \ln B+t K\left|\Lambda_{j}\right|+\frac{1}{t} \operatorname{Ent}_{\mu}\left(f^{2}\right) \quad \forall t>0 .
$$

The two extra terms, $\mu(G)$ and $\frac{1}{t} \ln B$ are just a little nuisance; the first one can in fact be handled quite easily using the equivalence of ensembles while the second one becomes harmless by choosing the "optimal" $t$ always greater than $\left|\Lambda_{j}\right|^{-\frac{1}{2}}$.

Let us continue our informal discussion. Let $t_{*}^{2}=\frac{1}{\left|\Lambda_{j}\right|} \operatorname{Ent}_{v}\left(f^{2}\right)$ and let $t_{0}$ be some large number independent of the volume and particle density. If $t_{*} \geqslant t_{0}$, i.e. $\left|\Lambda_{j}\right| \leqslant$ $\frac{1}{t_{0}^{2}} \operatorname{Ent}_{v}\left(f^{2}\right)$, then trivially

$$
v\left(f^{2} G\right)^{2} \leqslant\|G\|_{\infty}^{2} \leqslant\|g\|_{\infty}^{2}\left|\Lambda_{j}\right| \leqslant\|g\|_{\infty}^{2} \frac{1}{t_{0}^{2}}\left|\Lambda_{j}\right| \operatorname{Ent}_{v}\left(f^{2}\right)
$$

which is like (6.3) but with a smaller constant in front of $\left|\Lambda_{j}\right| \operatorname{Ent}_{v}\left(f^{2}\right)$ if $t_{0}$ is large enough. In other words, if $t_{*} \geqslant t_{0}$ a simple $L_{\infty}$ estimate gives a better result than the entropy bound.

In order to understand this point we remark that, on the basis of the central limit theorem and for "normal" values of the particle density in $\Lambda_{j}$, one expects the distribution $d P(G)$ of the random variable $\sum_{x \in \Lambda_{j}} g_{x}$ to be close to a centered gaussian with variance proportional to $\left|\Lambda_{j}\right|$. If this is the case, then, for $t$ large enough, the distorted distribution $\mathrm{d} P_{t}(G) \propto \mathrm{e}^{t G} \mathrm{~d} P(G)$ becomes concentrated on the largest value of $G$ and the gaussian bound $v\left(\mathrm{e}^{t G}\right) \leqslant \mathrm{e}^{t^{2} K\left|\Lambda_{j}\right|}$ becomes unnatural and certainly worst than the trivial one $v\left(\mathrm{e}^{t G}\right) \leqslant \mathrm{e}^{t\|G\|_{\infty}}$. On the contrary, for "moderate" values of $t$, the distortion only moves the center of the gaussian and in this case the entropy inequality will perform better.

Thus, in what follows, our strategy will always be, roughly speaking, the following. Depending on the ratio between the entropy and the volume, we will either apply the entropy bound with the optimal $t$ and appeal several times to the results of Section 5 or we will apply the trivial $L_{\infty}$ bound. It remains to explain how we get in both cases a small constant in front of the entropy. For small values of the density or large values of the entropy it will follow quite easily from the results of Section 5 (see Proposition 5.3). In all the other cases we will have to appeal to a partial average argument, almost identical to the one used in [14] and [10] under the name of "two-blocks estimates", in order to reduce the fluctuations of the function $G$.

We now explain more precisely our results.

\subsection{Low density case}

Here we discuss our first result in the low density regime.

Proposition 6.2. - Assume $\operatorname{USMT}(C, m, l)$ and that there exists a constant $k=$ $k\left(\|\Phi\|_{\infty}, C, m, l\right)>0$ such that

$$
\mu\left(\left|g_{x}\right|\right) \leqslant k \rho_{j}^{2}\|g\|_{\infty} \quad \forall x \in \Lambda_{j} \text { such that } \operatorname{dist}\left(x, \Lambda_{j}^{c}\right) \geqslant 2 r,
$$




$$
\begin{aligned}
\mu\left(\left|g_{x}\right|\right) & \leqslant k \rho_{j}\|g\|_{\infty} \quad \forall x \in \Lambda_{j} \text { such that } \operatorname{dist}\left(x, \Lambda_{j}^{c}\right) \leqslant 2 r, \\
\|G\|_{\infty} & \leqslant k N_{j} .
\end{aligned}
$$

Then, for any $\varepsilon>0$, there exists $C_{\varepsilon}$ and $\rho_{0}$ such that for all $\rho_{j}<\rho_{0}$, for all function $f$ with $v\left(f^{2}\right)=1$,

$$
v\left(f^{2}, G\right)^{2} \leqslant N_{j}\left(C_{\varepsilon}+\varepsilon \operatorname{Ent}_{v}\left(f^{2}\right)\right)
$$

for L large enough.

Proof. - We can assume $\mu(G)=0$. As usual, in what follows, $k$ will always denote a generic positive numerical constant depending only on $\|\Phi\|, C, m, l, r, d, \delta_{0},\|g\|_{\infty}$ and whose value may vary in different estimates.

Fix $\varepsilon>0$ and $f$ with $v\left(f^{2}\right)=1$. Let $M>0$ be an arbitrary, large constant that will be fixed further on and let $t_{*}^{2}=\frac{1}{N_{j}}\left(1 \vee M \operatorname{Ent}_{v}\left(f^{2}\right)\right)$. We distinguish between two cases.

In the first one, we suppose $t_{*} \geqslant M$. According to the general discussion we can safely apply an $L_{\infty}$ bound to get

$$
v\left(f^{2}, G\right)^{2} \leqslant 2\|G\|_{\infty}^{2} \leqslant k N_{j}^{2} \leqslant \frac{k}{M} N_{j} \operatorname{Ent}_{v}\left(f^{2}\right),
$$

where we have used the hypotheses on $\|G\|_{\infty}$ and the definition of $t_{*}$.

In the second case, we suppose $t_{*} \leqslant M$ and we first write

$$
v\left(f^{2}, G\right)^{2} \leqslant 2 v\left(f^{2} G\right)^{2}+2 v(G)^{2} .
$$

Using the equivalence of ensembles (see Proposition 3.3) and the fact that $\mu(G)=0$, $|v(G)| \leqslant k$ for some constant $k$. Thus, we can focus our attention on the first term $v\left(f^{2} G\right)^{2}$.

By applying Proposition 5.3 with $t_{0}=M$, we get that there exist constants $A, B$ depending on $\|\Phi\|_{\infty}, C, m, l, t_{0}, r,\|g\|_{\infty}, \delta_{0}$ such that

$$
v\left(\mathrm{e}^{t_{*} G}\right) \leqslant B \mathrm{e}^{t_{*}^{2} A L^{d} \rho_{j}\left[\rho_{j}+\frac{\ln L}{L}\right]}=B \mathrm{e}^{t_{*}^{2} A N_{j}\left[\rho_{j}+\frac{\ln L}{L}\right]} .
$$

Thus, by applying Lemma 6.1 with $t=t_{*}$, we get

$$
\begin{aligned}
v\left(f^{2} G\right)^{2} & \leqslant\left(\frac{1}{t_{*}} \ln B+t_{*} A N_{j}\left[\rho_{j}+\frac{\ln L}{L}\right]+\frac{1}{t_{*}} \operatorname{Ent}_{v}\left(f^{2}\right)\right)^{2} \\
& \leqslant k N_{j}\left(1+\left(M A^{2}\left[\rho_{j}+\frac{\ln L}{L}\right]^{2}+\frac{1}{M}\right) \operatorname{Ent}_{v}\left(f^{2}\right)\right) .
\end{aligned}
$$

Now, from (6.6), (6.5) and (6.7), one can choose first $M$ large enough and then $\rho_{j}$ small enough and $L$ large enough in order to conclude the proof.

\subsection{Normal density}

Here we treat instead the case of "normal" density $\rho_{j}$, namely we assume that $\rho_{j} \geqslant \rho_{0}$ for some constant $\rho_{0}$ independent of $L$. 
Proposition 6.3. - Assume USMT $(C, m, l)$. Then, for any $\varepsilon>0$ and any $0<\rho_{0}<$ $\frac{1}{2}$, there exists $C_{\varepsilon}$ such that for all $\rho_{0} \leqslant \rho_{j} \leqslant \frac{1}{2}$,

$$
v\left(f^{2}, G\right)^{2} \leqslant\left|\Lambda_{j}\right|\left[C_{\varepsilon}+\varepsilon \operatorname{Ent}_{v}\left(f^{2}\right)+L^{2} \mathcal{E}_{v}(f, f)\right] .
$$

Proof. - Fix $f$ with $v\left(f^{2}\right)=1$, together with $\varepsilon>0, \rho_{0}>0$ and $M$ large enough (how large will be specified later on).

As in Proposition 6.2 we define $t_{*}^{2}=\frac{1}{\left|\Lambda_{j}\right|}\left(1 \vee M \operatorname{Ent}_{v}\left(f^{2}\right)\right)$ where the number of particles $N_{j}$ has been replaced by the volume $\left|\Lambda_{j}\right|$ because we are assuming that $\rho_{j} \geqslant \rho_{0}$.

If $t_{*} \geqslant M$, then we can proceed as in (6.5) to get

$$
v\left(f^{2}, G\right)^{2} \leqslant \frac{k}{M}\left|\Lambda_{j}\right| \operatorname{Ent}_{v}\left(f^{2}\right)
$$

and the proof is finished provided that $M$ was taken large enough.

Let us now examine the much more complicate case of $t_{*} \leqslant M$.

We define $\left\{C_{\alpha}\right\}_{\alpha \in I}$ to be a collection of cubes of side $l_{0} \gg r$ multiple of $l$ inside $\Lambda_{j}$, such that

$\begin{aligned} \text { (i) } & \operatorname{dist}\left(C_{\alpha}, C_{\beta}\right) \geqslant l_{0}^{\frac{1}{2}} \quad \forall \alpha \neq \beta, \\ \text { (ii) } & \operatorname{dist}\left(C_{\alpha}, \partial \Lambda_{j}\right) \geqslant l_{0}^{\frac{1}{2}} \quad \forall \alpha, \\ \text { (iii) } & \left|\Lambda_{j} \backslash \bigcup_{\alpha} C_{\alpha}\right| \leqslant l_{0}^{-\frac{1}{2}}\left|\Lambda_{j}\right| .\end{aligned}$

Clearly such a construction is always possible. Define also $C_{\alpha}^{\mathrm{int}}=\left\{x \in C_{\alpha}: d\left(x, C_{\alpha}^{c}\right) \geqslant\right.$ $\left.l_{0}^{1 / 4}\right\}$ and $C^{\text {int }}=\bigcup_{\alpha} C_{\alpha}^{\text {int }}$.

Next we observe that, without loss of generality, we can replace $G$ by $G-\delta N_{\Lambda_{j}}$, $\delta$ being an arbitrary constant independent of $x$, because $N_{\Lambda_{j}}=N_{j}$ almost surely. Our choice of $\delta$ will be made later (see (6.16)) but we anticipate that it will be almost independent of $l_{0}$ and that under $\operatorname{USMT}(C, m, l)$, there exists a constant $\delta\left(C, m, l,\|g\|_{\infty}\right)$ such that $\delta \leqslant \delta\left(C, m, l,\|g\|_{\infty}\right)$ uniformly in $L$. Finally we set $g_{x}^{\delta}(\sigma):=g_{x}(\sigma)-\delta \sigma(x)$ and

$$
\begin{aligned}
G^{\mathrm{ext}} & =\sum_{x \in \Lambda_{j} \backslash C^{\mathrm{int}}}\left[g_{x}^{\delta}-\mu\left(g_{x}^{\delta}\right)\right], \\
G^{\mathrm{int}} & =\sum_{x \in C^{\mathrm{int}}}\left[g_{x}^{\delta}-\mu\left(g_{x}^{\delta}\right)\right] .
\end{aligned}
$$

Then we write

$$
\begin{aligned}
v\left(f^{2}, G^{\mathrm{ext}}\right)^{2} & \leqslant 2 v\left(f^{2} G^{\mathrm{ext}}\right)^{2}+2 v\left(G^{\mathrm{ext}}\right)^{2} \\
& \leqslant k\left|\Lambda_{j}\right|\left[1+\left(l_{0}^{-1} K+\frac{1}{M}\right) \operatorname{Ent}_{v}\left(f^{2}\right)\right]+k l_{0}^{-1} \\
& \leqslant\left|\Lambda_{j}\right|\left(k+\varepsilon \operatorname{Ent}_{v}\left(f^{2}\right)\right),
\end{aligned}
$$

where $K$ is some constant independent of $l_{0}$ but possibly dependent on $M$ and the last inequality holds if $M$ and $l_{0}$ are chosen appropriately. 
Above we have applied Lemma 6.1 with $t=t_{*}$ together with Proposition 5.4 point (i) to bound the first term and the equivalence of ensembles (see Proposition 3.2 point 1) together with (iii) above to bound the second one.

We now turn to the relevant term $v\left(f^{2}, G^{\text {int }}\right)^{2}$.

Let $\mathcal{F}_{0}$ be the $\sigma$-algebra generated by the random variables $\{\sigma(x)\}_{x \in \Lambda \backslash} \bigcup_{\alpha} C_{\alpha},\left\{N_{\alpha}\right\}_{\alpha \in I}$, where $N_{\alpha}(\sigma):=\sum_{x \in C_{\alpha}} \sigma(x)$. Then, by the formula for the conditional covariance, we get

$$
v\left(f^{2}, G_{\delta}^{\text {int }}\right)^{2} \leqslant 2 v\left(v\left(f^{2}, G^{\text {int }} \mid \mathcal{F}_{0}\right)\right)^{2}+2 v\left(f^{2}, v\left(G^{\text {int }} \mid \mathcal{F}_{0}\right)\right)^{2} .
$$

For simplicity let $v_{0}(\cdot):=v\left(\cdot \mid \mathcal{F}_{0}\right), f_{0}^{2}:=f^{2} / v_{0}\left(f^{2}\right)$ and $s_{*}^{2}:=\frac{M}{\left|\Lambda_{j}\right|} \operatorname{Ent}_{v_{0}}\left(f_{0}^{2}\right)$. Then the entropy inequality (Lemma 6.1) gives

$$
v_{0}\left(f_{0}^{2}, G^{\mathrm{int}}\right) \leqslant \frac{1}{s_{*}} \ln \left(v_{0}\left(\mathrm{e}^{s_{*}\left(G^{\mathrm{int}}-v_{0}\left(G^{\mathrm{int}}\right)\right)}\right)\right)+\frac{1}{s_{*}} \operatorname{Ent}_{v_{0}}\left(f_{0}^{2}\right) .
$$

Notice that $\nu_{0}$ is the product of standard canonical measures on each cube $C_{\alpha}$ with a certain number of particles and boundary conditions. Thus a simple Taylor expansion gives

$$
v_{0}\left(\mathrm{e}^{s_{*}\left(G^{\mathrm{int}}-\nu_{0}\left(G^{\mathrm{int}}\right)\right)}\right) \leqslant \mathrm{e}^{K s_{*}^{2}\left|\Lambda_{j}\right|},
$$

where $K$ now may depend on $l_{0}$. Thus

$$
v_{0}\left(f_{0}^{2}, G^{\mathrm{int}}\right) \leqslant K\left|\Lambda_{j}\right| s_{*}+\frac{1}{s_{*}} \operatorname{Ent}_{\nu_{0}} f_{0}^{2}
$$

and similarily for $G^{\text {int }}$ replaced by $-G^{\text {int }}$. The definition of $s_{*}$ yields

$$
v_{0}\left(f_{0}^{2}, G^{\text {int }}\right)^{2} \leqslant 2 K^{2}\left(M+\frac{1}{M}\right)\left|\Lambda_{j}\right| \operatorname{Ent}_{\nu_{0}}\left(f_{0}^{2}\right) .
$$

Finally, since $v_{0}$ is a product measure it certainly satisfies the logarithmic Sobolev inequality $\operatorname{Ent}_{v_{0}}\left(f_{0}^{2}\right) \leqslant C\left(l_{0}\right) \mathcal{E}_{v_{0}}\left(f_{0}, f_{0}\right)$, and we can conclude that

$$
v\left(v\left(f^{2}, G^{\text {int }} \mid \mathcal{F}_{0}\right)\right)^{2} \leqslant K^{\prime} \mathcal{E}_{v}(f, f)\left|\Lambda_{j}\right|,
$$

where $K^{\prime}=K^{\prime}\left(\|\Phi\|_{\infty},\|g\|_{\infty}, r, l_{0}, M\right)$.

The second term in the r.h.s. of (6.9) needs some more reductions. We recall first some definitions introduced in Section 5.

Given $\eta \in \Omega_{\Lambda_{j}}$ and $n \in\left[0, \ldots, l_{0}^{d}\right]$, write $\mu_{\alpha}^{\eta, n}(\cdot):=\mu_{C_{\alpha}}^{\eta, \lambda(\eta, n)}(\cdot)$ to denote the grand canonical Gibbs measure on $C_{\alpha}$ with boundary condition $\eta$ and constant chemical potential $\lambda(\eta, n)$ such that $\mu_{C_{\alpha}}^{\eta, \lambda(\eta, n)}\left(N_{C_{\alpha}}\right)=n$. We will use the standard notation $v_{C_{\alpha}, n}^{\eta}$ for the corresponding canonical Gibbs measure.

With this notation we define (see (5.7)),

$$
\xi_{\alpha}^{\delta}(\eta, n)=\sum_{x \in C_{\alpha}^{\mathrm{int}}} \mu_{\alpha}^{\eta, n}\left(g_{x}-\delta \sigma(x)\right) ; \quad \xi_{\alpha}^{\delta}(\eta):=\xi_{\alpha}^{\delta}\left(\eta, N_{C_{\alpha}}(\eta)\right)
$$




$$
g_{\alpha}^{\delta}(\eta, n)=\sum_{x \in C_{\alpha}^{\mathrm{int}}} v_{C_{\alpha}, n}^{\eta}\left(g_{x}-\delta \sigma(x)\right) ; \quad g_{\alpha}^{\delta}(\eta):=g_{\alpha}^{\delta}\left(\eta, N_{C_{\alpha}}(\eta)\right)
$$

and

$$
\begin{aligned}
G_{\nu}(\eta) & :=v\left(G^{\mathrm{int}} \mid \mathcal{F}_{0}\right)=\sum_{\alpha}\left[g_{\alpha}^{\delta}(\eta)-\mu\left(g_{\alpha}^{\delta}\right)\right], \\
G_{\mu}(\eta) & :=\sum_{\alpha}\left[\xi_{\alpha}^{\delta}(\eta)-\mu\left(\xi_{\alpha}^{\delta}\right)\right] .
\end{aligned}
$$

Then we write

$$
\begin{aligned}
& v\left(f^{2}, v\left(G^{\mathrm{int}} \mid \mathcal{F}_{0}\right)\right)^{2} \\
& \quad=v\left(f^{2}, G_{v}\right)^{2} \leqslant 4 v\left(f^{2}\left(G_{v}-G_{\mu}\right)\right)^{2}+4\left(v\left(G_{v}-G_{\mu}\right)\right)^{2}+2 v\left(f^{2}, G_{\mu}\right)^{2} .
\end{aligned}
$$

Let us examine the three terms separately.

Using Lemma 6.1 with $t=t_{*}$ combined with (ii) of Proposition 5.4, we can bound the first term by

$$
4 v\left(f^{2}\left(G_{v}-G_{\mu}\right)\right)^{2} \leqslant k\left|\Lambda_{j}\right|\left[1+\left(\frac{M A^{2}}{l_{0}^{2 d}}+\frac{1}{M}\right) \operatorname{Ent}_{v}\left(f^{2}\right)\right],
$$

where $A=A\left(C, m, l, M,\|g\|_{\infty}, \rho_{0}\right)$ is the constant appearing in Proposition 5.4.

Because of the equivalence of ensembles (see point (1) of Proposition 3.2) and the fact that $\mu\left(G_{\nu}-G_{\mu}\right)=0$

$$
4\left(v\left(G_{v}-G_{\mu}\right)\right)^{2} \leqslant k l_{0}^{2 d} .
$$

In conclusion, by a suitable choice of $M$ and $l_{0}$ we get

$$
4 v\left(f^{2}\left(G_{v}-G_{\mu}\right)\right)^{2}+4\left(v\left(G_{v}-G_{\mu}\right)\right)^{2} \leqslant\left|\Lambda_{j}\right|\left(C_{\varepsilon}+\varepsilon \text { Ent }_{v} f^{2}\right)
$$

for a suitable constant $C_{\varepsilon}$.

So, it remains to bound the third term in the r.h.s. of (6.11) $v\left(f^{2}, G_{\mu}\right)^{2}$. Here we are (unfortunately) forced to distinguish between two subcases.

(a) $t_{*} \leqslant M / l_{0}^{d}$.

We can appeal to Lemma 6.1 with $t=t_{*}$ combined with (iii), (iv) of Proposition 5.4 to get

$$
\begin{aligned}
v\left(f^{2}, G_{\mu}\right)^{2} & \leqslant 2 v\left(f^{2} G_{\mu}\right)^{2}+2\left|v\left(G_{\mu}\right)-\mu\left(G_{\mu}\right)\right|^{2} \\
& \leqslant k\left|\Lambda_{j}\right|\left[1+\left(\frac{M A^{2}}{l_{0}}+\frac{1}{M}\right) \operatorname{Ent}_{v}\left(f^{2}\right)\right]+k l_{0}^{2 d} \\
& \leqslant\left|\Lambda_{j}\right|\left(C_{\varepsilon}+\varepsilon \operatorname{Ent}_{v} f^{2}\right)
\end{aligned}
$$

for a suitable choice of $M, l_{0}$ and for all $L$ large enough.

(b) $M / l_{0}^{d} \leqslant t_{*} \leqslant M$.

In this case we can assume, without loss of generality, that $\left|\Lambda_{j}\right|$ is so large that $t_{*}^{2}=\frac{1}{\left|\Lambda_{j}\right|} M \operatorname{Ent}_{v}\left(f^{2}\right)$. 
We first need to simplify a bit the random variable $G_{\mu}$ by reducing it to a sum of variables each of which depends only on the number of particles in $C_{\alpha}^{\text {int }}$ and not also on the boundary condition outside $C_{\alpha}$.

Let $\xi_{\alpha}^{\delta, \underline{0}}(n):=\xi_{\alpha}^{\delta}(0, n)$ and let $G_{\mu}^{\underline{0}}(\eta)=\sum_{\alpha}\left[\xi_{\alpha}^{\delta, \underline{0}}\left(N_{C_{\alpha}}(\eta)\right)-\mu\left(\xi_{\alpha}^{\delta,} \underline{0}\right)\right]$. Notice that, by translation invariance inside $\Lambda_{j}, \xi_{\alpha}^{\delta, \underline{0}}(n)$ is independent of $\alpha$.

Using once more Proposition 3.2 we have $\left|\nu\left(G_{\mu}-G_{\mu}^{\frac{0}{\mu}}\right)\right| \leqslant k l_{0}^{d}$. Therefore

$$
v\left(f^{2}, G_{\mu}\right)^{2} \leqslant k\left[v\left(f^{2}\left(G_{\mu}-G_{\mu}^{0}\right)\right)^{2}+k l_{0}^{2 d}+v\left(f^{2}, G_{\mu}^{0}\right)^{2}\right] .
$$

Now, using Lemma 6.1 with $t=t_{*}$ together with Proposition 5.4 point (iii), we get

$$
v\left(f^{2}\left(G_{\mu}-G_{\mu}^{0}\right)\right)^{2} \leqslant k N_{j}\left(1+\frac{M A^{2}}{l_{0}^{2}}+\frac{1}{M} \operatorname{Ent}_{v} f^{2}\right) .
$$

In conclusion

$$
v\left(f^{2}, G_{\mu}\right)^{2} \leqslant\left|\Lambda_{j}\right|\left(C_{\varepsilon}+\varepsilon \operatorname{Ent}_{v} f^{2}\right)+k v\left(f^{2}, G_{\mu}^{0}\right)^{2}
$$

for suitably chosen $M$ and $l_{0}$.

We are left with the estimate of the term $v\left(f^{2}, G \frac{0}{\mu}\right)^{2}$. It is at this point that the substraction with the free parameter $\delta$ made at the beginning becomes important.

Let $\delta$ be such that for one (and therefore all) cube $C_{\alpha},\left.\frac{\mathrm{d}}{\mathrm{d} s} \xi_{\alpha}^{\delta,} \underline{0}(s)\right|_{s=\bar{n}}=0$ where $\bar{n}=\rho_{j}\left|C_{\alpha}\right|$. By definition, $\delta$ is independent of $\alpha$ and it is given by the following expression

$$
\delta=\frac{\mu_{C_{\alpha}}^{\underline{0}, \lambda(\underline{\underline{n}}, \bar{n})}\left(\sum_{x \in C_{\alpha}^{\mathrm{int}}} g_{x}, N_{C_{\alpha}}\right)}{\mu_{C_{\alpha}}^{\underline{0}, \lambda(\underline{n}, \bar{n})}\left(N_{C_{\alpha}^{\mathrm{int}}}, N_{C_{\alpha}}\right)}
$$

so that, thanks to the mixing hypothesis, $|\delta| \leqslant k$ uniformly in $L$.

Notice that with this choice,

$$
\left|\xi_{\alpha}^{\delta, 0}(n)-\xi_{\alpha}^{\delta, \underline{0}}(\bar{n})\right|=\left|\int_{\bar{n}}^{n} \mathrm{~d} s \int_{\bar{n}}^{s} \mathrm{~d} t \frac{\mathrm{d}^{2}}{\mathrm{~d} t^{2}} \xi_{\alpha}^{\delta}(0, t)\right| \leqslant k \frac{(n-\bar{n})^{2}}{\bar{n}}
$$

because of Lemma 3.1 (point (ii) of (2)). In turn (6.17) implies, in particular, that

$$
v\left(\left|\xi_{\alpha}^{\delta, \underline{0}}-\xi_{\alpha}^{\delta, \underline{0}}(\bar{n})\right|\right) \leqslant k \mu\left(\left|\xi_{\alpha}^{\delta, \underline{0}}-\xi_{\alpha}^{\delta, \underline{0}}(\bar{n})\right|\right) \leqslant k
$$

again because of the mixing condition.

LEMma 6.4. - Assume USMT $(C, m, l)$. Then, for any $\varepsilon>0$, there exists a constant $C_{\varepsilon}$ such that for all function $f$,

$$
v\left(f^{2}, G \frac{0}{\mu}\right)^{2} \leqslant\left|\Lambda_{j}\right|\left[C_{\varepsilon}+C_{\varepsilon} L^{2} \mathcal{E}_{v}(f, f)+\varepsilon \operatorname{Ent}_{v}\left(f^{2}\right)\right] .
$$


Proof. - First write $\left(n_{\alpha} \equiv N_{C_{\alpha}}(\eta)\right)$

$$
\begin{aligned}
v\left(f^{2}, G \frac{0}{\mu}\right)^{2} & \leqslant 2 v\left(f^{2} \sum_{\alpha \in I}\left[\xi_{\alpha}^{\delta, \underline{0}}\left(n_{\alpha}\right)-\xi_{\alpha}^{\delta, \underline{0}}(\bar{n})\right]\right)^{2}+2 v\left(\sum_{\alpha \in I}\left[\xi_{\alpha}^{\delta, \underline{0}}\left(n_{\alpha}\right)-\xi_{\alpha}^{\delta, \underline{0}}(\bar{n})\right]\right)^{2} \\
& \leqslant 2 v\left(f^{2} \sum_{\alpha \in I}\left[\xi_{\alpha}^{\delta,} \underline{0}\left(n_{\alpha}\right)-\xi_{\alpha}^{\delta,} \underline{0}(\bar{n})\right]\right)^{2}+k|I|^{2} \\
& \leqslant 2 v\left(f^{2} \sum_{\alpha \in I}\left[\xi_{\alpha}^{\delta, \underline{0}}\left(n_{\alpha}\right)-\xi_{\alpha}^{\delta,} \underline{0}(\bar{n})\right]\right)^{2}+k \frac{\left|\Lambda_{j}\right|}{M} \operatorname{Ent}_{v}\left(f^{2}\right)
\end{aligned}
$$

where we have used once more the equivalence of ensembles, Proposition 3.2 point (1), together with (6.17) and the fact that $t_{*} \geqslant \frac{M}{l_{0}^{d}}$.

We can thus focus our attention on the relevant term $v\left(f^{2} \sum_{\alpha \in I}\left[\xi_{\alpha}^{\delta,} \underline{0}\left(n_{\alpha}\right)-\xi_{\alpha}^{\delta,} \underline{0}(\bar{n})\right]\right)^{2}$. Thanks to (6.17) we can write

$$
v\left(f^{2} \sum_{\alpha \in I}\left[\xi_{\alpha}^{\delta,} \underline{0}\left(n_{\alpha}\right)-\xi_{\alpha}^{\delta,} \underline{0}(\bar{n})\right]\right)^{2} \leqslant k v\left(f^{2} \sum_{\alpha \in I} \frac{\left(n_{\alpha}-\bar{n}\right)^{2}}{\bar{n}}\right)^{2} .
$$

We now analyze the r.h.s. of (6.19). Let $\langle\cdot\rangle$ denote the average (normalized sum) over the cubes $\left\{C_{\beta}\right\}_{\beta \in I}$. Thanks to the conservation law we expect $\left\langle n_{\beta}\right\rangle$ to be quite close to $\bar{n}$. In fact, it is easy to check that $\left\langle n_{\beta}\right\rangle-\bar{n}=\frac{1}{|I|} \sum_{x \in \Lambda_{j} \backslash C}\left[\rho_{j}-\eta(x)\right]$. Notice that

$$
\sum_{y}\left\|\nabla_{y}\left[\sum_{x \in \Lambda_{j} \backslash C}\left(\rho_{j}-\eta(x)\right)\right]^{2}\right\|_{\infty}^{2} \leqslant k\left|\Lambda_{j} \backslash C\right|^{3} .
$$

Moreover, using the mixing condition together with the Poincaré inequality for $\mu$, we also have

$$
\begin{gathered}
\left|\mu\left(\sum_{x \in \Lambda_{j} \backslash C}\left[\rho_{j}-\eta(x)\right]\right)\right| \leqslant k \frac{\ln L}{L}\left|\Lambda_{j}\right|, \\
\operatorname{Var}_{\mu}\left(\sum_{x \in \Lambda_{j} \backslash C}\left[\rho_{j}-\eta(x)\right]\right) \leqslant k\left|\Lambda_{j} \backslash C\right|^{3} .
\end{gathered}
$$

Thus

$$
\begin{aligned}
v\left(f^{2} \sum_{\alpha} \frac{\left(\left\langle n_{\beta}\right\rangle-\bar{n}\right)^{2}}{\bar{n}}\right)^{2} & \leqslant k \frac{1}{\left|\Lambda_{j}\right|^{2}} v\left(f^{2}\left[\sum_{x \in \Lambda_{j} \backslash C}\left(\rho_{j}-\eta(x)\right)\right]^{2}\right)^{2} \\
& \leqslant k\left(\frac{\ln L}{L}\right)^{4}\left|\Lambda_{j}\right|^{2}+k \frac{1}{\left|\Lambda_{j}\right|^{2}}\left|\Lambda_{j} \backslash C\right|^{3} \operatorname{Ent}_{v}\left(f^{2}\right) \\
& \leqslant \varepsilon\left|\Lambda_{j}\right| \operatorname{Ent}_{v}\left(f^{2}\right)
\end{aligned}
$$

provided that $l_{0}$ and $L$ are large enough.

Above we have used Lemma 6.1 together with part (i) of Proposition 5.1 applied to the function $\hat{F}=F-\mu(F), F=\left[\sum_{x \in \Lambda_{j} \backslash C}\left(\rho_{j}-\eta(x)\right)\right]^{2}$, the bound $v\left(\mathrm{e}^{t \hat{F}}\right) \leqslant A \mu\left(\mathrm{e}^{t \hat{F}}\right)$ and the hypothesis $t_{*} \geqslant M / l_{0}^{d}$. 
Therefore we can safely add and subtract $\left\langle n_{\beta}\right\rangle$ inside the r.h.s. of (6.19) and confine ourselves to the estimate of $v\left(f^{2} \sum_{\alpha \in I} \frac{\left(n_{\alpha}-\left\langle n_{\beta}\right\rangle\right)^{2}}{\bar{n}}\right)^{2}$. We get

$$
\begin{aligned}
& v\left(f^{2} \sum_{\alpha \in I} \frac{\left(n_{\alpha}-\left\langle n_{\beta}\right\rangle\right)^{2}}{\bar{n}}\right)^{2} \\
& \quad \leqslant \frac{2}{|I|^{2}}\left[\sum_{\alpha, \beta} v\left(f^{2}, \frac{\left(n_{\alpha}-n_{\beta}\right)^{2}}{\bar{n}}\right)\right]^{2}+\frac{2}{|I|^{2}}\left[\sum_{\alpha, \beta} v\left(\frac{\left(n_{\alpha}-n_{\beta}\right)^{2}}{\bar{n}}\right)\right]^{2} .
\end{aligned}
$$

Since, by the strong mixing assumption and Proposition 3.3,

$$
v\left(\frac{\left(n_{\alpha}-n_{\beta}\right)^{2}}{\bar{n}}\right) \leqslant k \mu\left(\frac{\left(n_{\alpha}-n_{\beta}\right)^{2}}{\bar{n}}\right) \leqslant k,
$$

the second term in the r.h.s. of (6.21) is bounded by $k|I|^{2} \leqslant k \frac{\left|\Lambda_{j}\right|}{M} \operatorname{Ent}_{v}\left(f^{2}\right)$ because of the hypothesis $t_{*} \geqslant M / l_{0}^{d}$.

It now remains to bound the first term of (6.21). At this stage we cannot appeal to the same old argument based on the entropy inequality and we must proceed differently.

Following [19] we introduce $\mathcal{F}_{\alpha, \beta}$, the $\sigma$-algebra generated by the random variables $\{\sigma(x)\}_{x \in \Lambda \backslash\left(C_{\alpha} \cup C_{\beta}\right)}$, and we write

$$
\begin{aligned}
& \left|v\left(f^{2}, \frac{\left(n_{\alpha}-n_{\beta}\right)^{2}}{\bar{n}}\right)\right| \\
& \quad \leqslant\left|v\left(v\left(f^{2}, \frac{\left(n_{\alpha}-n_{\beta}\right)^{2}}{\bar{n}} \mid \mathcal{F}_{\alpha, \beta}\right)\right)\right|+\left|v\left(f^{2}, v\left(\frac{\left(n_{\alpha}-n_{\beta}\right)^{2}}{\bar{n}} \mid \mathcal{F}_{\alpha, \beta}\right)\right)\right| \\
& \quad \leqslant\left|v\left(v\left(f^{2}, \frac{\left(n_{\alpha}-n_{\beta}\right)^{2}}{\bar{n}} \mid \mathcal{F}_{\alpha, \beta}\right)\right)\right|+k,
\end{aligned}
$$

where we have used once more the equivalence of ensembles (Proposition 3.3), the mixing assumption and the fact that $n_{\alpha}\left(n_{\beta}\right)$ being the number of particles in $C_{\alpha}\left(C_{\beta}\right)$ is weakly sensitive to the boundary conditions outside $C_{\alpha}\left(C_{\beta}\right)$, to bound the second term.

Define now

$$
\mathcal{E}_{\alpha, \beta}(f, f)=\sum_{x, y \in C_{\alpha} \cup C_{\beta}} v\left(\left(\nabla_{x y} f\right)^{2} \mid \mathcal{F}_{\alpha, \beta}\right) .
$$

Obviously there exists a "spectral gap" constant $C\left(l_{0}\right)$ such that

$$
v\left(f, f \mid \mathcal{F}_{\alpha, \beta}\right) \leqslant C\left(l_{0}\right) \mathcal{E}_{\alpha, \beta}(f, f) \quad \forall f .
$$

Thus

$$
\begin{aligned}
\left|v\left(f^{2}, \frac{\left(n_{\alpha}-n_{\beta}\right)^{2}}{\bar{n}} \mid \mathcal{F}_{\alpha, \beta}\right)\right| & \leqslant 2\left\|\frac{\left(n_{\alpha}-n_{\beta}\right)^{2}}{\bar{n}}\right\|_{\infty}\left(v\left(f, f \mid \mathcal{F}_{\alpha, \beta}\right)\right)^{\frac{1}{2}}\left(v\left(f^{2} \mid \mathcal{F}_{\alpha, \beta}\right)\right)^{\frac{1}{2}} \\
& \leqslant l_{0}^{d} C\left(l_{0}\right)^{\frac{1}{2}}\left(\mathcal{E}_{\alpha, \beta}(f, f)\right)^{\frac{1}{2}}\left(v\left(f^{2} \mid \mathcal{F}_{\alpha, \beta}\right)\right)^{\frac{1}{2}} .
\end{aligned}
$$


Schwarz inequality yields

$$
v\left(\left|v\left(f^{2}, \frac{\left(n_{\alpha}-n_{\beta}\right)}{\bar{n}} \mid \mathcal{F}_{\alpha, \beta}\right)\right|\right) \leqslant C^{\prime}\left(l_{0}\right) v\left(\mathcal{E}_{\alpha, \beta}(f, f)\right)^{\frac{1}{2}} .
$$

Finally, from (6.22), we get that the first term in the r.h.s. of (6.21) is bounded from above by $\left(\right.$ define $\left.v_{\alpha, \beta}(\cdot):=v\left(\cdot \mid \mathcal{F}_{\alpha, \beta}\right)\right)$

$$
\begin{aligned}
& \frac{2}{|I|^{2}}\left[\sum_{\alpha, \beta} v\left(f^{2}, \frac{\left(n_{\alpha}-n_{\beta}\right)^{2}}{\bar{n}}\right)\right]^{2} \\
& \leqslant C^{\prime \prime}\left(l_{0}\right) \sum_{\alpha, \beta} v\left(\mathcal{E}_{\alpha, \beta}(f, f)\right)+k\left(\frac{\left|\Lambda_{j}\right|}{l_{0}^{d}}\right)^{2} \\
& \leqslant C^{\prime \prime}\left(l_{0}\right) \sum_{\alpha, \beta} v\left(\sum_{x, y \in C_{\alpha}} v_{\alpha, \beta}\left(\left[\nabla_{x} \nabla_{y} f\right]^{2}\right)+\sum_{x, y \in C_{\beta}} v_{\alpha, \beta}\left(\left[\nabla_{x} \nabla_{y} f\right]^{2}\right)\right. \\
& \left.\quad+\sum_{\substack{x \in C_{\alpha} \\
y \in C_{\beta}}} v_{\alpha, \beta}\left(\left[\nabla_{x} \nabla_{y} f\right]^{2}\right)\right)+\frac{k}{M}\left|\Lambda_{j}\right| \operatorname{Ent}_{v}\left(f^{2}\right) \\
& \leqslant C^{\prime \prime \prime}\left(l_{0}\right)\left|\Lambda_{j}\right|\left(l_{0}^{2}+L^{2}\right) \mathcal{E}(f, f)+\frac{k}{M}\left|\Lambda_{j}\right| \operatorname{Ent}_{v}\left(f^{2}\right)
\end{aligned}
$$

since $t_{*} \geqslant M / l_{0}^{d}$.

Above we have used once more the "path" bound $\sum_{x, y \in \Lambda_{j}} v\left(\nabla_{x y} f\right)^{2} \leqslant k L^{d+2} \mathcal{E}_{v}(f, f)$ given in (3.15). By choosing $M$ large enough we get the sought result also in this case.

\subsection{Applications}

Here we discuss an application of our results which is directly relevant for the proof of the diffusive scaling of the logarithmic Sobolev constant for the Kawasaki dynamics.

Fix $i, j \in\left\{1, \ldots, j_{\max }\right\}$ with $i \neq j$ and let $\rho=\frac{N_{j}+N_{j}}{\left|\Lambda_{j}\right|+\left|\Lambda_{j}\right|}$. Clearly $\frac{\delta_{0}}{2}\left(\rho_{i}+\rho_{j}\right) \leqslant \rho \leqslant$ $\rho_{i}+\rho_{j}$ where $\rho_{i}$ and $\rho_{j}$ are the densities in $\Lambda_{i}$ and $\Lambda_{j}$. Without loss of generality we assume that $\rho_{i} \leqslant \rho$ which implies $\rho_{j} \geqslant \rho_{i}$. Let also

$$
\begin{gathered}
g_{x}(\sigma)=\left[\mathrm{e}^{-\nabla_{x} H^{\tau}(\sigma)}-1\right] \sigma(x), \quad G:=\sum_{x \in \Lambda_{i}} g_{x}, \\
h_{x}(\sigma)=\left[\mathrm{e}^{-\nabla_{x} H^{\tau}(\sigma)}-1\right](1-\sigma(x)), \quad \mathcal{H}:=\sum_{z \in \Lambda_{j}} h_{z} .
\end{gathered}
$$

Notice that $g_{x}$ satisfies the hypotheses of Proposition 5.3 simply because $g_{x}=0$ if there are less than two particles (spins equal to 1 ) inside its support. Similarly $h_{z}=0$ if there is less than one particle inside its support. In particular $\|\mathcal{H}\|_{\infty} \leqslant k N_{j}$.

Proposition 6.5. - Assume condition $\operatorname{USMT}(C, m, l)$. Then for any $\varepsilon>0$ there exist $C_{\varepsilon}$ and $\rho_{0}$ such that for any $f$ with $v\left(f^{2}\right)=1$ 
(i) if $\rho \leqslant \rho_{0}$

$$
v\left(f^{2}, \sum_{\substack{x \in \Lambda_{i} \\ z \in \Lambda_{j}}} g_{x} h_{z}\right)^{2} \leqslant \rho|\Lambda|^{3}\left[C_{\varepsilon}+\varepsilon \operatorname{Ent}_{v}\left(f^{2}\right)\right]
$$

(ii) if $\rho>\rho_{0}$

$$
v\left(f^{2}, \sum_{\substack{x \in \Lambda_{i} \\ z \in \Lambda_{j}}} g_{x} h_{z}\right)^{2} \leqslant|\Lambda|^{3}\left[C_{\varepsilon}+C_{\varepsilon} L^{2} \mathcal{E}_{v}(f, f)+\varepsilon \operatorname{Ent}_{v}\left(f^{2}\right)\right]
$$

provided that $|\Lambda|$ is large enough.

Proof. - (i). Fix $\varepsilon>0$ and observe first that, thanks to Proposition 6.2, we can safely replace $\mathcal{H}$ and $G$ by $\mathcal{H}-\mu(\mathcal{H})$ and $G-\mu(G)$, respectively. The extra terms we get are in fact bounded by

$$
\begin{aligned}
|\mu(\mathcal{H})|^{2}\left(v\left(f^{2}, G\right)\right)^{2} & \leqslant\left|\Lambda_{j}\right|^{2} N_{i}\left(C_{\varepsilon}+\varepsilon \operatorname{Ent}_{v}\left(f^{2}\right)\right) \leqslant \rho|\Lambda|^{3}\left(C_{\varepsilon}+\varepsilon \operatorname{Ent}_{v}\left(f^{2}\right)\right) \\
|\mu(G)|^{2}\left(v\left(f^{2}, \mathcal{H}\right)\right)^{2} & \leqslant\left(\frac{\rho_{i}^{2}\left|\Lambda_{i}\right|+\rho_{i}\left|\partial^{+} \Lambda_{i}\right|}{\rho_{j}}\right)^{2} N_{j}\left(C_{\varepsilon}+\varepsilon \operatorname{Ent}_{v}\left(f^{2}\right)\right) \\
& \leqslant \rho|\Lambda|^{3}\left(C_{\varepsilon}+\varepsilon \operatorname{Ent}_{v}\left(f^{2}\right)\right)
\end{aligned}
$$

provided that $\rho$ is small enough. Above we have applied Proposition 6.2 to $G$ and $\rho_{j} \mathcal{H}$ respectively. Note that in the case $\rho_{i} \geqslant \rho$ which implies $\rho_{j} \leqslant \rho_{i}$ every thing is the same except that we apply Proposition 6.2 to $\rho \mathcal{H}$. From now on we will write $G$ for $G-\mu(G)$ and similarily for $\mathcal{H}$.

Next we write $\mathcal{H}=\mathcal{H}^{\text {in }}+\mathcal{H}^{\text {ext }}$ where $\mathcal{H}^{\text {in }}$ is the sum over those $z$ 's in $\Lambda_{j}$ such that $\Lambda_{h_{z}} \subset \Lambda_{j}$ and $\mathcal{H}^{\text {ext }}$ the rest. Similarily for $G$. Thus we have to bound four terms

$$
v\left(f^{2}, G^{\text {int }} \mathcal{H}^{\text {int }}\right)^{2}, \quad v\left(f^{2}, G^{\text {int }} \mathcal{H}^{\text {ext }}\right)^{2}, \quad v\left(f^{2}, G^{\text {ext }} \mathcal{H}^{\text {ext }}\right)^{2}, \quad v\left(f^{2}, G^{\text {ext }} \mathcal{H}^{\text {int }}\right)^{2} .
$$

All the mix terms can be treated similarily and therefore we will only analyze one of them e.g. $v\left(f^{2}, G^{\text {int }} \mathcal{H}^{\text {ext }}\right)^{2}$.

If we combine part (i) of Proposition 5.1 together with Lemma 6.1 we get

$$
\begin{aligned}
v\left(f^{2}, G^{\text {int }} \mathcal{H}^{\text {ext }}\right)^{2} & \leqslant k \inf _{t \geqslant 0}\left(\frac{k}{t}+t k c_{\mu}|\Lambda|^{2}\left|\partial^{+} \Lambda_{j}\right|+\frac{1}{t} \operatorname{Ent}_{v}\left(f^{2}\right)\right)^{2} \\
& \leqslant k|\Lambda|^{2}\left|\partial^{+} \Lambda_{j}\right| c_{\mu} \operatorname{Ent}_{v}\left(f^{2}\right) \\
& \leqslant k \rho|\Lambda|^{3} \operatorname{Ent}_{v}\left(f^{2}\right)
\end{aligned}
$$

for $|\Lambda|$ large enough, where $c_{\mu}$ denotes the logarithmic Sobolev constant of $\mu$ w.r.t. to the Heat Bath rates and we have used the fact (see the proof of Proposition 5.3) that $c_{\mu} \leqslant k \ln L$ uniformly in the (vector) chemical potential $\underline{\lambda}$.

We are left with the estimate of the "diagonal" term $\bar{v}\left(f^{2}, G^{\text {int }} \mathcal{H}^{\text {int }}\right)^{2}$. Let $\mathcal{F}_{i}=\mathcal{F}_{\Lambda_{i}^{c}}$. Then 


$$
\begin{aligned}
v\left(f^{2}, G^{\mathrm{int}} \mathcal{H}^{\mathrm{int}}\right)^{2} & \leqslant k N_{j}^{2} v\left(v\left(f^{2}, G \mid \mathcal{F}_{i}\right)^{2}\right)+2 v\left(f^{2}, \mathcal{H}^{\mathrm{int}} v\left(G^{\mathrm{int}} \mid \mathcal{F}_{i}\right)\right)^{2} \\
& \leqslant k N_{j}^{2} N_{i}\left(C_{\varepsilon}+\varepsilon \operatorname{Ent}_{v}\left(f^{2}\right)\right)+2 v\left(f^{2}, \mathcal{H}^{\mathrm{int}} v\left(G^{\mathrm{int}} \mid \mathcal{F}_{i}\right)\right)^{2}
\end{aligned}
$$

provided that $\rho$ is small enough. In order to estimate the last term in the r.h.s. of (6.24), we observe that, thanks to the strong mixing assumption, the fact that $\mu(G)=0$, and points (1) and (2) of Proposition 3.2 one has

$$
\left\|\nabla_{x} \mathcal{H}^{\text {int }} v\left(G^{\text {int }} \mid \mathcal{F}_{i}\right)\right\|_{\infty} \leqslant \begin{cases}k \rho_{i}\left|\partial^{+} \Lambda_{i}\right| & \text { if } \operatorname{dist}\left(x, \Lambda_{i}\right) \geqslant r \\ k N_{j} & \text { if } \operatorname{dist}\left(x, \partial^{+} \Lambda_{i}\right) \leqslant r\end{cases}
$$

In particular

$$
\sum_{x \in \Lambda_{j}}\left\|\nabla_{x} \mathcal{H}^{\mathrm{int}} v\left(G^{\mathrm{int}} \mid \mathcal{F}_{i}\right)\right\|_{\infty}^{2} \leqslant k \rho\left|\Lambda \| \partial^{+} \Lambda_{i}\right|^{2} .
$$

Thus, we can proceed as in (6.23) and get that also $v\left(f^{2}, \mathcal{H}^{\text {int }} v\left(G^{\text {int }} \mid \mathcal{F}_{i}\right)\right)^{2}$ is smaller than $k \rho|\Lambda|^{3}\left(C_{\varepsilon}+\varepsilon \operatorname{Ent}_{v}\left(f^{2}\right)\right)$.

To prove point (ii) the argument is unchanged with the only difference that now we must use Proposition 6.3 instead of Proposition 6.2.

\section{Acknowledgements}

C. Roberto would like to thank the Department of Mathematics of the University of Roma Tre for the hospitality and partial financial support during a three months visit.

\section{Appendix A. Logarithmic Sobolev inequality for one dimensional discrete random walks}

Let $\Omega=\left\{n_{\min }, n_{\min }+1, \ldots, n_{\max }\right\}$ be a finite subset of the integers and let $\gamma$ be a positive probability measure on $\Omega$. We present a result, based on Hardy type inequalities, to bound in a precise way the logarithmic Sobolev constant of $\gamma$ with respect to the Dirichlet form of discrete random walks reversible w.r.t. $\gamma$. After that we discuss a concrete example related to the distribution of the number of particles under a multicanonical measure (see Section 3.7).

PROPOSITION A.1. - For all functions $f$ on $\Omega$ we have

$$
\operatorname{Ent}_{\gamma}\left(f^{2}\right) \leqslant 20 B \sum_{n=n_{\min }+1}^{n_{\max }}(\gamma(n) \wedge \gamma(n-1))[f(n)-f(n-1)]^{2},
$$

where $B:=\inf _{i}\left[B_{+}(i) \vee B_{-}(i)\right]$, with

$$
\begin{aligned}
& B_{+}(i):=\sup _{n \geqslant i+1}\left(\sum_{k=n}^{n_{\max }} \gamma(k)\right) \ln \left(\frac{1}{\sum_{k=n}^{n_{\max }} \gamma(k)}\right)\left(\sum_{k=i+1}^{n} \frac{1}{\gamma(k) \wedge \gamma(k-1)}\right), \\
& B_{-}(i):=\sup _{n \leqslant i-1}\left(\sum_{k=n_{\min }}^{n} \gamma(k)\right) \ln \left(\frac{1}{\sum_{k=n_{\min }}^{n} \gamma(k)}\right)\left(\sum_{k=n}^{i-1} \frac{1}{\gamma(k) \wedge \gamma(k+1)}\right) .
\end{aligned}
$$


Proof. - The proof is a straightforward application of Hardy type inequalities (see [17, Section 3]). Indeed, it's enough to consider the birth and death continuous time Markov chain (Metropolis chain) with transition rates

$$
c(n, j)= \begin{cases}\frac{\gamma(j)}{\gamma(n)} \wedge \frac{1}{2} & \text { if } j=n \pm 1, \\ 0 & \text { otherwise }\end{cases}
$$

We now define a convexity assumption for the probability measure under consideration that will allow us to bound rather sharply the constant $B$ described above.

DEFINITION OF CONDITION $\operatorname{CONV}(c, \bar{n})$. - We say that $\gamma$ satisfies the convexity hypothesis with parameters $c>0$ and $\bar{n} \in \Omega$, in what follows denoted by $\operatorname{CONV}(c, \bar{n})$, if $c^{-1} \bar{n} \leqslant n_{\max }-\bar{n} \leqslant c \bar{n}$ and similarily for $\bar{n}-n_{\min }$,

$$
\begin{gathered}
\frac{\gamma(n+1)}{\gamma(n)} \leqslant c \mathrm{e}^{-\frac{(n-\bar{n})}{c \bar{n}}} \quad \text { for all } n \geqslant \bar{n}, \\
\frac{\gamma(n-1)}{\gamma(n)} \leqslant c \mathrm{e}^{-\frac{(\bar{n}-n)}{c \bar{n}}} \quad \text { for all } n \leqslant \bar{n}, \\
\frac{1}{c \sqrt{\bar{n}}} \mathrm{e}^{-\frac{c(\bar{n}-n)^{2}}{\bar{n}}} \leqslant \gamma(n) \leqslant \frac{c}{\sqrt{\bar{n}}} \mathrm{e}^{-\frac{(\bar{n}-n)^{2}}{c \bar{n}}} \quad \text { for all } n \in \Omega .
\end{gathered}
$$

Remark. - Since $\gamma$ is assumed to be a positive probability measure, we can always write it in the Gibbsian form $\gamma(n)=Z^{-1} \exp (-H(n))$. Then, if $H$ is strictly convex with "second derivative" bounded from below and from above by $c^{-1}$ and $c$ respectively, and the minimum in $\bar{n}$, i.e.

$$
\begin{aligned}
& \frac{1}{c \bar{n}} \leqslant H(n+2)-2 H(n+1)+H(n) \leqslant \frac{c}{\bar{n}} \quad \forall n \in \Omega, \\
& H(\bar{n}+1)=H(\bar{n}),
\end{aligned}
$$

then, if $\left(c, \bar{n}, n_{\max }, n_{\min }\right)$ are in the right proportion described in $\operatorname{CONV}(c, \bar{n}), \gamma$ satisfies condition $\operatorname{CONV}(c, \bar{n})$.

Our aim now is to prove that under $\operatorname{CONV}(c, \bar{n})$ the measure $\gamma$ satisfies a logarithmic Sobolev inequality with optimal constant proportional (depending on $c$ ) to $\bar{n}$ (see Proposition A.5 below). For this purpose, let us first discuss some simple lemma that deal with the different terms appearing in the constants $B_{+}(i)$ and $B_{-}(i)$ of Proposition A.1. Since obviously $B \leqslant B_{+}(\bar{n}) \vee B_{-}(\bar{n})$ we can restrict us to the case $i=\bar{n}$.

Lemma A.2. - Let $\gamma$ satisfies $\operatorname{CONV}(c, \bar{n})$. Then

$$
\begin{gathered}
\sum_{k=n}^{n_{\max }} \gamma(k) \leqslant C \frac{\bar{n}}{n-\bar{n}} \gamma(n) \quad \text { for all } n \geqslant \bar{n}+1, \\
\sum_{k=n_{\min }}^{n} \gamma(k) \leqslant C \frac{\bar{n}}{\bar{n}-n} \gamma(n) \text { for all } n \leqslant \bar{n}-1,
\end{gathered}
$$

for a suitable numerical constant $C$ depending only on $c$ and not on $\bar{n}$. 
Proof. - Fix $n \geqslant \bar{n}+1$. By using the definition of $\operatorname{CONV}(c, \bar{n})$ together with a simple telescopic argument we get

$$
\begin{aligned}
\sum_{k=n}^{n_{\max }} \frac{\gamma(k)}{\gamma(n)} & =\sum_{k=n}^{n_{\max }} \prod_{j=n}^{k-1} \frac{\gamma(j+1)}{\gamma(j)} \leqslant c \sum_{k=n}^{n_{\max }} \prod_{j=n}^{k-1} \exp \left(-\frac{(j-\bar{n})}{c \bar{n}}\right) \\
& \leqslant c \sum_{k \geqslant n} \exp \left(-\frac{(k-n)(n-\bar{n})}{2 c \bar{n}}\right)=c \frac{1}{1-\exp \left(-\frac{(n-\bar{n})}{2 c \bar{n}}\right)} .
\end{aligned}
$$

But, $(n-\bar{n}) / \bar{n} \leqslant\left(n_{\max }-\bar{n}\right) / \bar{n} \leqslant c$ and therefore

$$
\left(1-\exp \left(-\frac{(n-\bar{n})}{2 c \bar{n}}\right)\right)^{-1} \leqslant C \frac{\bar{n}}{n-\bar{n}}
$$

for some constant $C$ depending only on $c$. A similar computation gives the corresponding result for $n \leqslant \bar{n}-1$. This achieves the proof.

Lemma A.3. - Let $\gamma$ satisfies $\operatorname{CONV}(c, \bar{n})$. Then

$$
\begin{gathered}
\sum_{k=\bar{n}+1}^{n} \frac{1}{\gamma(k)} \leqslant C \frac{\bar{n}}{n-\bar{n}} \frac{1}{\gamma(n)} \text { for all } n \geqslant \bar{n}+1, \\
\sum_{k=n}^{\bar{n}-1} \frac{1}{\gamma(k)} \leqslant C \frac{\bar{n}}{\bar{n}-n} \frac{1}{\gamma(n)} \text { for all } n \leqslant \bar{n}-1,
\end{gathered}
$$

for a suitable numerical constant $C$ depending only on $c$.

Proof. - The proof is practically the same as that of Lemma A.2 and it is omitted.

LemmA A.4. - Let $\gamma$ satisfies $\operatorname{CONV}(c, \bar{n})$. Then

$$
\begin{array}{r}
\ln \left(\frac{1}{\sum_{k=n}^{n_{\max }} \gamma(k)}\right) \leqslant C\left(1+\frac{(\bar{n}-n)^{2}}{\bar{n}}\right) \quad \text { for all } n \geqslant \bar{n}+\sqrt{\bar{n}}, \\
\ln \left(\frac{1}{\sum_{k=n_{\min }}^{n} \gamma(k)}\right) \leqslant C\left(1+\frac{(\bar{n}-n)^{2}}{\bar{n}}\right) \text { for all } n \leqslant \bar{n}-\sqrt{\bar{n}},
\end{array}
$$

where $C$ is a suitable numerical constant depending only on $c$.

Proof. - If $\bar{n}$ is smaller than some large constant depending on $c$ there is nothing to be proved. Therefore we can assume that $\bar{n}$ is large enough and we consider two cases.

In the first one, we suppose $\bar{n}+\sqrt{\bar{n}} \leqslant n \leqslant n_{\max }-\sqrt{\bar{n}}$. By using the definition of $\operatorname{CONV}(c, \bar{n})$ we get

$$
\begin{aligned}
\sum_{k=n}^{n_{\max }} \gamma(k) & \geqslant \frac{1}{c} \sum_{k=n}^{n_{\max }} \frac{1}{\sqrt{\bar{n}}} \exp \left(-\frac{c}{\bar{n}}(\bar{n}-k)^{2}\right) \geqslant \frac{1}{c} \sum_{k=n}^{n+\sqrt{\bar{n}}} \frac{1}{\sqrt{\bar{n}}} \exp \left(-\frac{c}{\bar{n}}(\bar{n}-k)^{2}\right) \\
& \geqslant \frac{1}{c} \sqrt{\bar{n}} \frac{1}{\sqrt{\bar{n}}} \exp \left(-\frac{c}{\bar{n}}(n+\sqrt{\bar{n}}-\bar{n})^{2}\right) \geqslant \frac{1}{c} \exp \left(-\frac{4 c}{\bar{n}}(n-\bar{n})^{2}\right),
\end{aligned}
$$

where, in the last inequality, we have used the fact that $(n+\sqrt{\bar{n}}-\bar{n})^{2} \leqslant 4(n-\bar{n})^{2}$ for all $n \geqslant \bar{n}+\sqrt{\bar{n}}$. 
In the second case, we suppose $n \geqslant n_{\max }-\sqrt{\bar{n}}$. An easy computation gives

$$
\begin{aligned}
\sum_{k=n}^{n_{\max }} \gamma(k) & \geqslant \frac{1}{c} \sum_{k=n}^{n_{\max }} \frac{1}{\sqrt{\bar{n}}} \exp \left(-\frac{c}{\bar{n}}(\bar{n}-k)^{2}\right) \geqslant \frac{1}{c} \frac{1}{\sqrt{\bar{n}}} \exp \left(-\frac{c}{\bar{n}}(\bar{n}-n)^{2}\right) \\
& \geqslant C^{-1} \exp \left(-\frac{C}{\bar{n}}(\bar{n}-n)^{2}\right)
\end{aligned}
$$

for some constant $C$ depending only on $c$. Finally the first statement of the lemma follows at once from (A.4) and (A.5) by taking the logarithm. Similarly one proceeds for the proof of the second part of the lemma.

We can now state the main result of the appendix.

Proposition A.5. - Let $\gamma$ satisfies $\operatorname{CONV}(c, \bar{n})$. Then there exists a constant $C$ depending only on $c$ such that for all real functions $f$ on $\Omega$

$$
\operatorname{Ent}_{\gamma}(f) \leqslant C \bar{n} \sum_{n=n_{\min }+1}^{n_{\max }}(\gamma(n) \wedge \gamma(n-1))[f(n)-f(n-1)]^{2} .
$$

Proof. - Thanks to Proposition A.1 it is enough to bound from above the constant $B$ or, what is enough $B_{+}(\bar{n}) \vee B_{-}(\bar{n})$. First notice that by symmetry we can only consider one of them e.g.

$$
B_{+}(\bar{n})=\sup _{n \geqslant \bar{n}+1}\left(\sum_{k=n}^{n_{\max }} \gamma(k)\right) \ln \left(\frac{1}{\sum_{k=n}^{n_{\max }} \gamma(k)}\right)\left(\sum_{k=\bar{n}+1}^{n} \frac{1}{\gamma(k) \wedge \gamma(k+1)}\right) .
$$

Let us consider two cases.

In the first one, we suppose $\bar{n}+1 \leqslant n \leqslant \bar{n}+\sqrt{\bar{n}}$. Then, because $\sum_{k=n}^{n_{\max }} \gamma(k) \leqslant 1$ and $x \ln (1 / x) \leqslant 1$ for all $x \in[0,1]$,

$$
\left(\sum_{k=n}^{n_{\max }} \gamma(k)\right) \ln \left(\frac{1}{\sum_{k=n}^{n_{\max }} \gamma(k)}\right) \leqslant 1
$$

Moreover, by (A.2)

$$
\sum_{k=\bar{n}+1}^{n} \frac{1}{\gamma(k)} \leqslant c \sum_{k=\bar{n}+1}^{n} \frac{1}{\sqrt{\bar{n}}} \exp \left(\frac{c}{\bar{n}}(\bar{n}-k)^{2}\right) \leqslant c \sqrt{\bar{n}} \frac{1}{\sqrt{\bar{n}}} \exp \left(\frac{C}{\bar{n}}(\bar{n}-n+1)^{2}\right) \leqslant C
$$

for some constant $C$ depending only on $c$.

In the second case, $n \geqslant \bar{n}+\sqrt{\bar{n}}$, we apply Lemmas A.2, A.3 and A.4 to get

$$
\begin{aligned}
\sum_{k=n}^{n_{\max }} \gamma(k) \ln \left(\frac{1}{\sum_{k=n}^{n_{\max }} \gamma(k)}\right) \sum_{k=\bar{n}+1}^{n} \frac{1}{\gamma(k)} \leqslant C \frac{\bar{n}}{n-\bar{n}}\left(1+\frac{(\bar{n}-n)^{2}}{\bar{n}}\right) \frac{\bar{n}}{n-\bar{n}} \\
\leqslant C \bar{n} .
\end{aligned}
$$

This achieves the proof. 


\section{REFERENCES}

[1] Albanese C., A Goldstone mode in the Kawasaki-Ising model, J. Stat. Phys. 77 (1/2) (1994) 77-87.

[2] Ané C. et al., Sur les inégalités de Sobolev logarithmiques, Panoramas et Synthèses 10 (2001), S.M.F.

[3] Bertini L., Cancrini N., Cesi F., The spectral gap for a Glauber-type dynamics in a continuous gas, Preprint n. 00-249 on http://rene.ma.utexas.edu/mp_arc.

[4] Bertini L., Cirillo E.N.M., Olivieri E., Renormalization-group transformations under strong mixing conditions: Gibbsianness and convergence of renormalized interactions, J. Stat. Phys. 97 (1999) 831-915.

[5] Bertini L., Zegarlinski B., Coercive inequalities for Gibbs measures, J. Funct. Anal. 162 (1999) 257-289.

[6] Bertini L., Zegarlinski B., Coercive inequalities for Kawasaki dynamics. The product case, Markov Proc. Related Fields 5 (1999) 125-162.

[7] Cancrini N., Cesi F., Martinelli F., Kawasaki dynamics at low temperature, J. Stat. Phys. 95 (1/2) (1999) 219-275.

[8] Cesi F., Quasi-factorization of the entropy and logarithmic Sobolev inequalities for Gibbs random fields, Probab. Theory Related Fields (2001). To appear.

[9] Cancrini N., Martinelli F., Comparison of finite volume canonical and grand canonical Gibbs measures under a mixing condition, Markov Proc. Related Fields 6 (2000) 1-49.

[10] Cancrini N., Martinelli F., On the spectral gap of Kawasaki dynamics under a mixing condition revisited, J. Math. Phys. 41 (2000) 1391-1423.

[11] Cancrini N., Martinelli F., Diffusive scaling of the spectral gap for the dilute Ising lattice gas under the percolation threshold, Probab. Theory Related Fields (2001). To appear.

[12] Diaconis P., Saloff-Coste L., Logarithmic Sobolev inequality for finite Markov chains, Ann. Appl. Probab. 6 (3) (1996) 695-750.

[13] Georgii H.O., Gibbs Measures and Phase Transitions, De Gruyter Series in Mathematics, Vol. 9, Walter de Gruyter, Berlin, 1988.

[14] Lu S.T., Yau H.-T., Spectral gap and logarithmic Sobolev inequality for Kawasaki and Glauber dynamics, Comm. Math. Phys. 156 (1993) 399-433.

[15] Lee T.-Y.T., Yau H.-T., Logarithmic Sobolev inequality for some models of random walks, Ann. Probab. 26 (4) (1998) 1855-1873.

[16] Martinelli F., Lectures on Glauber dynamics for discrete spin models, Proceedings of the Saint Flour Summer School in Probability Theory, Lecture Notes in Math., Vol. 1717, 1997.

[17] Miclo L., An example of application of discrete Hardy's inequalities, Markov Process. Related Fields 5 (1999) 319-330.

[18] Varadhan S.R.S., Yau H.-T., Diffusive limit of lattice gas with mixing conditions, Asian J. Math. 1 (4) (1997) 623-678.

[19] Yau H.-T., Logarithmic Sobolev inequality for lattice gases with mixing conditions, Comm. Math. Phys. 181 (1996) 367-408.

[20] Yau H.-T., Logarithmic Sobolev inequality for generalized simple exclusion processes, Probab. Theory Related Fields 109 (4) (1997) 507-538. 